\title{
Optical and electrostatic potential investigations of electrical breakdown phenomena in a low pressure gas discharge lamp
}

\author{
M F Gendre ${ }^{1}$, M Haverlag ${ }^{1,2}$ and G W M Kroesen ${ }^{2}$ \\ ${ }^{1}$ LighLabs, Philips Lighting, Mathildelaan 1, 5611 BD, Eindhoven, the Netherlands \\ ${ }^{2}$ Eindhoven University of Technology, Department of Applied Physics, PO box 513, 5600 MB Eindhoven, the \\ Netherlands \\ E-mail: maxime.gendre@philips.com
}

\begin{abstract}
The ignition phase is a critical stage in the operation of gas discharge lamps where the neutral gas enclosed between the electrodes undergoes a transformation from the dielectric state to a conducting phase, eventually enabling the production of light. The phenomena occurring during this phase transition are not fully understood and the related experimental studies are often limited to local optical measurements in environments prone to influencing these transient phenomena. In this work unipolar ignition phenomena at sub-kilovolt levels are investigated in a 3 Torr argon discharge tube. The lamp is placed in a highly controlled environment so as to prevent any bias on the measurements. A fast intensified CCD camera and a specially-designed novel electrostatic probe are used simultaneously so as to provide with a broad array of measured and computed parameters which are displayed in space-time diagrams for cross comparisons. Experiments show that three distinct phases exist during successful ignitions: Upon the application of voltage a first ionization wave starts from the active electrode and propagates in the neutral gas toward the opposite electrode. A local front of high axial $\mathrm{E}$ field strength is associated with this process and causes a local ionisation to occur, leading to the electrostatic charging of the lamp. Next, a second wave propagates from the ground electrode back toward the active electrode with a higher velocity, and in this process leads to a partial discharging of the lamp. This return stroke draws a homogeneous plasma column which eventually bridges both electrodes at the end of the wave propagation. At this point both electrode sheaths are formed and the common features of a glow discharge are observed. The third phase is an increase of the light intensity of the plasma column until the lamp reaches a steady state operation. Failed ignitions present only the first phase where the first wave starts its propagation but extinguishes in the lamp, leading to a charge memory effect. It is found that the full propagation of this first wave is a requirement for a successful lamp ignition. Differences in the properties of the waves were observed depending on the voltage polarity, and it was estimated that a photoelectric effect at the wall is the most likely source of electron for the ionisation wave of positive polarity. Finally a simple model of the first ionization wave is developed and used to analyse the fundamental differences between processes occurring at negative and at positive polarity. From this study three conditions are developed for the successful unipolar ignition of lamps and the relations between them are derived.
\end{abstract}




\section{Introduction}

The invention of the electric pile in 1880 by A. Volta enabled for the first time the continuous supply of an electric current, which started the era of electric lighting [1]. Electric discharge tubes became commercially significant only in 1901, with the introduction of the low-pressure mercury lamp (P. Cooper-Hewitt) [2], and of the neon tubes in 1910 (G. Claude) [3] which performed better and longer than incandescent lamps. The process of initiating the discharge was very crude in these early lamps; it relied on contact breaking from liquid mercury [4], on high-frequency Tesla coils and on transformers with high open-circuit voltages [5], resulting in complex systems [6]. The development of externallyheated oxide-coated cathodes by A. Wehnelt in 1904 and the introduction of modern fluorescent tubes in 1938 (General Electric, [7]) led to discharge lamps easier to ignite and operate [8]. The technology gradually improved in time, resulting in the energy-efficient compact fluorescent lamp (CFL) aimed at replacing filament lamps in their sockets (Philips, 1981 [9]). In 2005 these fluorescent lamps accounted for more than half of the total amount of generated light (133 Petalumen-hours) [10], which is a clear indication of their market dominance. The efficiency and cost of these systems [11] are therefore of importance, and a further improvement of this technology can be achieved via the better understanding of the ignition processes since high-voltage circuits are still relied on for starting.

The ignition phase occurs at the moment when the power supply is switched on and a potential difference is applied across the electrodes of the discharge tube. At that moment the gaseous fill of the lamp passes from the dielectric (insulating) state to an electrically-conducting phase. Although the Townsend mechanism of electron avalanche is well known [12] and is often used to understand the phenomena associated with ignition, its idealized description cannot be applied to the geometry of most discharge lamps, and of fluorescent tubes (and CFLs) in particular. In these lamps the length between the electrodes is much larger than the tube diameter; the dielectric walls therefore play a crucial role in the mechanisms leading to electrical conduction [13,14].

Up to now the ignition processes in low-pressure discharge lamps have been only observed locally by means of capacitive probes [15,16,17], Langmuir probes [17,18,19] and/or fiber-coupled photomultipliers $[15,17,20]$. The global properties and behavior of ignition phenomena were therefore inferred from these local measurements. Although the data retrieved from electric diagnostic tools can provide more information than optical measurements, their use most often leads to strong disturbances on the measured phenomena, especially in the case of Langmuir probes. The underlying reason for this problem is the fact that these tools are not suitable for the analysis of a transient phenomenon like gas breakdown processes, which can be disturbed by any small change of their electrostatic environment. Horstman et al. [16] recognized this problem and merged their capacitive probe with a cylindrical Faraday cage to suppress any local fringing field which could bias the measurements. However, the need for the highest spatial resolution of their measurements requires than the Faraday cage fits the lamp tightly, leading to an electrostatic configuration which is very different from the situation found in practical lighting systems.

The optical analysis of the light emitted from igniting lamps is intrinsically non-invasive in nature, and such diagnostics are therefore less likely to influence the lamp during this phase. However, the amount of information retrieved is limited to the light intensity, position and speed of observed features. Moreover, the majority of optical diagnostics used for this investigation are only resolved with a few measurement points ( 8 or less) along the lamp axis, and it is upon this limited information that many conclusions and theories are drawn about the mechanisms underlying the ignition processes.

Nevertheless, experiments using these diagnostic tools were critically useful for the first observations and analysis of breakdown processes in long discharge lamps. It is agreed from most observations $[15,16,17,18]$ that one or more ionization waves propagate between the electrodes before 
these are fully bridged by a glow discharge. The first of these waves is found to be initiated around the electrode whose local electric field is the strongest. This local field leads to a Townsend-like breakdown between this electrode and the nearest section of the lamp wall, which results in its electrostatic charging up to the potential of the adjacent electrode [15]. The local breakdown wave front starts to move forward (the pre-breakdown wave) as the region of strongest $\mathrm{E}$ field is displaced by wall charges, which cause further Townsend-like ionization to occur ahead $[15,16]$. The displaced ionization region creates free charges ahead of the front that will progressively extend the conduction path from one electrode to the opposite one. Once the ionization wave has reached the opposite electrode a second wave front develops (the return stroke) and moves back toward the electrode where the first wave started. It has been suggested that this propagating wave partially discharges the wall [16]. This mechanism of surface charge removal is not precisely known but Horstman et al. describe a discharge of the wall surface via a current through the plasma without detailing the mechanisms of neutralization involved. Their observation showed that the speed of this return stroke is much higher than that of the pre-breakdown wave front, but did not provide any explanation for this characteristic.

Until now, all observations of these waves have been done only in an indirect and localized manner, which does not permit a global lamp-wide visualization of the phenomenon evolution over time. One exception to this is the study from von Busch et al. [21] who used a fast intensified CCD camera to map the optical emission in capillary neon lamps ignited at $3 \mathrm{kV}$. Most of these experiments were performed with voltage pulses in the $\mathrm{kV}$ range or higher, leading to conditions remote from those found in practical fluorescent tubes and systems during ignition. Moreover, in most studies little care was taken toward the protection of the discharge tube from external electrostatic and atmospheric influences. In the few cases that a Faraday cage was used, the electric field strength present near the electrodes was much greater than usually present in real-life situations.

The lamp used in the present work is a low-pressure discharge tube whose structure is similar to those used in CFLs, but has a linear geometry, is un-coated and is filled with argon at 4 mbar. The lamp was ignited with unipolar voltage pulses of positive and negative polarity whose amplitude ranges from 100 to $800 \mathrm{~V}$. The experimental parameters were chosen so as to provide the simplest case of study enabling the investigation of the fundamental processes occurring during breakdown. Such ideal situation was modeled by Brok et al. [22,23] and provides with some critical insights on the microscopic processes occurring inside the lamp. However, the many simplifications made lead to discrepancies with experiments; the nature of the model does not enable the study of positive ignitions and one cannot derive scaling laws for understanding the relative importance of lamp parameters. Such problems are also encountered with other numerical models like that from Horstman et al. [16].

The goals of this study is therefore to provide facts, and then develop an analytical model of the macroscopic breakdown processes backed by the experimental data provided via the combined use of two diagnostic tools under a tightly controlled lamp environment. The first diagnostic is a fast intensified CCD camera which was used for the mapping of light emission associated with the propagating ionization waves. The second instrument, emphasized in this article, is a novel electrostatic probe which was specifically designed to have the smallest impact possible on the breakdown phenomena so as to limit the biasing of experimental results. Several useful parameters such as the electric field, the charge density and the current fluxes are computed from the data provided by the electrostatic probe. From these results we assess the effect on breakdown kinetics of the amplitude of the applied voltage and of signal polarity. The experiments are carefully controlled both on the aspects of measurement quality and of electrostatic and atmospheric environment of the lamp. The first part of this article deals with details related to the lamps under investigation, the experimental set-up and the novel electrostatic probe. The next two sections provide the optical and 
electrostatic results related to ignition at negative and positive potentials. The time evolutions of the wave position, velocity, E field strength, and potential are given as well as the evolution of the lamp total net charge and displacement current. The fourth and final part presents the analytical model of the first ionization wave used to better understand the investigated phenomena and to find the fundamental differences in the breakdown processes occurring at negative and at positive polarity.

\section{Set-up and experimental procedures}

\subsection{The lamp under investigation}

The lamp used in the experiments has only the tube diameter, the wall thickness and the electrode construction that are comparable to normal CFLs. Other characteristics, however, are different; first of all the lamp is filled with argon at 3 Torr (4 mbar), which normally is the buffer of standard CFLs, but no mercury is used in the present study so as to enable the observation of breakdown processes without Penning reaction $[14,24]$. Standard CFLs usually have several folded compact fluorescent tubes linked together through bridges. In the present study a simpler lamp design with a straight tube was used in order to have a simpler geometry to investigate. The diagram of figure 1 shows the geometry and dimensions of the lamp, made of a soft soda-lime silicate glass (Philips type 360) and optically transparent in the spectral range from $340 \mathrm{~nm}$ to $1200 \mathrm{~nm}$.

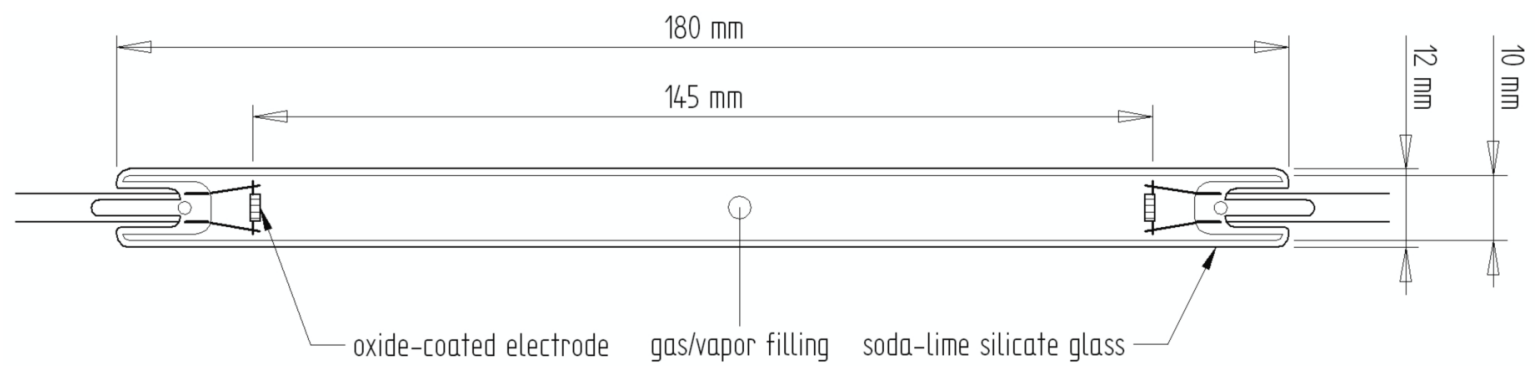

Figure 1: Structure of the linear lamp used for the experiments. The lamp is made of soft soda-lime silicate glass and has tungsten electrodes of the triple-wound type coated with oxides of barium, strontium and calcium.

Like their CFL counterparts these experimental lamps were provided with standard electrodes made of a multi-coiled tungsten filament coated with a mixture of barium-calcium-strontium oxides, which promote electron emission [14]. These electrodes are mounted to lead wires which allow their connection to an external current source for the setting and control of their temperature.

The lamp was processed prior to the first breakdown experiments so as to keep the level of impurities as low as possible. It is found that the most important contaminant resulting from lamp manufacture is nitrogen. This molecular gas has a negative impact on argon-mercury and on argon discharges in general [7] and on ignition in particular (i.e. it leads to higher breakdown voltages and to slower breakdown kinetics) and its clean-up was done by operating the lamp at between 400 and 500 $\mathrm{mA}$ for several hours until the measured effects of nitrogen are no longer observed. The high energy electrons from the electrical discharge are responsible for breaking up the $\mathrm{N}_{2}$ molecule. This dissociation reaction releases highly reactive nitrogen atoms which will then react with the electrode and the glass materials [25], which then act as efficient getters. In the trade it is customary to operate lamps for 100 hours at nominal regime in order to properly stabilize the electrode work function and the electrical characteristics of the lamp [26]. We observed that operating the lamp at high current (our 
electrodes are normally designed for discharges at $315 \mathrm{~mA}$ ) leads to a stabilization after less than 10 hours (less than 5 hours in most cases). A shorter processing time is important to limit the amount of metallic barium released from the oxide electrodes and deposited onto the lamp wall. The influence of these alterations to the lamp structure on breakdown processes are not known or understood, so to avoid any potential complications and unforeseen effects the extent of these reactions is therefore limited with shorter processing times. Nevertheless, optical emission spectroscopic measurements revealed that this processing leads to the lasting disappearance of nitrogen from the gas fill.

\subsection{Electrical circuitry}

The circuit used in our experiments is schematized in figure 2. The heart of the system lies in the twostage power supply whose driver is a function generator (top left, Wavetek model 39A) which supplies a low-voltage signal waveform of desired shape. This signal is then stepped-up to high-voltage levels (100 to $800 \mathrm{~V}_{\text {peak }}$ ) by a power amplifier (Spitzenberger EV600/C with UT600/C transformer), and applied to the lamp active electrode via a load resistor $R_{L}$. The purpose of the latter is to limit the lamp current after ignition has occurred. This resistor should therefore have a large value to prevent the lamp from reaching full regime.

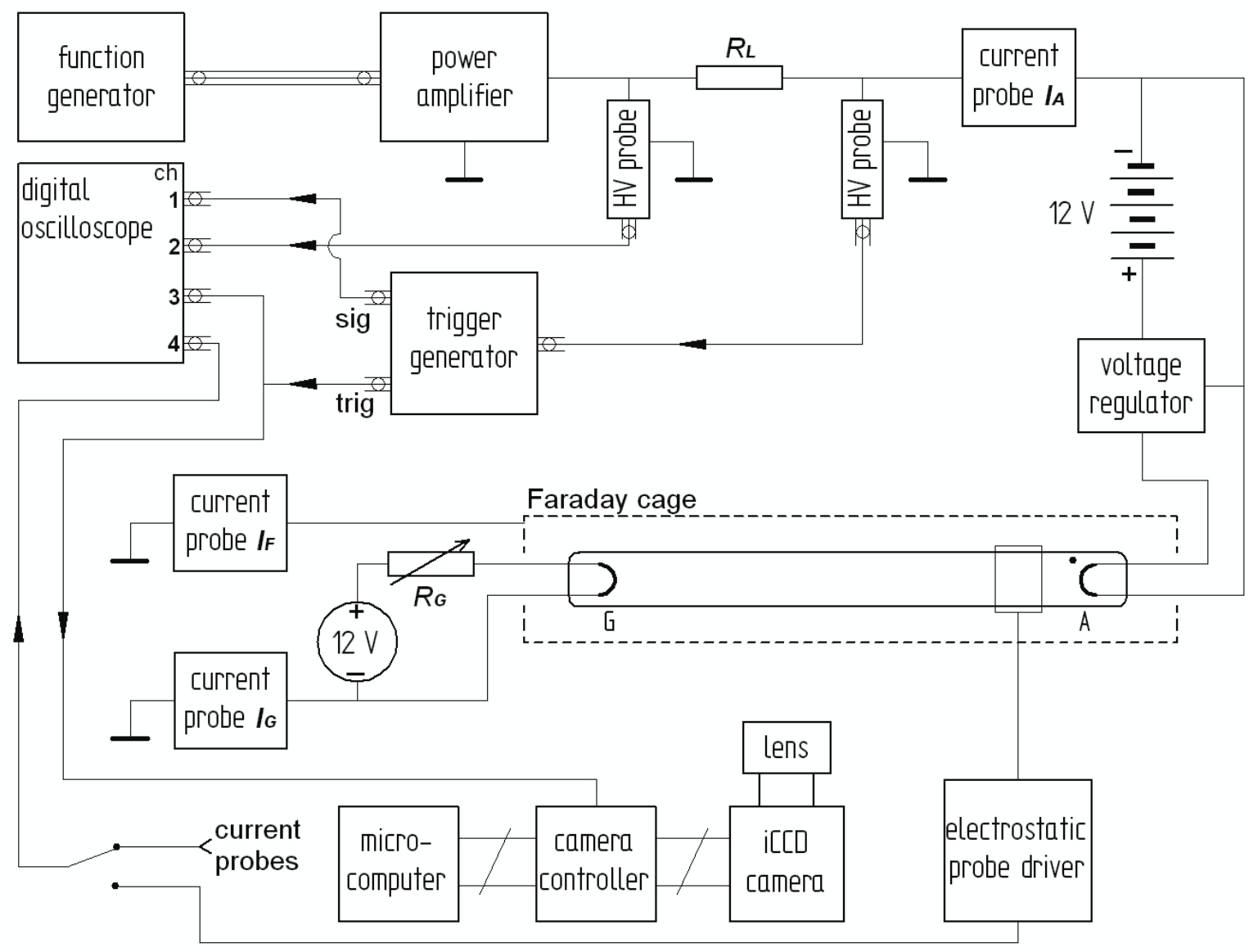

Figure 2: Configuration of the overall experimental set-up where the voltage waveform applied to the lamp active electrode $(A)$ is generated by a power amplifier driven by a function generator. The digital oscilloscope records potential waveforms from high voltage probes, from the electrostatic probe and from current meters. 
However, $R_{L}$ must also be kept at a low enough value because of the parasitic capacitance of the circuit which sets an RC time constant, itself preventing the application of sharp-edged voltage waveforms to the lamp. A value of $1 \mathrm{k} \Omega$ for $R_{L}$ was found to provide the best compromise between signal rise-time $(2 \mu \mathrm{s})$ and discharge current after ignition. Furthermore, the short duration of the applied voltage pulses ( $200 \mu$ s or less) prevents the full formation of an arc discharge in the lamp.

Both lamp electrodes can be heated to thermionic electron emission temperature via the circulation of currents in their tungsten filament. Different supply schemes are used depending on the electrode and its connection in the circuit. Work on the standardization of the pre-heating of fluorescent lamp electrodes was done [27] and uses the ratio $R_{h} / R_{c}$ between the hot and the cold resistance of electrodes to determine their average temperature according to the following relation [28]:

$$
T_{h}=T_{c} \cdot\left(\frac{R_{h}}{R_{c}}\right)^{0.814}
$$

It has been established that the normal operating point of electrodes should lie at an $R_{h} / R_{c}$ value of between 4 and 5.5, corresponding to an average electrode temperature of between $900 \mathrm{~K}$ and $1200 \mathrm{~K}$. The standard operating point for commercial lamps was chosen to be 4.75 , or a little more than $1000 \mathrm{~K}$, average temperature at which the active electrode was set (the grounded one is kept at $300 \mathrm{~K}$ ).

The lamp under investigation is located inside a Faraday cage designed to provide a protection against external electrostatic and atmospheric influences. This structure thus guarantees the quality, reliability and repeatability of the experiments. The lamp-cage structure is surrounded by three different kinds of diagnostic tools: a fast intensified CCD camera (Princeton Instruments $P I \bullet M A X: 512 R B$ ), a custom-designed electrostatic probe, and three custom-designed fast current probes. Only the first two diagnostics are treated in this article, with an emphasis on the capacitive probe. A trigger generator was specially designed to provide a low-jitter (8 ns) TTL reference signal locked on to the voltage applied to the lamp active electrode. This signal was employed to synchronize a digital oscilloscope (Tektronix TDS 754C) and the iCCD camera driver with the start of the lamp ignition. While the oscilloscope measures all potential waveforms in the circuit (including that retrieved from the electrostatic probe), the iCCD camera records a 2D map (512 pixels wide by 40 pixels high) of the optical emission associated with breakdown phenomena occurring in the lamp. The optical recording is integrated from 350 to $850 \mathrm{~nm}$ and no filtered measurement was done.

\subsection{Data recording and processing procedures}

The camera is linked to a microcomputer through a ST-133 controller (see figure 2) that serves the purpose of a delay generator driving the high voltage power supply of its image intensifier tube. This delay determines the time lag $\tau_{d}$ between the application of the lamp voltage (defined by the TTL output from the trigger generator) and the moment the lamp picture is shot with the camera. This controller also sets the shutter time $\tau_{s}$, whose value is defined through the computer interface. Although this time can be as brief as $2.5 \mathrm{~ns}$ the shortest time employed was $100 \mathrm{~ns}$ since this is the shortest resolution needed to follow the fastest ionization phenomena observed in our experiments. The evolution of breakdown in the lamp is recorded in the form of movie made via several shots taken with incremented values of the time delay $\tau_{d}$ ( $\tau_{s}$ being constant). Each of these single shot needs one complete electrical breakdown, and thus requires an excellent repeatability from ignition to ignition. The signal to noise level of the data is improved by image accumulation (20 to 50) at a given delay 
time. A space-time diagram of the light emission is then done by transforming the $2 \mathrm{D}$ optical data into 1D by summing along the vertical axis (normal to the lamp axis), and then by concatenating the 1D data along the time axis. The optical data are normalized on the shutter time and the number of accumulated pictured for the comparison of the optical intensity emission between different experiments (involving the same lamp).

The electrostatic probe measures the lamp wall potential on a localized $5 \mathrm{~mm}$-wide spot. 2D maps of the electrostatic potential are thus obtained by recording the probe signal as a function of time at different probe locations, thus giving a space-time diagram. In our experiments there are 30 measurement points equally spaced $5 \mathrm{~mm}$ apart along the lamp axis between its electrodes. This method also requires a lamp behavior which is identical from ignition to ignition and biased as little as possible by the location of the probe. The signal-to-noise ratio of the electrostatic data is also improved by averaging data over 32 samples at a given probe location. The relative variation of the measured signal from shot to shot is less than one percent.

The optical and electrostatic potential space-time diagrams are critical for a direct comparison and correlation between the data from the two diagnostic tools. The cross-comparisons are simplified via a data treatment procedure which detects the peak of the ionization wave profile, and then follows its evolution in time and space and retrieve the local value of a given parameter. The resulting time evolutions of these parameters are then used for direct and unambiguous comparisons.

\subsection{Lamp voltage waveforms}

The voltage applied to the lamp electrodes is not symmetrical. As shown in figure 2 one electrode is grounded (at left) while the active electrode (at right) is connected to the generator via the load impedance $R_{L}$. The high-voltage signal applied to the lamp active electrode consists of unipolar square pulses. The repetition rate of the signal is chosen to be $5 \mathrm{~Hz}$, leaving $200 \mathrm{~ms}$ between each signal burst, necessary to ensure a proper decay and diffusion of volume charges remaining from previous lamp ignitions. Experiments showed that a repetition rate of up to $10 \mathrm{~Hz}$ does not influence the development of the breakdown processes in the lamp. This is evidence that the remaining charges present just before the application of the voltage do not affect the lamp ignition [29].

Unipolar square pulses of positive or negative polarity provide the simplest case of study possible, allowing the investigation of fundamental ignition processes. The amplitude of the pulses ranges from 100 to $800 \mathrm{~V}$, with a rise-time set at $2 \mu \mathrm{s}$. The duration of the pulses is between 10 and $200 \mu$ s, chosen depending on the applied voltage and the formative lag time of the breakdown processes. In the general case the higher the applied voltage, the shorter the pulse duration.

On the one hand, pulses of negative polarity offer the most reliable way of starting breakdown reactions in the lamp. In the cathode phase the incandescent active electrode supplies plenty of electrons which contribute to the first electron avalanche in the gas between the electrode and the dielectric wall. A positive pulse, on the other hand, leads to starting mechanisms that rely on stochastic ionization by cosmic radiation or radioactivity (figure 3), resulting in a large spread of the statistical time of electron appearance [29] which will initiate an electron avalanche. This situation does not lead to accurate and repeatable observations in the sub-100 ns time scale. As a consequence all unipolar square pulses of positive polarity are preceded by a $2 \mu$ s negative pulse of 120 to $140 \mathrm{~V}$ amplitude (see figure 4) that serves the purpose of pre-ionizing the active electrode surroundings with thermallyemitted electrons from this temporary cathode. This pre-ionization pulse is kept at the lowest level possible enabling some ionization while the associated priming current level is too low to influence the overall breakdown conditions [30]. Yet, this measure ensures the repeatability of positive-polarity ignitions down to the $10 \mathrm{~ns}$ time scale. 

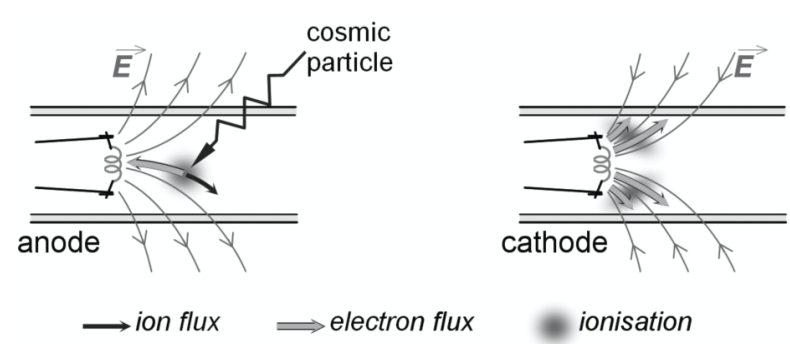

Figure 3: Influence of voltage polarity on the origin of the first ionization processes.

It is observed that the lamp does not fully ignite when a (too) weak pulse is applied to the active electrode. This situation leads to a memory effect which influences the breakdown processes, leading in the worst case to the lamp not properly igniting. This detrimental phenomenon is no longer observed when high-enough voltage levels are applied to ensure the development of a homogeneous plasma inside the lamp. Then, a way of solving the reproducibility problem associated with the study of failed ignitions consists in applying a second (resetting) pulse strong enough to completely ignite the lamp, after each experiment. The resetting pulse requirement is a negative potential square wave whose amplitude ranges from -850 to $-1200 \mathrm{~V}$ with a $10 \mu$ s duration, and applied $20 \mu$ s after the main pulse. Although the resetting procedure does not remove all the charges from the lamp wall, it allows the reduction of its level so a reliable and repeatable starting of the ionization waves can be achieved with the 200 ms extinction period between each ignition.
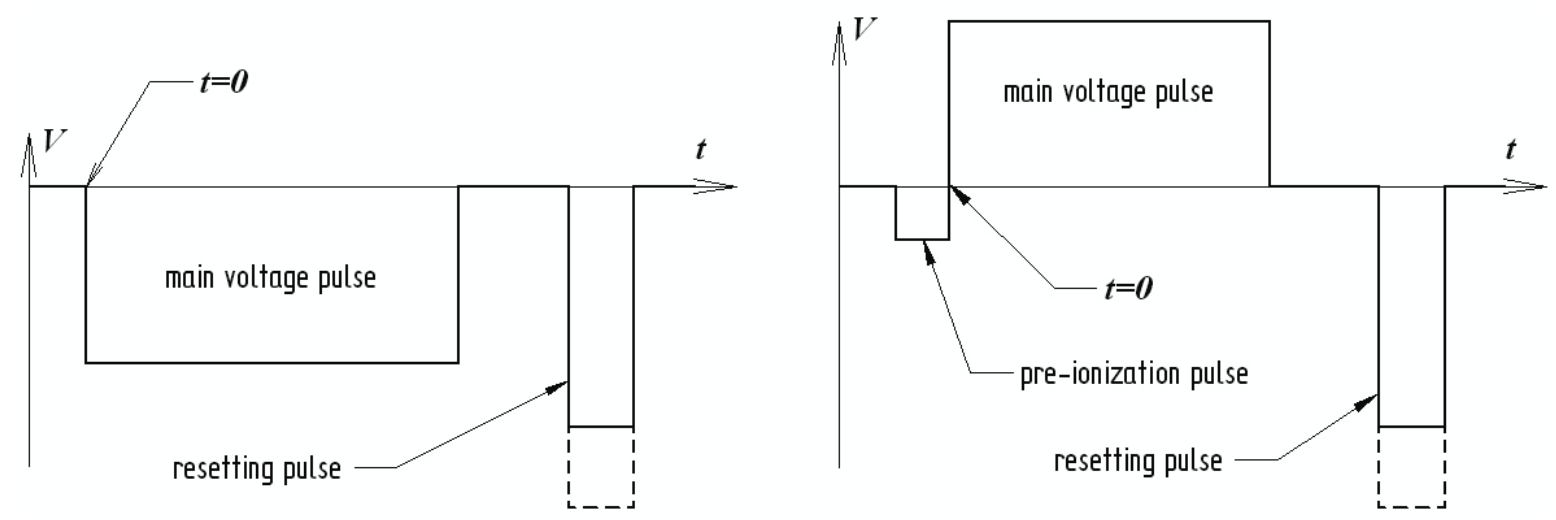

Figure 4: Schematic representation of the negative (left) and positive (right) unipolar waveforms applied to the lamp's active electrode. The resetting pulse has a variable amplitude which ranges from -850 to -1200 V depending on the main pulse level.

The voltage wave-forms displayed in figures 4 show that the resetting pulse is applied after the main pulse. This order prevents breakdown phenomena from being influenced by the decaying free charges created during the resetting phase. The $200 \mathrm{~ms}$ delay time between each main pulse is long enough for these parasitic volume charges to decay below a minimum level and do not affect the measurements.

\subsection{Shielding of the lamp}

As opposed to steady-state electrical discharges, phenomena occurring in discharge tubes during the ignition phase are of a transient nature. As a consequence these phenomena are influenced to a greater extent by the surrounding electrostatic environment and the presence of atmospheric moisture $[7,31$, 
32,33] and of light [34,35]. These influences are kept under control with the use of a cylindrical Faraday cage made of an electrically-grounded $60 \mathrm{~mm}$-diameter (outer) aluminum tube, primarily designed to precisely set the boundary conditions of the electrostatic potential surrounding the lamp (figure 5).



Figure 5: Cut-away view of the Faraday cage enclosing the linear lamp. A trolley located on top of the grounded cage supports the electrostatic probe sensor that can be displaced along the lamp. A semi-cylindrical glass window coated with indium-tin oxide (ITO - a semi-conductor) allows optical imaging of the lamp. The volume of the Faraday cage is continuously flushed with dry nitrogen to ensure a low level of humidity.

The lamp is located inside the Faraday cage within half a millimeter of the cage axis by two $15 \mathrm{~mm}$ thick PVC discs. After longitudinal centering, the lamp is secured with two Rilsan screws that also hold the PVC holders into place. No metallic parts are used to avoid any strong potential distortion near the lamp. The lamp linear capacitance profile is measured with a specially-designed instrument which yields a value of $37.8 \pm 0.6 \mathrm{fF} / \mathrm{mm}$, constant throughout the lamp between the electrodes (thus indicating a negligible impact of the PVC holders) and consistent with modeling.

The front of the Faraday cage has a $15 \times 160 \mathrm{~mm}$ aperture, covered by a soda lime silicate glass window spray-coated with a $310 \mathrm{~nm}$-thick layer of semi-conducting tin-doped indium oxide $\left(\mathrm{Sn}: \operatorname{In}_{2} \mathrm{O}_{3}\right.$ or ITO). This window is a semi-cylindrical section cut from the outer jacket of a low-pressure sodium lamp (Philips SOX $90 \mathrm{~W}$ ), where the ITO layer is used as an infrared mirror for heat conservation [35]. In our case the high charge carrier concentration and mobility in this ITO material is used for 
electrostatic shielding while enabling the optical imaging of the discharge lamp. A calculation involving the data from this material [36] applied to Drude's equation [37] yields an electron plasma cut-off frequency of $155 \mathrm{THz}$, corresponding to a dielectric-to-conductor transition at a wavelength of $1.9 \mu \mathrm{m}$ (shortwave infrared). The difference of refractive index between the semiconductor and the glass substrate leads to the occurrence of light interferences in the thickness of the ITO layer. This turns out to cause a variation of the light transmittance $\tau$ of the window as a function of the wavelength. The effect of this phenomenon is shown in the measured transmission spectrum in figure 6 .

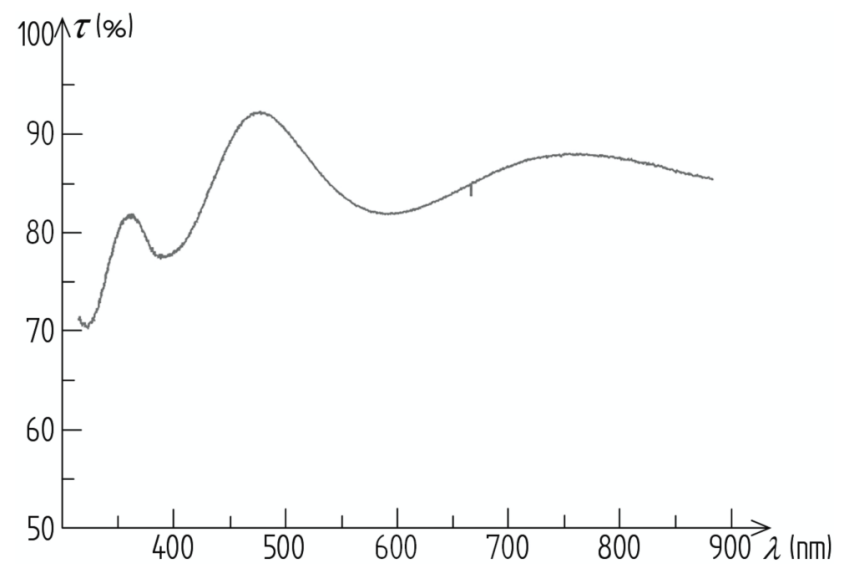

Figure 6: Absolute optical transmission spectrum of the ITO-on-glass window.

These interferences lead to a reflection coefficient that can exceed 20 percent in the blue region of the spectrum. The lamp was therefore placed co-axially to the Faraday cage tube and the window in order to reduce the effect of parasitic internal reflections. Finally, the inside surface of the Faraday cage tube was coated with a black mat paint to absorb any stray light. The purpose of this absorbing coat is also to keep the amount of parasitic light as low as possible so as to increase the signal-to-noise ratio of the iCCD data.

The Faraday cage is designed with a rectangular compartment, located on top, which houses a movable trolley holding the sensing end of the electrostatic probe. A 3 x $170 \mathrm{~mm}$ slit machined on top of the Faraday cage provides the necessary opening for the probe sensor to extend from the trolley to the lamp. The probe coupling electrode is precisely located along the lamp axis with an accuracy of half a millimeter. The electrical connection of the probe sensor to its driver is accomplished via a 20 $\mathrm{cm}$-long $50 \Omega$ coaxial cable, partially folded above the probe trolley inside the probe housing.

Thayer et al. [31] and later McFarland et al. [32] observed that the decrease of the lamp surface resistance due to water condensation could lead to an increase of the ignition voltage by a factor of 2.5 for certain mercury-containing fluorescent tubes. A similar influence of hygrometry was also observed in our experiments with argon lamps. The control of the water vapor level in the atmosphere is therefore important for the repeatability of the experiments. To this end the Faraday cage is continuously flushed with dry nitrogen so as to keep the moisture level as low as possible. This critical step ensures that the measured data are not dependent on the weather condition [7].

\subsection{Structure of the electrostatic probe}

The diagnostic tool needed for the electrostatic characterization of lamps in our experiments must meet the following requirements: a fast time response (75 ns), a moderately high spatial resolution (lower 
than $1 \mathrm{~cm}$ ), a low threshold level (1 $\mathrm{V}$ or best), a resolution of $1 \mathrm{~V}$ or better, a large dynamic range and a linear response from 1 to $1200 \mathrm{~V}$. Moreover this instrument should have a negligible impact on lamp ignition and a simple design that can be implemented inside the Faraday cage used in our experiments. Capacitive probes of the Aicher-Hammer type [38] are relatively simple to implement and meet most of the specifications. While this probe is a powerful diagnostic tool for the measurement of electrodeand plasma-related parameters of low-pressure discharge lamps during steady state operation [39], its structure invariably leads to very strong perturbations in the transient phase of ignition. In the general case two main causes are identified as the source of lamp perturbation observed with standard probe design:

- $\quad$ A large potential difference between the lamp and the probe's sensing ring leading to a large current drain from the ionization waves and to strong electric field distortions

- $\quad$ A zero potential gradient along the sensing ring support leading to a large electric field distortion in the lamp environment.

The design of Horstman et al. [16] consists in merging the capacitive probe with the cylindrical Faraday cage, and the connection of the probe ring to the ground via a $50 \Omega$ resistor keeps the probe potential close to that of the Faraday cage wall. Although such a scheme effectively solves the perturbation problem, it leads to two major issues: the spatial resolution of such instrument is directly dependant on the diameter of the Faraday cage, and the calculation of the lamp electrostatic potential requires an integrating procedure. The first issue requires the use of a Faraday cage tightly fitting the discharge lamp for sufficiently high spatial resolution to be realized, while the second issue requires a numerical method for its solution which is responsible for an increase of the noise level of the retrieved information.

All these problems are overcome with a new probe design having a sensing ring support made of a resistive material rather than a low-resistance conductor (e.g. plain metal). This modification allows two critical parameters to be tailored: the boundary potentials at the probe extremities and the shape of the potential gradient along the probe support. A proper design of the resistive support therefore permits a critical reduction in electric field distortion in the lamp-Faraday cage space. Furthermore, the voltage drop across the resistive support brings the sensing ring potential closer to that of the lamp, hence reducing the amount of current tapped from the breakdown waves despite an unchanged coupling capacitance between the probe and the lamp.

This resistive-capacitive probe (referred to as a $R C$ probe in the text) is connected to a current-tovoltage amplifier (figure 7) that sets the upper extremity of the resistive stand of the RC probe to a virtual ground potential (thus meeting one boundary condition at the Faraday cage wall). Moreover, this scheme limits the capacitive influence of the left coaxial cable (i.e. that connecting the probe to the amplifier) on the time response of the instrument. The main role of the amplifier is to process the probe current and provide an output voltage which is a real (i.e. non complex) fraction $F$ of the lamp potential. This way an integration procedure of data is no longer needed. In figure $7 \tilde{Z}_{H}$ and $\tilde{Z}_{F}$ are the complex impedances of the probe resistive support and of the negative feedback loop of the amplifier circuit, respectively. The capacitive coupling of the probe metal ring to the lamp is modeled as a capacitor $C_{P}$ whose value is measured to be $5.13 \pm 0.08 \mathrm{pF}$. Neglecting the parasitic capacitance between the coupling ring and the Faraday cage (estimated at $0.2 \mathrm{pF}$ ), the total complex transfer function $\tilde{H}$ of the probe-driver circuit is then expressed in equation (2) as: 


$$
\tilde{H}=\frac{\tilde{V}_{O}}{\tilde{V}_{L}}=-\frac{\tilde{Z}_{F}}{\tilde{Z}_{H}+\left(1 / j C_{P} \omega\right)}
$$

$\tilde{V}_{O}$ and $\tilde{V}_{L}$ in (2) are the complex system output voltage and the complex lamp potential (in respect to ground potential), respectively.

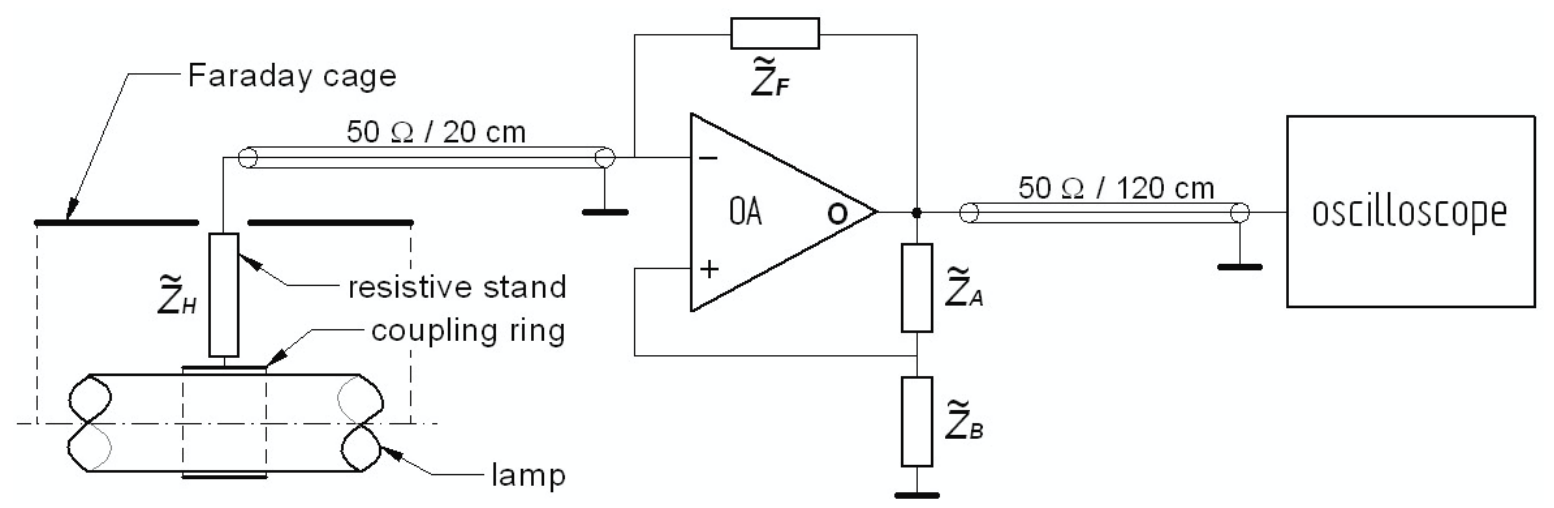

Figure 7: Electrostatic probe circuit design principle. The component OA is a fast differential amplifier whose negative feedback impedance converts the probe current into a voltage signal proportional to the electrostatic potential at the inner side of the lamp wall.

In equation (2) $\tilde{Z}_{F}$ is designed such that the circuit transfer function is equal to $-F$, set at $10^{-3}$ in our system. The purpose of the positive feedback impedances $\tilde{Z}_{A}$ and $\tilde{Z}_{B}$ is to ensure that the inverting input of the differential amplifier remains as close as possible to zero. These impedances must fulfill the following requirement for the system to properly work:

$$
\frac{\tilde{Z}_{B}}{\tilde{Z}_{A}+\tilde{Z}_{B}}=\frac{1}{A_{O}} \cdot\left(1+j \frac{\omega}{\omega_{1}}\right)
$$

where $A_{O}$ and $\omega_{1}$ are the open-loop gain and the first cut-off frequency of the operational amplifier, respectively. In the ideal situation the support that holds the sensing ring into position should have a potential profile along its length matching that of the free floating potential existing in the space between the lamp and the Faraday cage wall. In other words the resistance profile of the support must lead to an electrostatic potential that is the same as that of the free floating potential. Therefore, any current induced in the probe subsequent to any change of lamp potential will provide a potential gradient along the support structure that closely matches that of free space, thereby rendering the probe transparent to the lamp.

For mechanical reasons the probe is made of four different $5 \mathrm{~mm}$-long carbon film resistors buttsoldered at their rims (figure 8). This assembly forms a $20 \mathrm{~mm}$-long cylindrical structure with one end soldered to the ring sensor ( $5 \mathrm{~mm}$ wide, $12.5 \mathrm{~mm}$ inner diameter, $0.5 \mathrm{~mm}$ thick) and the other end spliced to a male bayonet base (not shown in the figures). This base is plugged into a female socket located in the probe rack trolley (see figure 5), connecting the probe to its electronic driver via a 20 $\mathrm{cm}$-long $50 \Omega$ coaxial cable. The value of each resistor composing the stand is calculated such that the global stepped potential profile of the probe support follows the potential profile of free space as 
closely as possible (see figure 8). To this end the analytical form of the radially-dependent floating potential is derived in an unperturbed space between the lamp inner wall surface and the Faraday cage.

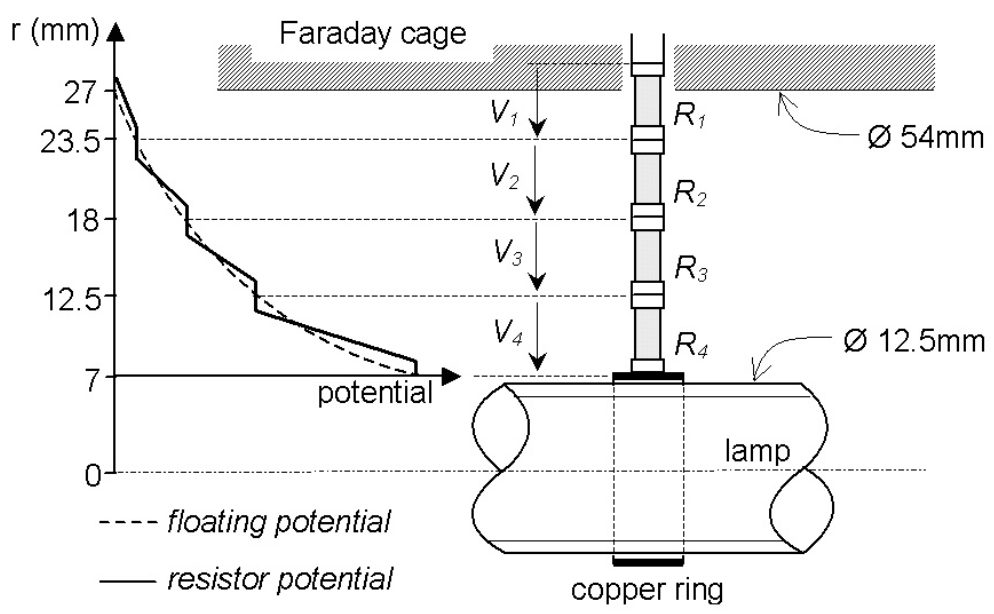

Figure 8: Configuration of the resistive probe support. The floating potential in the space between the lamp and the wall of the Faraday's cage is drawn (dashed lines) alongside with that of the resistor holder (solid lines).

In this calculation it is assumed that the lamp is homogeneously charged and forms a coaxial capacitor with the Faraday cage, which logically leads to a logarithmic form for the expression of the floating potential:

$$
\phi(r)=V_{L} \cdot \frac{-\varepsilon_{g} \ln \left(R_{f c} / r\right)}{\varepsilon_{n} \ln \left(R_{i} / R_{o}\right)+\varepsilon_{g} \ln \left(R_{f c} / R_{o}\right)}
$$

Expression (4) is valid only in the space between the Faraday cage and the lamp outer wall. In this formula $R_{f c}, R_{o}$ and $R_{i}$ are the radii of the Faraday cage, of the lamp outer wall and inner wall, respectively. $\varepsilon_{n}$ and $\varepsilon_{g}$ are the relative dielectric permittivity of the nitrogen gas fill (1.0) and of the glass (6.0), respectively. The lamp potential $V_{L}$ in respect to ground is defined at the inner wall surface of the lamp at $r=R_{i}$. The values of each resistor relative to the total ohmic impedance $R_{H}$ of the support structure is calculated from (4) by setting $V_{L}$ as an arbitrary constant, although it is a time- and spacedependant variable. This procedure is permitted since the relative values of the resistances do not depend on the nature and value of $V_{L}$. The calculations lead to $R_{I}=10.0, R_{2}=19.3, R_{3}=26.2$ and $R_{4}=44.5$ $\%$ of $R_{H}$. The total resistance $R_{H}$ is chosen so the resistive-capacitive network formed by the probe and the different capacitances (lamp-coupling and parasitic) leads to several specific requirements:

- $\quad$ the probe sensor ring potential must be close to $92.9 \%$ of $V_{L}$

- $\quad$ the time constant of the resistive-capacitive network should exceed $100 \mu \mathrm{s}$

- $\quad$ the probe current must be larger than the input bias current of the amplifier

The first requirement is directly drawn from expression (4) and the knowledge of the radial position of the sensor ring. The second requirement is arbitrarily set so any significant changes of the probe potential will happen long after the breakdown processes have occurred in the lamp. It should be noted that this time constant does not affect the output signal of the probe driver since the negative feedback 
impedance of the differential amplifier compensates for the time behavior of the sensing part of the RC probe. Finally, the third requirement is needed for the proper operation of the differential amplifier. This last point does not impact on the capacitive current drawn from the nascent discharge as long as the first requirement is met, in which case this capacitive current will be close to that in free space. Calculations lead to a value for the total support resistance of $R_{H}=23.52 \mathrm{M} \Omega$. The resulting time constant is $128.3 \mu$ s and the probe current for $\Delta V_{L}=1 \mathrm{~V}$ is $41.7 \mathrm{nA}$, effectively exceeding the $10 \mathrm{pA}$ bias current of the amplifier.

Ignition tests at $-400 \mathrm{~V}$ with the argon lamp reveal that the variation of the formative lag time of breakdown as a function of the RC probe position does not exceed 5\% of the lag time of the lamp without probe. This result is obtained despite the field gradient caused by the sharp edges of the sensing ring. Comparatively, a standard capacitive probe was found to cause a variation of the formative lag time of $40 \%$ in the same operating conditions. It can therefore be concluded that the RC probe design is effective and the sharp features of its sensor have a negligible impact on lamp ignition.

\subsection{RC probe data treatment and processing}

Data recorded from the RC probe system undergo five distinct processes whose order is presented in the algorithm of figure 9. Firstly, raw data are cropped in time in order to select the proper region of interest. Then, the cropped information is compressed in order to increase the time step by a predetermined factor so as to match the time step of optical data, thus enabling a direct comparison between diagnostics. Since the time step of the raw electrostatic data are in the order of $10 \mathrm{~ns}$, several times less than the time step of the optical data (100 ns of more), a simple averaging method is used for this data compression.

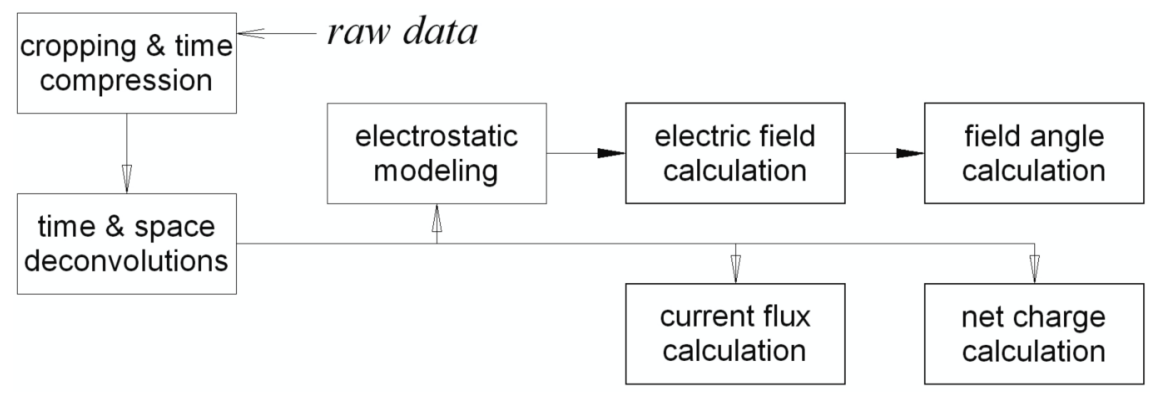

Figure 9: Global algorithm of the processing of raw data retrieved from electrostatic measurements done with the $R C$ probe system. Computation steps in bold rectangles provide data that are displayed and saved in output files.

In a third step the information is deconvolved because the recorded signal comprises in fact both the physical event and distortions introduced by the amplifier circuit and the sensing probe. Although the driving circuit has feedback impedances optimized to give an output as undistorted as possible, the internal delays and bandwidth limitations of the amplifier as well as parasitic impedances in the global circuit give the system a non-Dirac impulse response. As a result the temporal resolution of the whole system is increased to several microseconds, with some distortions in the output signal. In terms of spatial resolution the stray electric field at the edges of the sensing ring increases the resolution beyond the $5 \mathrm{~mm}$ of the physical width of this electrode. Specific deconvolution schemes based on the Jansson-van Cittert method [40,41] combined with Savitsky-Golay convolution filtering $[42,43]$ were 
developed to efficiently restore a $75 \mathrm{~ns}$ time resolution and a $5 \mathrm{~mm}$ space resolution from the recorded data.

Finally, parameters of interest such as the radial and axial components of the electric field at the lamp surface, the displacement currents flowing between the lamp and the Faraday cage and the net electrostatic charge are computed from the pre-processed data. If on the one hand the calculation of the axial component $E_{z}$ of the electric field involves only a differentiation along the space axis (i.e. axial direction of the lamp) of the electrostatic data, the derivation of the radial component $E_{r}$, on the other hand, is less trivial due to the lack of spatial information in the radial direction of the recorded data. Although expressions (4) provides the potential profile as a function of radius, this formulas cannot be employed for directly computing $E_{r}$ due to its validity in the ideal case of a lamp homogeneously charged along its whole length. Since the charging of the lamp is not homogeneous during breakdown, a full electrostatic model of the lamp-Faraday cage system was developed to compute the map of the floating potential surrounding the lamp. Then, a gradient operation was performed for the retrieval of both E-field components. A resulting useful information is the angle of the $\mathrm{E}$ field vector:

$$
\angle E=\tan ^{-1}\left(\frac{E_{z}}{E_{r}}\right)
$$

The field angle calculated via (5) is defined at the lamp outer wall (i.e. not at the lamp axis) and is equal to zero when oriented from the lamp to the Faraday cage, it is oriented from the active electrode to the grounded one at $+90^{\circ}$ and it is $+/-180^{\circ}$ when directed from the dielectric wall toward the lamp axis.

The calculation of the displacement current $\left.I_{D}\right|_{m}$ flowing between the lamp and the Faraday cage at location $m$ along the lamp axis, between the electrodes, provides some useful information about the charging dynamics of each section of lamp as a function of time. Since the capacitive coupling value between the Faraday cage and each $5 \mathrm{~mm}$ lamp section is known from measurements, the computation of the displacement current as a function of time and axial position is done as follow:

$$
\left.I_{D}\right|_{m}(t)=\left.\frac{d V_{L}(m, t)}{d t} \cdot C\right|_{m}
$$

Where $\left.C\right|_{m}$ is the capacitance value coupling the faraday cage to the lamp along the length $L_{P}$ covered by the capacitive probe (i.e. $5 \mathrm{~mm}$ ), and $V_{L}(m, t)$ is the lamp electrostatic potential as a function of time, with both parameters defined at location $m$. The parameter which is plotted in the diagrams in this work is the linear displacement current $I_{d l}$ which is related to $I_{D}$ by the following relation:

$$
\left.I_{d l}\right|_{m}(t)=\left.I_{D}\right|_{m} \cdot L_{P}^{-1}=\frac{d V_{L}(m, t)}{d t} \cdot C_{l}
$$

Where $C_{l}$ is the lamp linear capacitance equal to $37.8 \mathrm{fF} / \mathrm{mm}$ and constant along the lamp. Finally, the total displacement current $I_{D T}(t)$ flowing from the whole lamp (i.e. from the $150 \mathrm{~mm}$-long area covered by the RC probe) to the grounded Faraday cage is computed by summing each individual displacement current $\left.I_{D}\right|_{m}$ over the length of the lamp:

$$
I_{D T}(t)=\left.\sum_{m=1}^{N} I_{D}\right|_{m}(t)
$$


With $N$ the total number of measurement points, which in our system is 30 . At last, the final parameter of interest considered in this article is the amount of net electrostatic charge at each individual 5-mm lamp section that results from the potential charging of the lamp. These values are also retrieved from the measured capacitive coupling of the lamp to the Faraday cage. The time evolution of the net charging of each individual lamp section is computed as follows:

$$
\left.Q\right|_{m}(t)=\left.V_{L}(m, t) \cdot C\right|_{m}
$$

The total net charge of the lamp (i.e. over the $150 \mathrm{~mm}$-long volume covered by the RC probe) is calculated by the summing net charge from each individual lamp section:

$$
Q_{T}(t)=\left.\sum_{m=1}^{N} Q\right|_{m}(t)
$$

All the parameters developed in this section are functions of time and space and are therefore presented in space-time diagrams in a way similar to the diagrams of the optical emission and the lamp electrostatic potential. This method of data display permits the comparison side-by-side of different parameters. The data of the E field angle vector are displayed only above a certain threshold value for the E field amplitude.

\section{Ignition processes at negative potentials}

In the negative ignition experiments the amplitude of the voltage pulse applied to the active electrode ranges from $-200 \mathrm{~V}$ to $-800 \mathrm{~V}$ with measurements done with $100 \mathrm{~V}$ steps. The time resolution of the processed optical and electrostatic data is $500 \mathrm{~ns}$ for -200 and $-300 \mathrm{~V}$ and is $100 \mathrm{~ns}$ for higher voltage amplitudes.

\subsection{Overview of phenomena}

The space-time diagrams of the measured light emission, of the electrostatic potential and of the calculated linear displacement currents are presented in figure 10 in the case of a successful ignition at - 500V. Several optical phenomena are observed upon the application of the voltage on the active electrode. First, a luminous channel associated with the first ionisation wave forms at the active electrode and starts to move toward the opposite (ground) electrode at $1.5 \mu \mathrm{s}$. Once it has completed its propagation $(6 \mu \mathrm{s}$ at $-500 \mathrm{~V})$ a second luminous channel associated with a second ionisation wave (the return stroke) develops from the ground electrode back toward the active electrode, drawing a continuous plasma channel in its wake which finally bridges both electrodes $(8.5 \mu \mathrm{s}$ at $-500 \mathrm{~V})$. At this point the luminous intensity of this homogeneous channel increases in time until a stable discharge operation is reached, stabilized by the series impedance connecting the lamp to the power supply (figure 2). These optical observations are consistent with the literature [15,16,17,18].

During the voltage ramp-up phase $(0-2 \mu \mathrm{s})$ the first ionisation processes occur around the active electrode due to thermionically-emitted electrons that are pulled by the local E field oriented from the electrode toward the Faraday cage. Since the distance from the lamp to the grounded tube is much smaller than the inter-electrode distance, the electric field is then directed in the radial direction from the electrode as shown in the field angle map of figure 11 up to $1.5 \mu \mathrm{s}$. As the electrons progressively cover the inside surface of the wall, the potential gradient and the region of ionisation shift ahead of the active electrode and the pre-breakdown wave starts its propagation at $1.5 \mu \mathrm{s}$. This first ionisation process leads to the formation of a luminous channel which progressively develops from this electrode 
and propagates toward the ground electrode (figure 10). This wave is associated with a region of strong potential gradient responsible for the formation of a local front of high electric field strength (figure 11) which then drives the ionisation processes there. This local ionisation leads to the charging of the lamp which can be seen from the map of displacement current in figure 10. Since the start of its propagation the $\mathrm{E}$ field vector angle of the wave front shifts at $1.5 \mu \mathrm{s}$ from $-180^{\circ}$ to $-120^{\circ}$ and keeps this angle during the entire wave propagation. This angle indicates that the axial component of the $\mathrm{E}$ field is stronger than its radial component (an equal or stronger radial component would lead to a field angle of between $-135^{\circ}$ and $-180^{\circ}$ ). It was previously believed that the close spacing between the Faraday cage and the lamp would lead to a stronger radial component, but the potential gradient along the lamp axis in the wave front region leads to the contrary. Nevertheless, the E field becomes radial in nature just after the passage of the wave front as a result of a decreased axial potential gradient in the wake of the pre-breakdown wave.
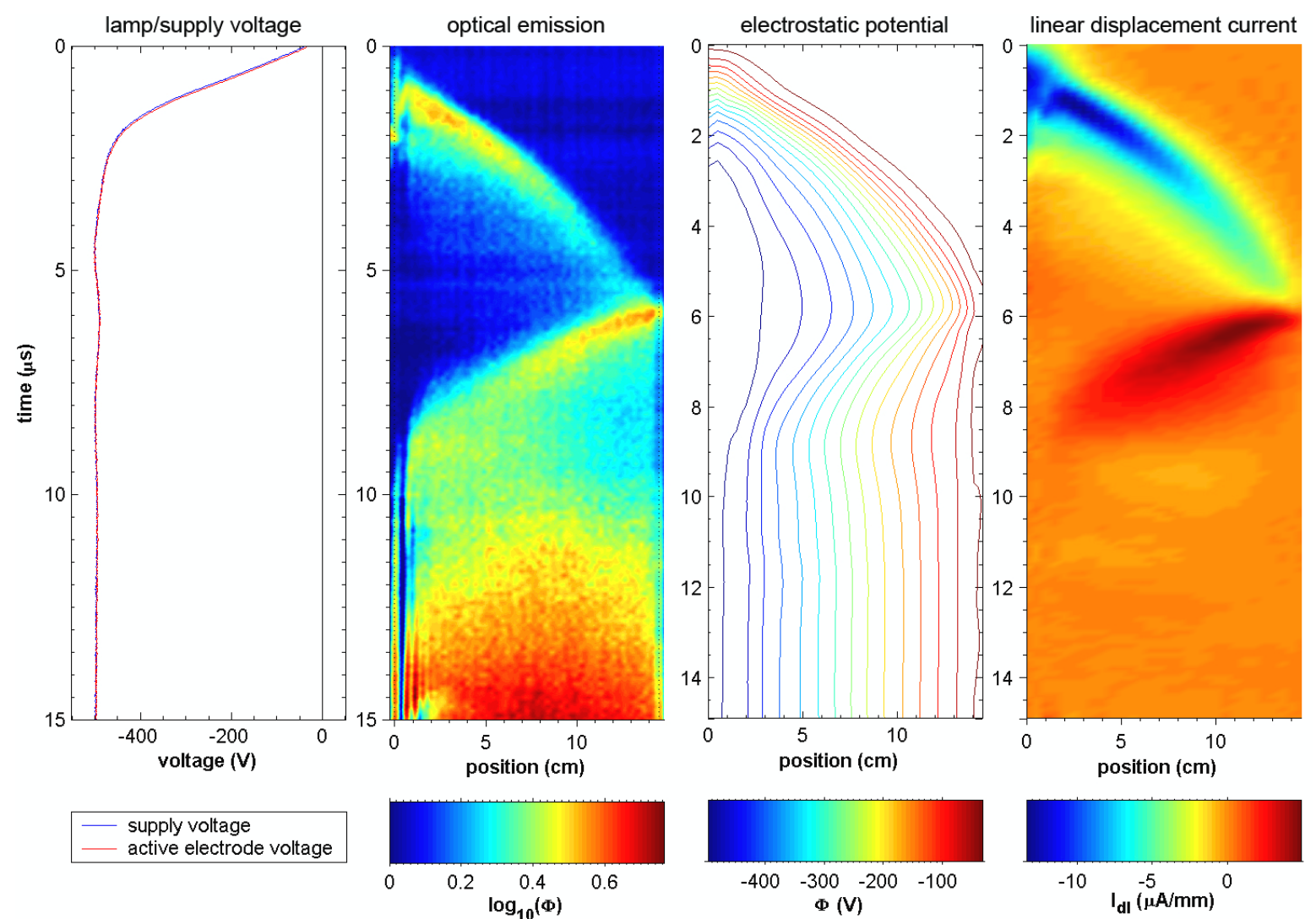

Figure 10: Space-time diagrams of the evolution of the measured light emission, of the net electrostatic potential and of the calculated linear displacement current (positive from the lamp to the Faraday cage) associated with breakdown phenomena occurring in 3 Torr argon at $-500 \mathrm{~V}$. The active and the ground electrodes are located at 0 and $14.5 \mathrm{~cm}$, respectively.

The E field amplitude and the displacement current present the same profile and follow the same evolution in space and time as the measured light emission from the ionisation wave, which is evidence of the correlation between these different parameters. It is observed that the luminous intensity of the pre-breakdown wave decreases both in time and space during its propagation, and this evolution is correlated by a decrease of the axial E field strength (figure 11). This leads to a gradual 
decrease of the ionisation rate in the wave front, thus resulting in lower lamp charging rates and in a decreasing displacement current, as seen in figure 10. The wake of the pre-breakdown wave presents a weaker potential gradient than in the wave front, resulting in a lower E field strength there and a lowers level of light emission in the ionised gas.
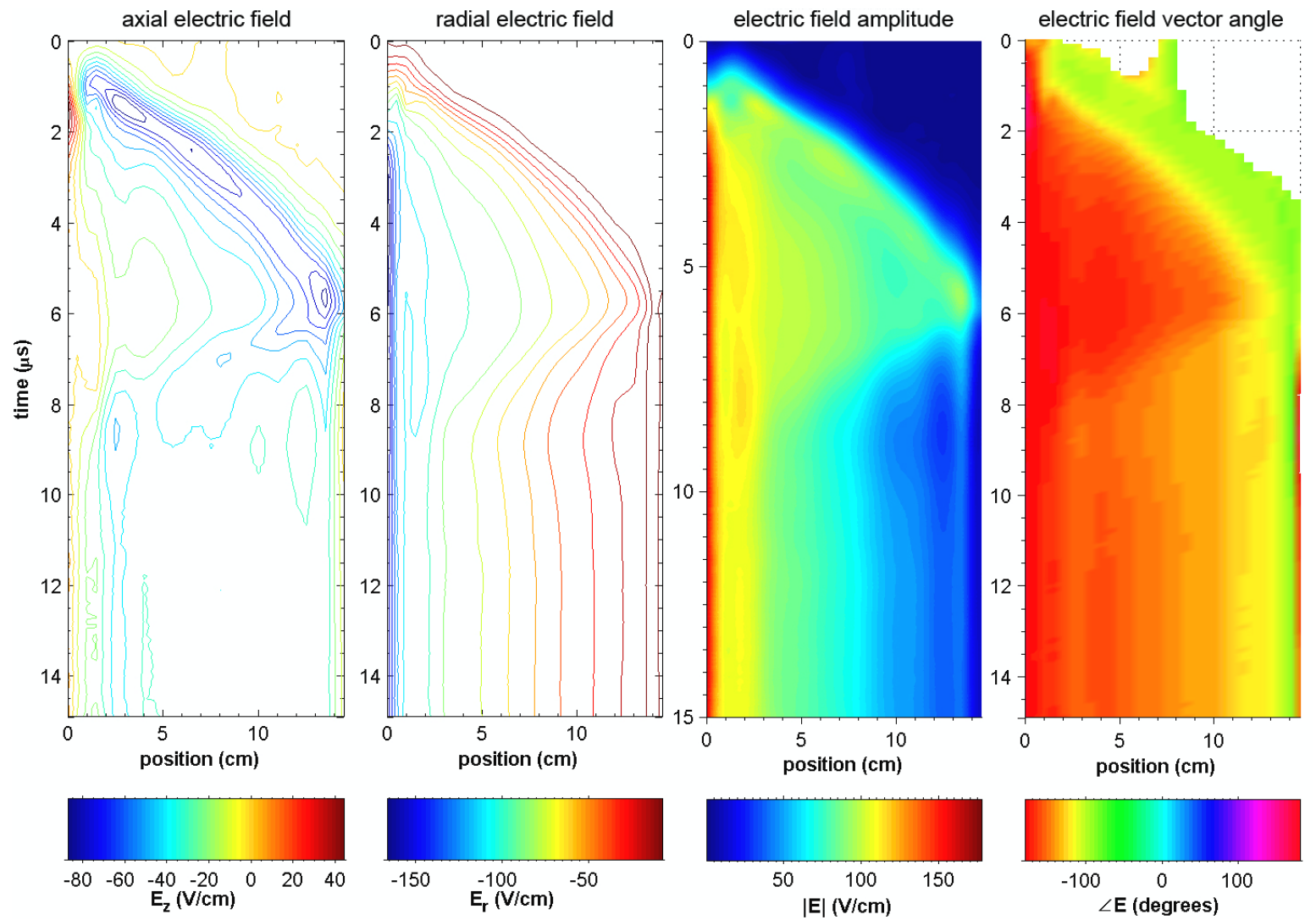

Figure 11: Space-time diagrams of the evolution of the axial electric field (positive from the active to the ground electrode), of the radial electric field (positive from the lamp to the Faraday cage), of the electric field amplitude and vector angle (null when oriented from the lamp wall to the Faraday cage, and oriented from the active to the ground electrode at $+90^{\circ}$ ) associated with breakdown phenomena occurring in 3 Torr argon at $500 \mathrm{~V}$. The active and the ground electrodes are located at 0 and $14.5 \mathrm{~cm}$, respectively.

When the pre-breakdown wave reaches the ground electrode the E field angle vector then becomes fully axial (angle of $-90^{\circ}$ at $6.5 \mu$ s and $13.5 \mathrm{~cm}$ in figure 11). This is due to a large potential difference and a decreasing separation length between the pre-breakdown wave and the ground electrode. The resulting local $\mathrm{E}$ field increase leads to the formation of the return stroke which propagates back toward the active electrode. The optical emission associated with this wave has similar features as those of the first wave, like a decreasing light intensity and a profile that becomes steeper in time and space. The return stroke is also associated with potential gradient change between the electrodes (figure 10) which results from the partial discharging of the lamp and leads to a reversed displacement current whose profile has the same shape and trend as that of the measured light emission. The local $\mathrm{E}$ field associated with the wave front is essentially radial in nature since its axial component has a much lower amplitude than in the pre-breakdown wave front. This component decreases further during the wave propagation and eventually leads to an inter-electrode region that has a relatively homogeneous 
and weak axial $\mathrm{E}$ field (figure 11). The slight supply voltage dip observed from 5 to $8 \mu$ s (figure 10) is not caused by the wave propagation since its associated current is too low to affect the circuit.

The formation of electrode sheaths is observed during the development of ignition processes, with the first one occurring in front of the active electrode, driven as a cathode. This process starts at $1 \mu \mathrm{s}$ with the reversal of the axial $\mathrm{E}$ field which peaks just before $2 \mu \mathrm{s}$ and wanes out at $3 \mu \mathrm{s}$ as a result of wall charge build up. This then leads to the formation of a stable and strong radial $\mathrm{E}$ field in the $0-5 \mathrm{~mm}$ region (figure 11) which remains present during the entire duration of the measurement.

The second electrode (anode) sheath develops at the end of the propagation of the pre-breakdown wave $(5.5 \mu \mathrm{s})$. The closing of the gap between the wave front and the ground electrode leads to the formation of a zone of high axial $\mathrm{E}$ field strength which then evolves into a $1 \mathrm{~cm}$-thick region of axial E field gradient which binds the nascent plasma to the anode. This region has a relatively weak $\mathrm{E}$ field strength of about $15 \mathrm{~V} / \mathrm{cm}$ whose vector angle remains at $-90^{\circ}$ (figure 11 in the $13.5-14 \mathrm{~cm}$ region) necessary to draw and collect electrons from the weakly ionised gas.

A negative glow forms at the location of the cathode as soon as the return stroke reaches the active electrode at $8.5 \mu \mathrm{s}$. The appearance of this feature is evidence of an electron current which starts to flow from the cathode as soon as the return stroke has bridged the lamp's electrodes. From this point a Faraday dark space also forms over a length of $2 \mathrm{~mm}$ between the negative glow and the ionised gas column. Some plasma striations are also observed in the vicinity of the cathode region (optical map, figure 10) whose origin is most likely the Frank and Hertz phenomenon [44].

\subsection{Wave propagation characteristics}

The propagations of the pre-breakdown and of the return stroke are investigated from optical measurements and from the calculated axial E field strength obtained from the measured electrostatic data. The optical (CCD) and electrostatic (RCP) position of the waves are thus defined at the peak of light intensity and of E field strength, respectively. The results are presented in figure 12 and show that both diagnostics yield similar trends in the propagations for each voltage level applied to the active electrode.

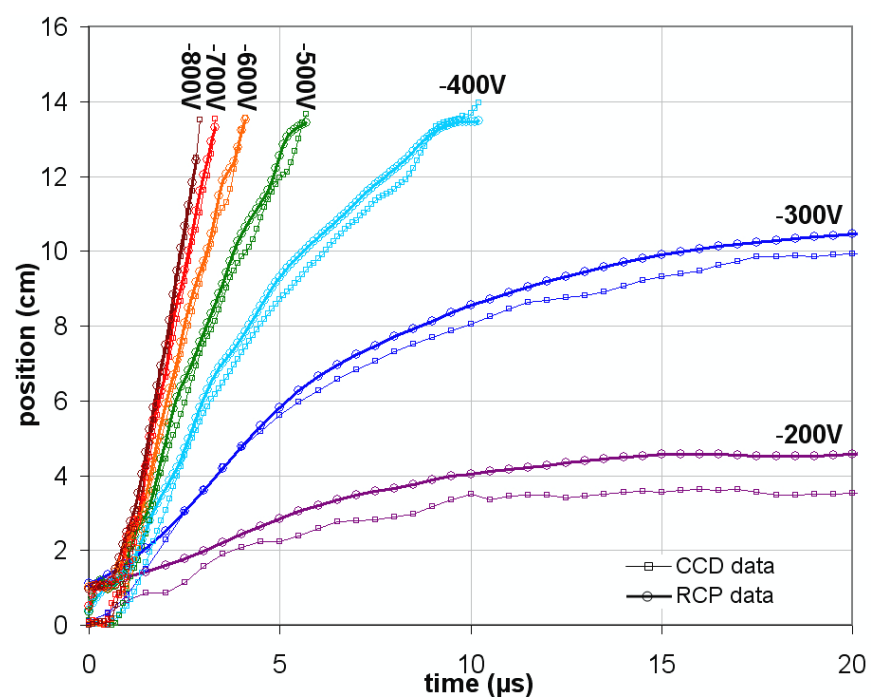

Figure 12: Measured position of the optical and of the E field peak of the pre-breakdown wave front in 3 Torr argon as a function of time and of the applied voltage amplitude. Ignitions below $-400 \mathrm{~V}$ are unsuccessful. The active and the ground electrodes are located at 0 and $14.5 \mathrm{~cm}$, respectively. 
There is however a difference in absolute values between CCD and RCP data, leading to the observation that the peak of $\mathrm{E}$ field strength in the pre breakdown wave is located ahead of the peak of light emission. This offset depends on the applied voltage and reaches its largest value at $-200 \mathrm{~V}$ with a shift of $0.9 \mathrm{~cm}$. Considering a proportional relation between the reaction rate coefficient $K_{e x}$ of argon excitation by electron impact and the observed light emission $I_{\Phi}$ in the pre-breakdown wave front, we can then derive the following relation:

$$
I_{\Phi} \propto n_{e} n_{A r} K_{e x}
$$

Where $n_{e}$ and $n_{A r}$ are the densities of electrons and of argon atoms, respectively. The excitation rate is expressed as a function of the electron energy $\varepsilon_{e}$ and mass $m_{e}$, its energy distribution function $f\left(\varepsilon_{e}\right)$ and the cross section of argon atom excitation by electron impact $\sigma_{e x}$ such that:

$$
K_{e x}=\int_{0}^{\infty} \sigma_{e x} \sqrt{\frac{2 \varepsilon_{e}}{m_{e}}} \cdot f\left(\varepsilon_{e}\right) \cdot d \varepsilon_{e}
$$

In the region of peak electric field strength the reaction rate coefficient $K_{e x}$ is bound to be higher than in the wake of the ionisation wave where the axial $\mathrm{E}$ field strength is lower and leads to a lower mean electron energy. However, the density of free electrons in the region of peak field is not high enough to maximize $I_{\Phi}$, this optimum occurs behind this region where there is an intermediary level of electron density and of reaction rate. The decrease of the offset between the peak of E field and of light emission is an indication that the evolution of electron density in the wave front becomes steeper for higher applied voltage levels.

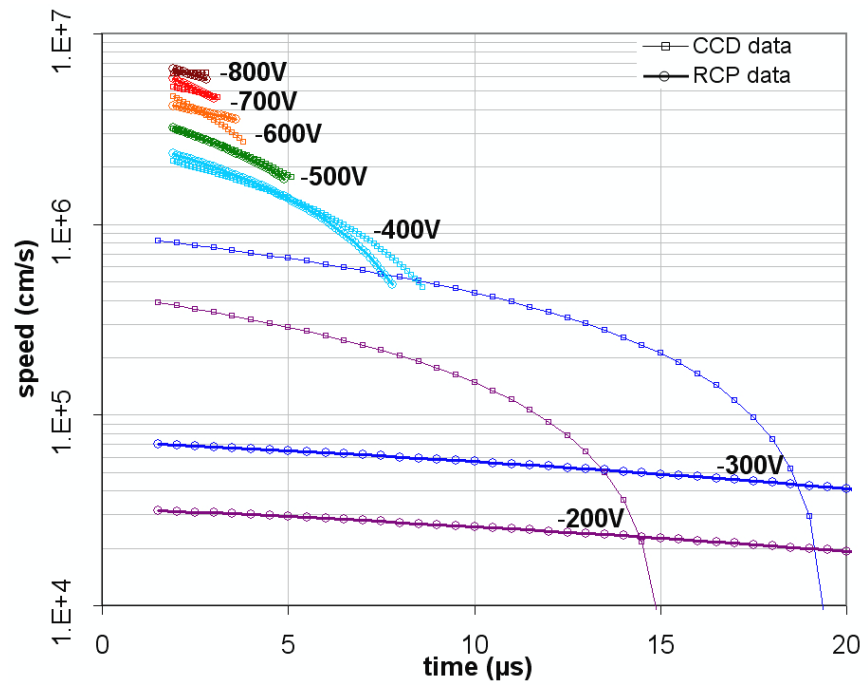

Figure 13: Calculated velocity of the optical and of the E field peaks of the prebreakdown wave front in 3 Torr argon as a function of time and of the applied voltage amplitude. Ignitions below $-400 \mathrm{~V}$ are unsuccessful.

Figure 12 shows that the time it takes for the pre-breakdown wave to reach the ground electrode at $14.5 \mathrm{~cm}$ increases for lower applied voltage. Ignitions below $-400 \mathrm{~V}$ lead to a wave which reaches an asymptote below $12 \mathrm{~cm}$ and thus fails to reach the ground electrode, leading to an ignition failure of 
the lamp. This observation leads to the first two rules for a successful lamp ignition: the applied voltage amplitude must be high enough and the duration of the unipolar voltage pulse must be long enough so as to enable the pre-breakdown wave to complete its propagation and reach the grounded electrode. The propagation of this wave is in all cases non linear and has a slope which gradually decreases in time.

The velocity of the pre-breakdown wave is calculated from the derivation of a second order polynomial function fitted to the wave position data. The calculated velocities are plotted in figure 13 and show that the initial wave velocity for successful ignitions ranges from $23 \mathrm{~km} / \mathrm{s}$ at $-400 \mathrm{~V}$ to $65 \mathrm{~km} / \mathrm{s}$ at $-800 \mathrm{~V}$ and decreases in time with a magnitude which increases for lower applied voltage $V_{A}$ values.

It is found that the peak and the average wave speed are linearly proportional to $V_{A}$. The results derived from optical and from electrostatic data are again coherent between each other, showing similar trends except for the failed ignitions at -200 and $-300 \mathrm{~V}$. At these low voltages there is a difference as high as one order of magnitude in the initial velocity, with that of the peak of $E$ field being higher and decreasing faster over time than the velocity of the optical peak. This behaviour is due to the extinction of the waves and the displacement of the E field peak away from that of light emission due to space charge build-up (section 3.5).

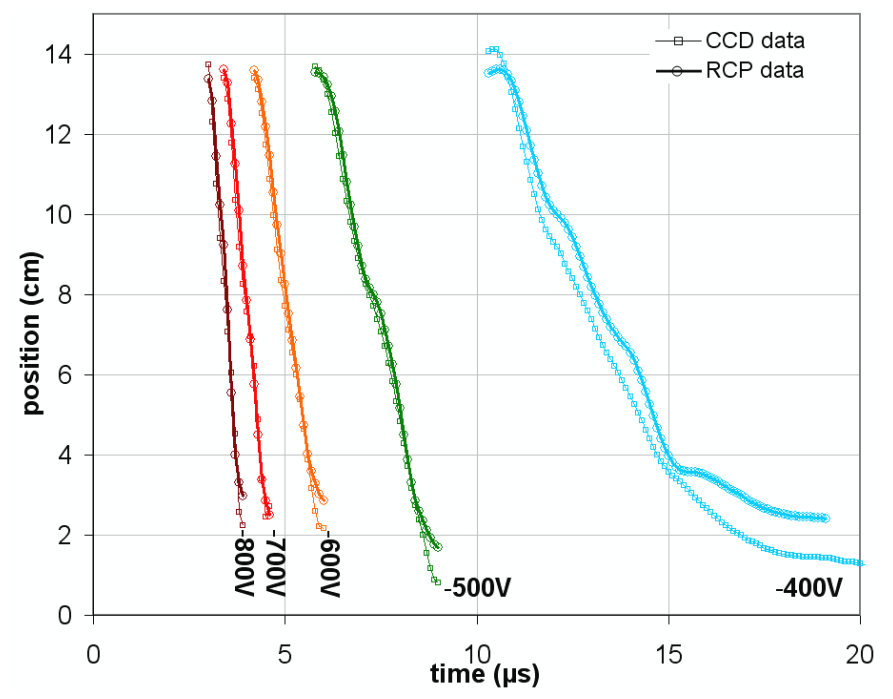

Figure 14: Measured position of the optical and of E field peaks of the return stroke wave front in 3 Torr argon as a function of time and of the applied voltage amplitude. The active and the ground electrodes are located at 0 and $14.5 \mathrm{~cm}$, respectively.

The measured positions of the return stroke (figure 14) show a more linear propagation over time than for the pre-breakdown wave and feature a steeper slope. An offset between the peak of E field strength and of light emission is also observed, although the shift is smaller in the return stroke for $V_{A}$ higher than $-400 \mathrm{~V}$. This is an indication that the evolution of the electron density in this wave front is less steep than in pre-breakdown wave fronts, which is expected since the return stroke evolves in a preionized medium while the first wave has to propagate in a neutral gas. The velocities derived from optical and electrostatic data (figure 15) agree relatively well between each others and shows peak values that are higher than for the pre-breakdown wave by a factor $1.7(-400 \mathrm{~V})$ to $2.6(-800 \mathrm{~V})$. The evolution of the return stroke velocity depends on the applied voltage level. At $-800 \mathrm{~V}$ the velocity curve of figure 15 presents only one peak, while there are two peaks from -500 and $-700 \mathrm{~V}$ with a 
minimum in the middle of the wave propagations, and the number of peaks finally rises to three at $400 \mathrm{~V}$. The origin of the velocity modulation in time and space could be spatial fluctuations in the charge density of their propagation medium.

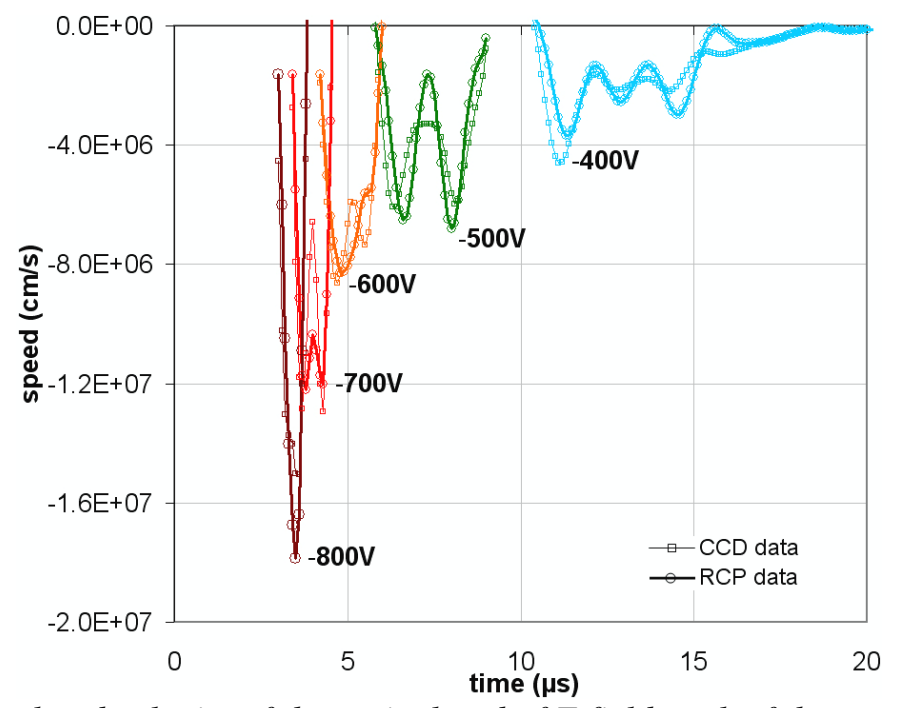

Figure 15: Calculated velocity of the optical and of E field peak of the return stroke wave front in 3 Torr argon as a function of time and of the applied voltage amplitude.

\subsection{Electric field and potential evolutions}

The electric field strength (figure 16) and potential (figure 17) of the pre-breakdown wave front steadily increase during the $2 \mu$ s rise time of the active electrode voltage, and reach an $\mathrm{E}$ field amplitude comprised between 100 and $117 \mathrm{~V} / \mathrm{cm}$ and a potential level of between 200 and $250 \mathrm{~V}$ for the successful ignition waves (i.e. $V_{A}$ equal or higher than $-400 \mathrm{~V}$ ). All these waves share a nearly identical slope in this period ( 78 to $98 \mathrm{MVcm}^{-1} \mathrm{~s}^{-1}$ ) and present a relatively small difference in the peak potential despite the large range of applied voltage levels. This non linear behaviour occurs as a consequence of an increase of the spread of the ionization wave front at higher applied voltages. This phenomenon can be understood by considering that a higher $V_{A}$ amplitude leads to a higher $d V / d t$ value during the voltage rise time, thus leading to faster electrons and to a stronger electron flux being emitted from the cathode. This in turn causes an increased ionisation rate in the vicinity of the electrode, with ionisation reactions occurring farther ahead, and a faster charging rate of the inner wall surface of the lamp. An observation of the optical emission and of the E field profile (see the FWHM values in table 3, section 5.2) of the wave indeed confirms the spread of the pre breakdown wave front at higher $V_{A}$ values.

The pre-breakdown waves associated with failed ignitions at $-300 \mathrm{~V}$ or lower show a lower slope in the increase of $\mathrm{E}$ field strength $\left(31 \mathrm{MVcm}^{-1} \mathrm{~s}^{-1}\right)$ during the voltage rise time than for the waves at higher $V_{A}$ values whose slope are more than twice as high. The peaks of E field and of potential are also reached at a later stage, typically $4.5 \mu$ s instead of $1.5 \mu$ s and present much lower values of $-127.3 \mathrm{~V}$ and $80.3 \mathrm{~V} / \mathrm{cm}$ at $V_{A}=-300 \mathrm{~V}$ and $-155.3 \mathrm{~V}$ and $79.2 \mathrm{~V} / \mathrm{cm}$ at $V_{A}=-200 \mathrm{~V}$. The E field amplitude is nearly the same for both pre-breakdown waves despite a $22 \%$ difference in wave front potential. This arises as the wave front length is longer at $-300 \mathrm{~V}(1.9 \mathrm{~cm})$ than at $-200 \mathrm{~V}(1.6 \mathrm{~cm})$. 


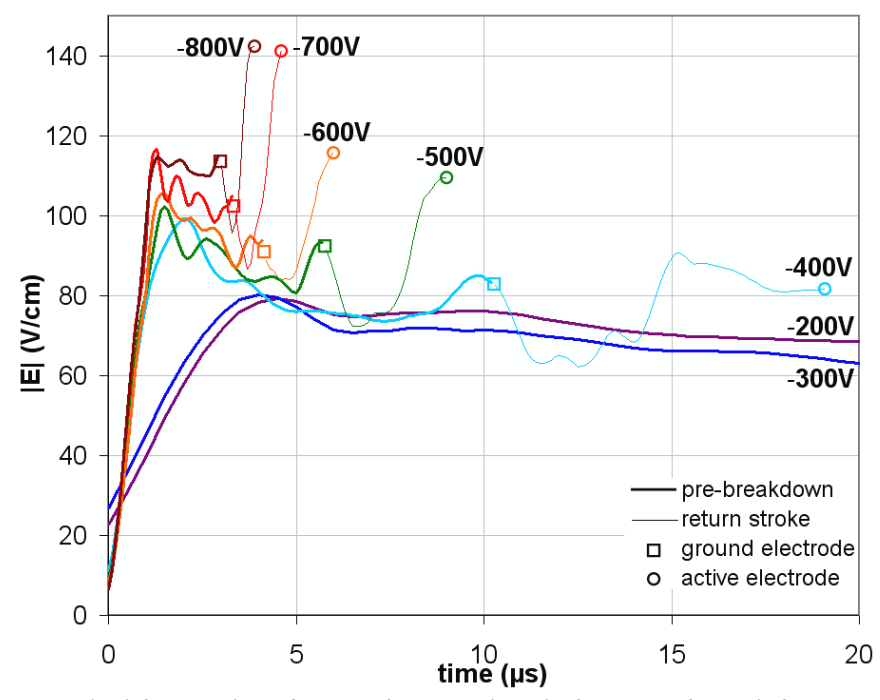

Figure 16: Electric field amplitude at the peak of the pre-breakdown wave and of the return stroke in 3 Torr argon as a function of time and of the applied voltage amplitude. Ignitions below $-400 \mathrm{~V}$ are unsuccessful.

The $\mathrm{E}$ field strength of the pre-breakdown wave front gradually decreases during its propagation. For successful ignitions this evolution goes from 99.2 to $73.8 \mathrm{~V} / \mathrm{cm}$ at $-400 \mathrm{~V}$, and from 114.7 to $110.0 \mathrm{~V} / \mathrm{cm}$ at $-800 \mathrm{~V}$, indicating that the relative decrease of $\mathrm{E}$ field amplitude during propagation is higher at lower applied voltages $(-4.1 \%$ at $-800 \mathrm{~V}$ versus $-25.6 \%$ at $-400 \mathrm{~V})$. This trend has two causes, firstly there is a decrease of the electric potential at the wave front and secondly the wave front spreads during its propagation. For successful ignitions the wave front potential evolves from -243.4 to $204.0 \mathrm{~V}(-16.2 \%)$ at $V_{A}=-800 \mathrm{~V}$, and from 203.9 to $128.0 \mathrm{~V}(-37.2 \%)$ at $V_{A}=-800 \mathrm{~V}$, showing also that the relative decrease of potential is higher at lower applied voltages. This potential decrease finds its origin in the electron current which flows in the wake of the ionisation wave, from the active electrode to the wave front. As the wave progresses the ohmic impedance of this wake increases and for a given charging current the voltage drop across this ionised channel steadily increases in time and space, thus leading to the gradual decrease of the wave front potential.

The minimum $E$ field strength reached by a pre-breakdown wave propagating in a successfully igniting lamp is $75 \mathrm{~V} / \mathrm{cm}$ at $-400 \mathrm{~V}$, close to the minimum field (about $72 \mathrm{~V} / \mathrm{cm}$ ) needed to sustain the propagation of the ionisation wave (see the evolution of the wave for failed ignitions, figure 16). During the pre-breakdown phase an oscillation of the wave front potential and E field is observed. This ripple cannot be related to fluctuations in the applied unipolar voltage waveform, and the fact that the period of this oscillations decreases at higher $V_{A}$ values point toward a Frank and Hertz effect [44]. 


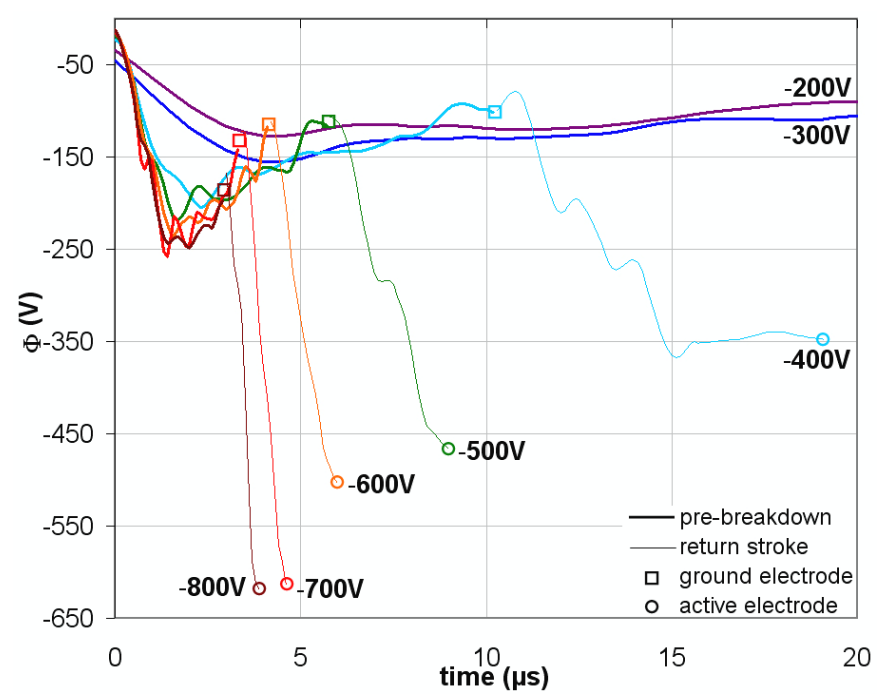

Figure 17: Electrostatic potential at the peak of the pre-breakdown wave and of the return stroke in 3 Torr argon as a function of time and of the applied voltage amplitude. Ignitions below $-400 \mathrm{~V}$ are unsuccessful.

An increase of the E field strength of the pre-breakdown wave front occurs at the end of its propagation. This phenomenon cannot be explained in terms of wave front potential since it continuously decreases, so the E field enhancement finds its origin from the closing of the gap between the pre-breakdown wave and the ground electrode. This increase of local E field strength (from 110 to $115 \mathrm{~V} / \mathrm{cm}$ at $-800 \mathrm{~V}$, and from 73 to $84.9 \mathrm{~V} / \mathrm{cm}$ at $-400 \mathrm{~V}$ ) is a source of increased ionisation in the region of the ground electrode and leads to the formation of the return stroke. This second wave propagates back toward the active electrode with a wave front potential that increases nearly linearly in time and reaches a final value close to that of the active electrode. The difference in potential varies from $50 \mathrm{~V}$ at $-400 \mathrm{~V}$ to $150 \mathrm{~V}$ at $-800 \mathrm{~V}$.

From the start of its formation at the ground electrode, the return stroke has an E field strength which forms a dip for a period that ranges from $4.3 \mu \mathrm{s}$ at $-400 \mathrm{~V}$ to $0.6 \mu \mathrm{s}$ at $-800 \mathrm{~V}$ (figure 16). This corresponds to a phase when charges build up in the gap between the pre-breakdown wave front and the ground electrode. Once a sufficient amount of charge is accumulated there, the return stroke starts its propagation from this region and its wave front $\mathrm{E}$ field strength increases steadily to a maximum ranging from $90.6 \mathrm{~V} / \mathrm{cm}$ at $-400 \mathrm{~V}$ to $142 \mathrm{~V} / \mathrm{cm}$ at $-800 \mathrm{~V}$, at the end of its propagation .

\subsection{Current and net charge evolutions}

The measured total lamp displacement current during the propagation of the pre-breakdown wave is found to flow from the Faraday cage to the lamp (figure 18) leading to the negative charging of the lamp (figure 19). The evolution in time of this current has two origins: the charging of the lamp by the moving pre-breakdown wave front and the evolution in time of the electrostatic potential in the wake of the ionisation wave (see potential map in figure 10). For all cases (except at $-200 \mathrm{~V}$ ) the current peak occurs at $2 \mu \mathrm{s}$, at the end of the voltage rise at the active electrode. This peak originates both from the rise of the active electrode voltage and from the build up of charges in the vicinity of this electrode due to ionisation processes. The peak of displacement current ranges from $-400 \mu \mathrm{A}$ at $-400 \mathrm{~V}$ to -1600 $\mu \mathrm{A}$ at $-800 \mathrm{~V}$ and is found to be nearly proportional to the applied voltage amplitude $V_{A}$. The influence on the current waveform of the rise of $V_{A}$ during the first $2 \mu$ s extends in fact to $3 \mu \mathrm{s}$ as a result of the formation of the cathode sheath (section 3.1). 


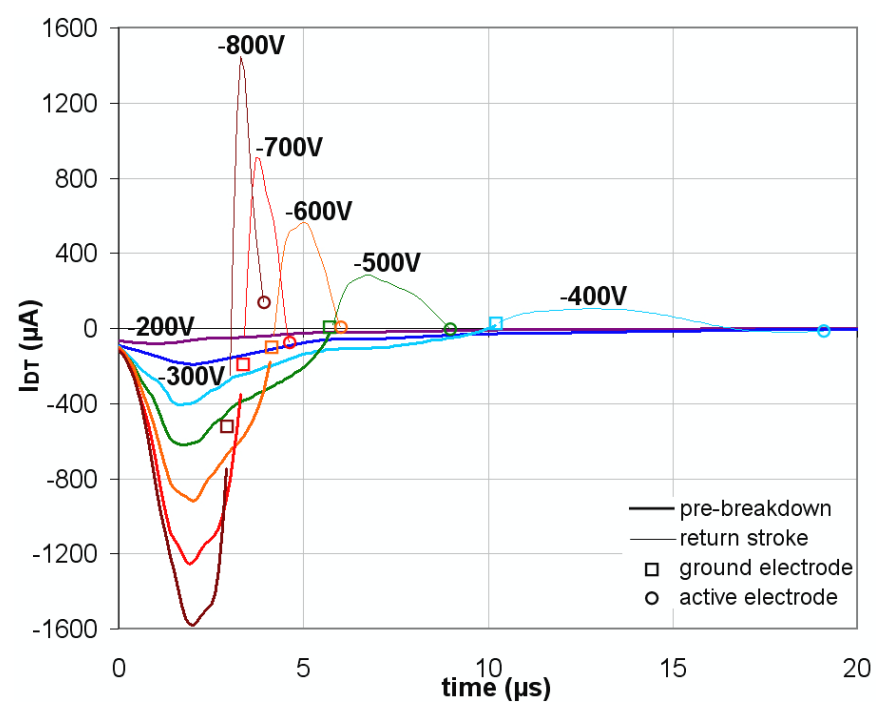

Figure 18: Total displacement current flowing from the argon lamp as a function of time and of the applied voltage amplitude. The current is positive from the lamp to the Faraday cage. Ignitions below -400 V are unsuccessful.

After $t=2 \mu$ s the displacement current continuously decreases in time until the pre-breakdown wave reaches the ground electrode, at which point the current reaches zero. During this period the lamp charges negatively with a decreasing rate related to the evolution of the displacement current. The observed decreasing displacement current indicates that the electron current flowing from the active electrode to the wave front decreases also in time during the wave propagation. This is consistent with an increasing ohmic impedance of the wake leading to a decrease in the wave front potential, E field strength and ionisation rate. The increase of wave front $\mathrm{E}$ field strength observed at the end of the wave propagation in figure 16 does not lead to a peak of displacement current because during this event the charges flow directly from the pre-breakdown wave front to the ground electrode, and not toward the Faraday cage. The displacement current thus reaches zero and the lamp charge reaches a maximum point which increases for higher applied voltages $(-1.74 \mathrm{nC}$ at $-400 \mathrm{~V}$ to $-2.94 \mathrm{nC}$ at $-800 \mathrm{~V})$ at the end of the pre-breakdown wave propagation.

The return stroke propagation has the effect of decreasing the net lamp charge, resulting in the reversal of the displacement current to positive values (figure 18). The value of total lamp net charge reached at the end of the propagation of this wave is very close to that corresponding to a lamp being nearly charged to half the active electrode potential, consistent with the nearly constant potential gradient observed in figure 10 at $9 \mu$ s leading to a constant axial $\mathrm{E}$ field across the lamp (figure 11). The small observed difference arises from the potential drop across the cathode sheath.

It is found that the maximum lamp net charge $Q_{T m}$ reached at the end of the pre-breakdown wave front propagation is not exactly proportional to $V_{A}$ and does not corresponds to a full charging of the lamp to the active electrode potential. An analysis is done by estimating the equivalent capacitance value $C_{E}$ fully charged up to $V_{A}$ according to expression (13):

$$
C_{E}=\frac{Q_{T m}}{V_{A}}
$$




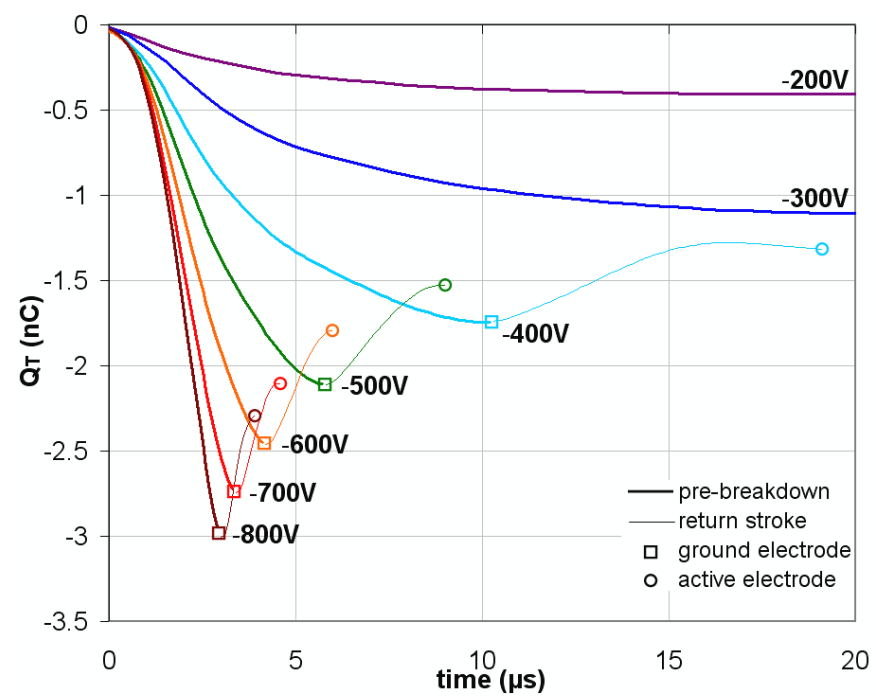

Figure 19: Total net charge of the 3 Torr argon lamp as a function of time and of the applied voltage amplitude. Ignitions below $-400 \mathrm{~V}$ are unsuccessful.

The values of $Q_{T m}$ and $C_{E}$ are given in table 1 as a function of $V_{A}$. The value of $C_{E}$ is compared to that of the total lamp capacitance $C_{T}$ over the $150 \mathrm{~mm}$-long region scanned by the electrostatic probe in order to estimate the charged capacity of the lamp. Likewise, we estimate the equivalent length $L_{E}$ of lamp charged up to $V_{A}$ assuming that the wake of the pre-breakdown wave has a constant potential (i.e. step profile of the wave).

$$
L_{E}=\frac{C_{E}}{C_{l}}=\frac{Q_{T m}}{V_{A} \cdot C_{l}}
$$

Where $C_{l}$ is the lamp linear capacitance (equal to $37.8 \mathrm{fF} / \mathrm{mm}$ ). The hypothesis underlying (14) is naturally not true in the present case (figure 10 and 20) but it still enables the understanding of the capacitive behaviour of the lamp and of its charging dynamics during successful and failed ignitions.

Table 1: Measured total lamp net charge $Q_{T m}$ at the end of the pre-breakdown wave propagation and calculated equivalent charged capacitance $C_{E}$ and length $L_{E}$ as a function of the applied voltage level $V_{A}$.

\begin{tabular}{|c|c|c|c|}
\hline $\boldsymbol{V}_{\boldsymbol{A}}(\mathbf{V})$ & $\boldsymbol{Q}_{\boldsymbol{T}}(\mathbf{n C})$ & $\boldsymbol{C}_{E}(\mathbf{p F})\left[\% \boldsymbol{C}_{\boldsymbol{T}}\right]$ & $\boldsymbol{L}_{E}(\mathbf{m m})$ \\
\hline-200 & -0.408 & $2.040[36.0]$ & 53.9 \\
\hline-300 & -1.11 & $3.700[65.3]$ & 97.9 \\
\hline-400 & -1.743 & $4.358[76.9]$ & 115.3 \\
-500 & -2.108 & $4.216[74.4]$ & 111.5 \\
-600 & -2.463 & $4.105[72.4]$ & 108.6 \\
\hline-700 & -2.747 & $3.924[69.2]$ & 103.8 \\
\hline-800 & -3.003 & $3.754[66.2]$ & 99.3 \\
\hline
\end{tabular}

The results presented in table 1 concern both successful $\left(V_{A}<-300 \mathrm{~V}\right)$ and failed ignitions $\left(V_{A}>-400 \mathrm{~V}\right)$ and show that the lamp is indeed partially charged at the end of the first wave propagation. This can be understood by the fact that there exists a potential gradient in the wake of the pre-breakdown wave (figure 10) which leads to a wave front potential and an average wave potential that are below that of the active electrode. The charging percentage has a maximum at $76.9 \%$ at a $V_{A}$ value of $-400 \mathrm{~V}$, near 
the lamp static ignition voltage, and decreases at higher applied voltage amplitudes. This trend is not caused by the potential gradient in the wake of the pre-breakdown wave since the relative potential difference between the active electrode and the wave front actually decreases at higher applied voltages (section 3.3), which should normally lead to an increase of $C_{E}$, not its decrease. The observed trends thus have some other origins found in the analysis of the trend of $L_{E}$.

The equivalent length of ionisation wave also has a maximum at $-400 \mathrm{~V}(115 \mathrm{~mm})$ and decreases for higher voltages, which seems to indicate that the bridging between the pre-breakdown wave and the ground electrode occurs at longer separation lengths for higher $V_{A}$ values because of the higher electric field strength in the front of the waves (section 3.3). Likewise, at low applied voltages the lamp needs to be relatively more fully charged for a successful ignition to occur because the low $\mathrm{E}$ field strength in its pre-breakdown wave front makes the bridging with the ground electrode more difficult, the ionisation processes being more localized. This thus requires the wave to propagate further toward this electrode for the bridging to effectively occur. The calculation of the average electric field strength in the wave-electrode gap (wave front potential divided by the gap length) reveals that the effective bridging occurs at a nearly constant field amplitude of between 32.0 and 36.7 $\mathrm{V} / \mathrm{cm}$ for all $V_{A}$ values above $-300 \mathrm{~V}$. It can therefore be concluded that the effective gap length leading to the connection of the pre-breakdown wave to the ground electrode is set by a minimum average field strength requirement. This therefore explains that at higher $V_{A}$ value this gap length increases and the effective capacitance decreases. It is found that the equivalent capacitance and equivalent length values for successful ignitions follow a linear relationship with $V_{A}$, which for $C_{E}$ yields:

$$
C_{E}=C_{T}\left(2.64 \cdot 10^{-4} \cdot V_{A}+0.88\right)
$$

In the extreme case of low applied voltages where the lamp fails to ignite, the trend of the charged capacity is inversed, lower applied voltages will actually lead to a lower equivalent length and capacity. This evolution is due to the fact that the pre-breakdown wave, responsible for charging the lamp, wanes out before bridging with the ground electrode. It results that $C_{E}$ and $L_{E}$ corresponds to the point where the pre-breakdown wave extinguishes. This observation and the trends presented in table 1 lead to the third condition for successful ignition: At the static ignition voltage a sufficient amount of charge need to be provided in order to charge the lamp to a minimum level enabling the full propagation of the pre-breakdown wave and its bridging with the ground electrode. Data in table 1 seem to indicate that this level is at around $80 \%$ of the lamp total capacitance, which corresponds to a $L_{E}$ value of $116 \mathrm{~mm}$, or a gap length between the idealized pre-breakdown wave and the ground electrode of $29 \mathrm{~mm}$, nearly equal to the inner radius of the Faraday cage $(27 \mathrm{~mm})$. This seem to indicate that the minimum gap length to be bridged by the wave of the lowest front $\mathrm{E}$ field strength (thus at an applied voltage equal to the static breakdown voltage) is equal to the distance from the lamp axis to the nearest ground surface, at which point the axial and radial components of the E field become equal.

\subsection{Failed negative ignition and memory effect}

In the data presented in section 3.2 it is observed that the pre-breakdown wave does not reach the ground electrode below a certain voltage threshold which corresponds to the static ignition voltage. This situation not only leads to ignition failure, but also to a memory effect which negatively affects the subsequent breakdown processes when a voltage pulse of same polarity and amplitude is applied again. The map of optical emission occurring in the lamp at $-300 \mathrm{~V}$ (figure 20) shows that the prebreakdown wave starts from the active electrode, propagates with the same trends as observed in 
section 3.1, with its speed and light intensity decreasing in time and space, and the wave finally extinguishes at $15 \mu \mathrm{s}$ and no other light emission is observed in the lamp in the next $75 \mu \mathrm{s}$.

The measured electrostatic potential from the capacitive probe (figure 20) shows a potential gradient developing during the propagation of this pre-breakdown wave which is similar in shape to that measured for a successful ignition at $-500 \mathrm{~V}$ (figure 10). This gradient leads to the formation of a local region of high E field strength (figure 21) which drives the pre-breakdown wave. The decrease in the light emission associated with this wave is consistent with the gradual decrease of the E field amplitude whose associated vector angle remains between $-110^{\circ}$ and $-120^{\circ}$ (i.e. stronger axial component) during the entire wave propagation. These observations are similar to those made in section 3.1 for successful ignitions and as remarked in this section, the dip in the supply voltage observed at $5 \mu \mathrm{s}$ is an undershoot from the power supply and is not caused by the igniting lamp.
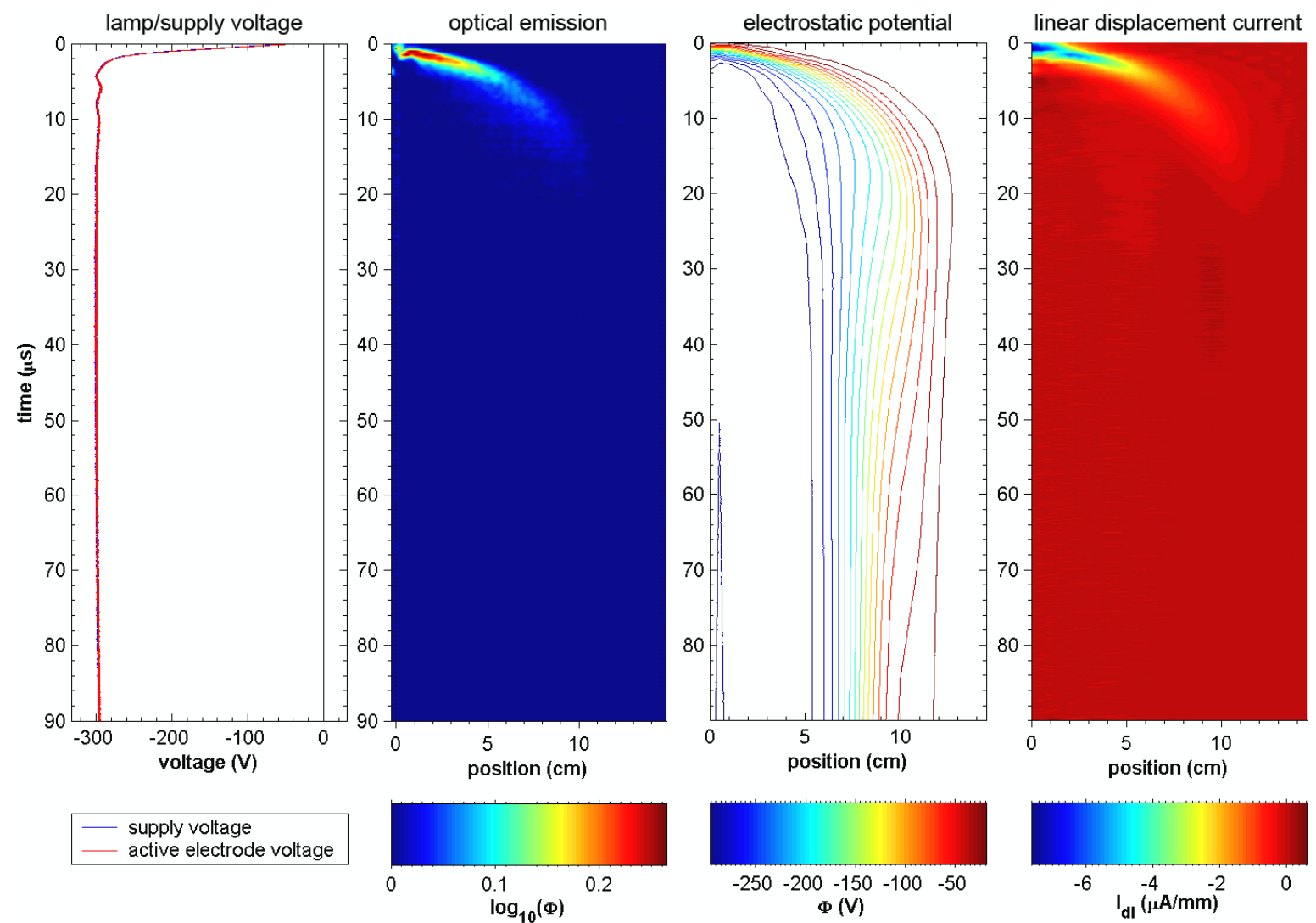

Figure 20: Space-time diagrams of the evolution of the measured light emission, of the net electrostatic potential and of the calculated linear displacement current (positive from the lamp to the Faraday cage) associated with failed breakdown phenomena occurring in 3 Torr argon at $-300 \mathrm{~V}$. The active and the ground electrodes are located at 0 and $14.5 \mathrm{~cm}$, respectively.

In the present situation the potential gradient develops up to $12.5 \mathrm{~cm}$ and remains after the point when the ionisation wave has stopped its propagation $(15 \mu \mathrm{s})$ which is evidence of remaining charges left in the lamp. A displacement current flowing from the Faraday cage to the lamp is also associated with the propagation of this ionisation wave, which is coherent with the negative charging of the lamp. However, no discernible discharging current is seen in figure 20 after the extinction of the wave, confirming the earlier observation that the lamp has retained its electrostatic charge. This phenomenon 
is also observed from the total lamp net charge data of figure 19 in the previous section. What is not shown there is that at $-300 \mathrm{~V}$ the $Q_{T}$ value increases up to $-1.11 \mathrm{nC}$ at $22.5 \mu \mathrm{s}$ and then decreases to $0.98 \mathrm{nC}$ at $94 \mu \mathrm{s}$. This decrease of the total lamp net charge after $22.5 \mu$ s arises as a consequence of a re-organization of charges in the wave front region $(7$ to $12 \mathrm{~cm}$ ) and close to the active electrode (1 to $2 \mathrm{~cm}$ ). This firstly leads to a retreat of the potential gradient back toward the active electrode, displacing the region of $\mathrm{E}$ field peak strength from $12 \mathrm{~cm}$, where the wave stopped, to $8 \mathrm{~cm}$ (figure 21). In this process a slightly positive displacement current occurs between 20 and $80 \mu$ s and 8 and $11 \mathrm{~cm}$. The reconfiguration of the potential gradient has an impact on the E field which shows an increased in its peak strength from 60 to about $100 \mathrm{~V} / \mathrm{cm}$ in the wave front region. This evolution is most likely caused by ion diffusion in the ionised medium. The E field strength in the wave front at $20 \mu$ s before the charges re-organise is at around $60 \mathrm{~V} / \mathrm{cm}$ and the corresponding ion mobility is about $125 \mathrm{~cm}^{2} \mathrm{~V}^{-1} \mathrm{~s}^{-1}$, leading to an ion drift velocity of $7500 \mathrm{~cm} / \mathrm{s}$. Considering the lamp inner radius of 0.5 $\mathrm{cm}$, the drift time of ions from the axis to the wall is thus $67 \mu \mathrm{s}$, the same order of magnitude as the observed charge re-organization time, thus confirming the role of the ions in this process.
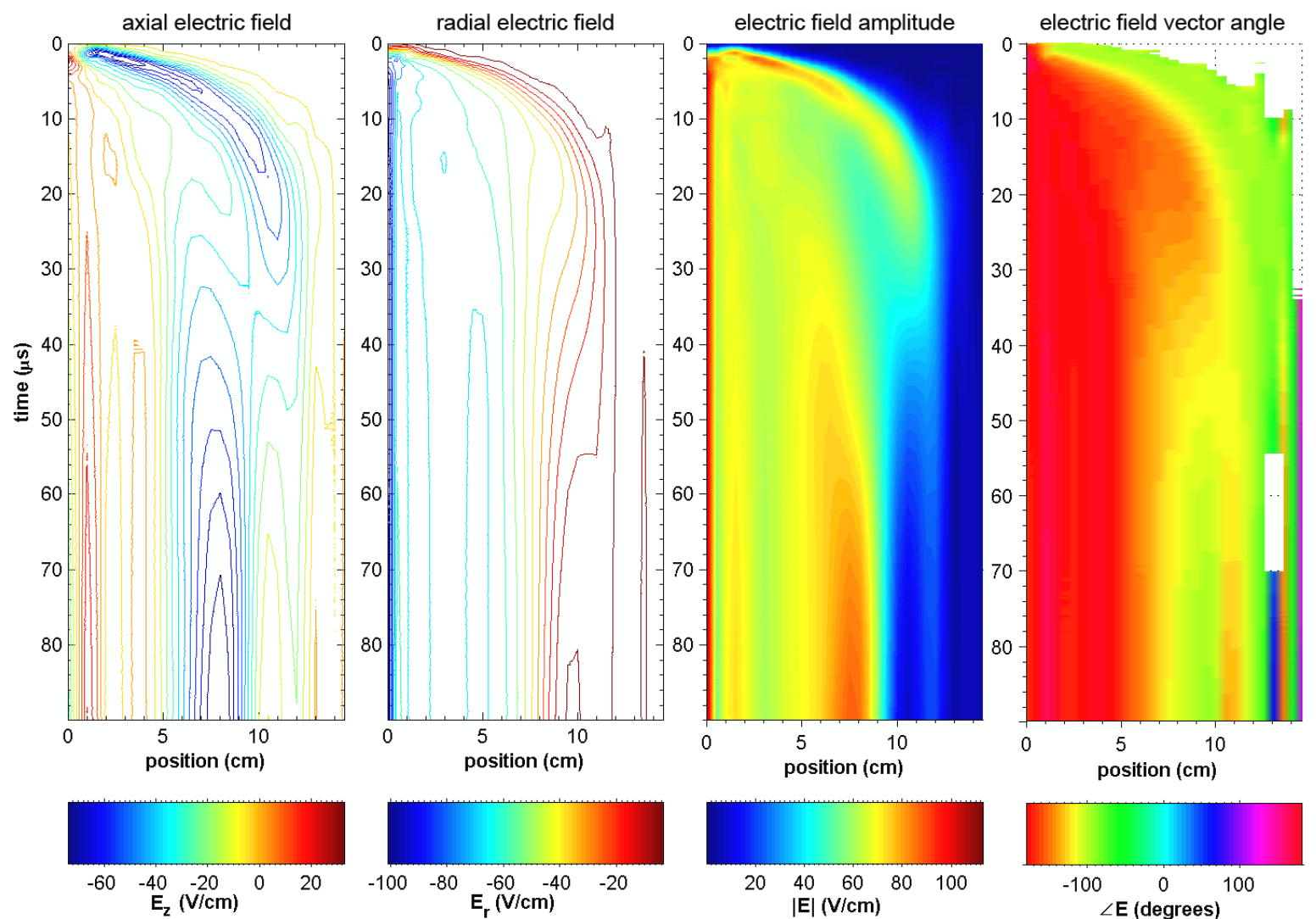

Figure 21: Space-time diagrams of the evolution of the axial electric field (positive from the active to the ground electrode), of the radial electric field (positive from the lamp to the Faraday cage), of the electric field amplitude and vector angle (null when oriented from the lamp wall to the Faraday cage, and oriented from the active to the ground electrode at $+90^{\circ}$ ) associated with failed breakdown phenomena at $-300 \mathrm{~V}$ in 3 Torr argon. The active and the ground electrodes are located at 0 and $14.5 \mathrm{~cm}$, respectively.

Secondly, the other impact of the re-organization of charges is the development of a second region of strong E field strength at $1.5 \mathrm{~cm}$ from the active electrode, leading to an axial field reversal most 
probably caused by an excess of electrons in the region from 5 to $8 \mathrm{~cm}$. This situation eventually becomes stable and yields two durable regions of high $\mathrm{E}$ field strength from $50 \mu \mathrm{s}$ on. The first peak at $1.5 \mathrm{~cm}$ has a field amplitude of $74 \mathrm{~V} / \mathrm{cm}$ and an associated field vector angle of $+160^{\circ}$ resulting from the radial nature of the field in this region. The second peak at $7.5 \mathrm{~cm}$ has a higher field strength $(88$ $\mathrm{V} / \mathrm{cm})$ and an essentially axial field vector $\left(-110^{\circ}\right)$. The lamp remains strongly polarized up to $7 \mathrm{~cm}$, and these negative wall charges then cause a lowering of the electric field strength in the vicinity of the active electrode by a screening effect when other negative voltages pulses are applied to the lamp. This leads to the ignition difficulties, or quenching, which are encountered upon the application of the next voltage pulse when no resetting pulses are applied to the lamp.

The phenomena responsible for the observed charge redistribution are likely to develop as follows. There is a point in time when the electron current flow in the wake of the pre-breakdown wave can no longer feed the wave front, which eventually extinguishes. However, the local E field strength there is still high enough to drive the electron avalanche further and thus lead to a charge separation which is no longer compensated by the electron flux from the active electrode. In figure 20 the displacement current falls nearly to zero from 10 to $15 \mu \mathrm{s}$, but in figure 21 the electric field profiles continue to evolve until $30 \mu \mathrm{s}$. After $20 \mu \mathrm{s}$ the displacement current has become zero and the progression of the wave is solely driven by the local $\mathrm{E}$ field which leads to a negatively charged front resulting from the moving electron avalanche, and to a positive tail of free ions. The ions will then diffuse along the internal $\mathrm{E}$ field lines to recombine at the wall with electrons. There is no measured displacement current associated with this process since it does not lead to any net change of charge in the lamp.

\subsection{Summary of negative ignition processes}

Three main phases associated with a successful ignition above $-300 \mathrm{~V}$ are observed upon the application of a negative voltage pulse to the active electrode: first, a pre-breakdown wave is initiated at the active electrode and propagates in a neutral lamp toward the ground electrode, located on the opposite side of the lamp. Once the wave has reached its destination a second wave, the return stroke, propagates back toward the active electrode and draws a luminous channel in its wake. In the third phase the homogeneous plasma channel which now exists between the electrodes has a light intensity which increases in time until the lamp reaches its steady state regime.

The initiation of the pre-breakdown wave happens in the active electrode region upon the acceleration of thermally-emitted electrons toward the wall, which causes Townsend-like ionisation to occur. This ionization front displaces itself ahead of the active electrode as a result of lamp charging which causes the formation of the cathode sheath, and displaces the region of high electric field. The wave is associated with a displacement current which flows from the Faraday cage to the lamp as a result of the negative charging of the lamp during the wave propagation. This charging attains a peak level when the wave reaches the ground electrode. The pre-breakdown wave has a velocity comprised between 23 and $65 \mathrm{~km} / \mathrm{s}$, which is proportional to the applied voltage and decreases in time and space as a result of the gradual decrease of its wave front potential and electric field. This electric field is essentially axial in nature due to the presence of a strong potential gradient in the lamp, and its decrease in time leads to a decrease of intensity of its associated light emission and of its displacement current as a result of a decrease of the local ionisation rate and of the current that flows from the active electrode to the wave front.

The displacement of the pre-breakdown wave toward the ground electrode leads to an increase of the local electric field in front of this electrode. This results in the electrical breakdown of the wave front-electrode gap which occurs at a constant average electric field strength (between 32.0 and $36.7 \mathrm{~V} / \mathrm{cm}$ ) for all applied voltages above $-300 \mathrm{~V}$. This phase coincides with the closing of the 
electrical circuit, the formation of the anode sheath and the start of the second wave, the return stroke. This wave propagates back toward the active electrode leading to the partial discharging of the lamp and to a homogenisation of the potential gradient between the electrodes. This wave presents similar propagation features (light emission, evolution of position in time) as those of the pre-breakdown wave, but has a higher E field strength in its wave front and has a sharply decreasing potential in space.

It is observed that the full propagation of the pre-breakdown wave is the main requirement for the development of the next processes and the successful ignition of the lamp. This sets the condition not only for a minimum applied voltage of $-400 \mathrm{~V}$ in the present experiments (which sets a minimum wave front $\mathrm{E}$ field strength of $72 \mathrm{~V} / \mathrm{cm}$ ), but also for a minimum duration of the applied voltage pulse of $10.2 \mu$ s (to enable the full propagation of the wave) and a minimum amount of charges to be provided for the electrostatic charging of the lamp $(-1.74 \mathrm{nC}$ at $-400 \mathrm{~V})$. In cases one of these requirements are not met, then the pre-breakdown wave will still start from the active electrode, it will propagate in the lamp in a similar manner as at higher voltages, but it will extinguish without connecting with the ground electrode. In this process a certain amount of negative charges will remain in the lamp leading to a memory effect. This will result in the lowering of the local electric field surrounding the active electrode once other voltage pulses are applied to the lamp, thereby quenching the local Townsend avalanche which is needed for the creation of the pre-breakdown wave.

\section{Ignition processes at positive potentials}

In the positive ignition experiments the amplitude of the voltage pulse applied to the active electrode ranges from $+400 \mathrm{~V}$ to $+700 \mathrm{~V}$ with measurements done with $50 \mathrm{~V}$ and $100 \mathrm{~V}$ steps. The time resolution of the processed optical and electrostatic data is $500 \mathrm{~ns}$ for +400 and $+450 \mathrm{~V}$ and is $100 \mathrm{~ns}$ for higher voltage amplitudes.

\subsection{Overview of phenomena}

The space-time diagrams of the measured light emission, of the electrostatic potential and of the calculated linear displacement currents are presented in figure 22 in the case of a successful ignition at $+500 \mathrm{~V}$. The optical recording of the light emission associated with the positive ignition (figure 22) presents the three ignition phases that were described in section 3.1. Upon the application of the positive potential to the active electrode, a luminous channel associated with the first ionization wave starts at this electrode and propagates toward the opposite (ground) electrode. Once this prebreakdown wave reaches its destination a second luminous channel propagates back toward the active electrode and draws a homogeneous plasma channel in its wake, whose light intensity increases in time until the lamp reaches a steady state regime.

The first phenomenon to occur in the lamp during the voltage rise phase $(0-2 \mu \mathrm{s})$ is not clearly identifiable in the optical recordings, but is apparent in the displacement current map of figure 22 between 1 and $2 \mu \mathrm{s}$ and up to $3 \mathrm{~cm}$ ahead of the active electrode. This phenomenon is the neutralization of the negative space charge which was created by the negative pre-ionization pulse applied prior to the main positive pulse (section 2.4). The pre-breakdown wave which starts its propagation during this phase does not seem to be perturbed by this neutralization process since its optical features and profile do not differ from those at later times in its propagation. This wave has a very narrow light emission profile, measured to be $4.5 \mathrm{~mm}$ wide at $+500 \mathrm{~V}$, and propagates in a linear fashion between the two electrodes. The transit time of this wave is relatively long, $12.5 \mu$ s versus $5.5 \mu$ s for the negative pre-breakdown wave. 

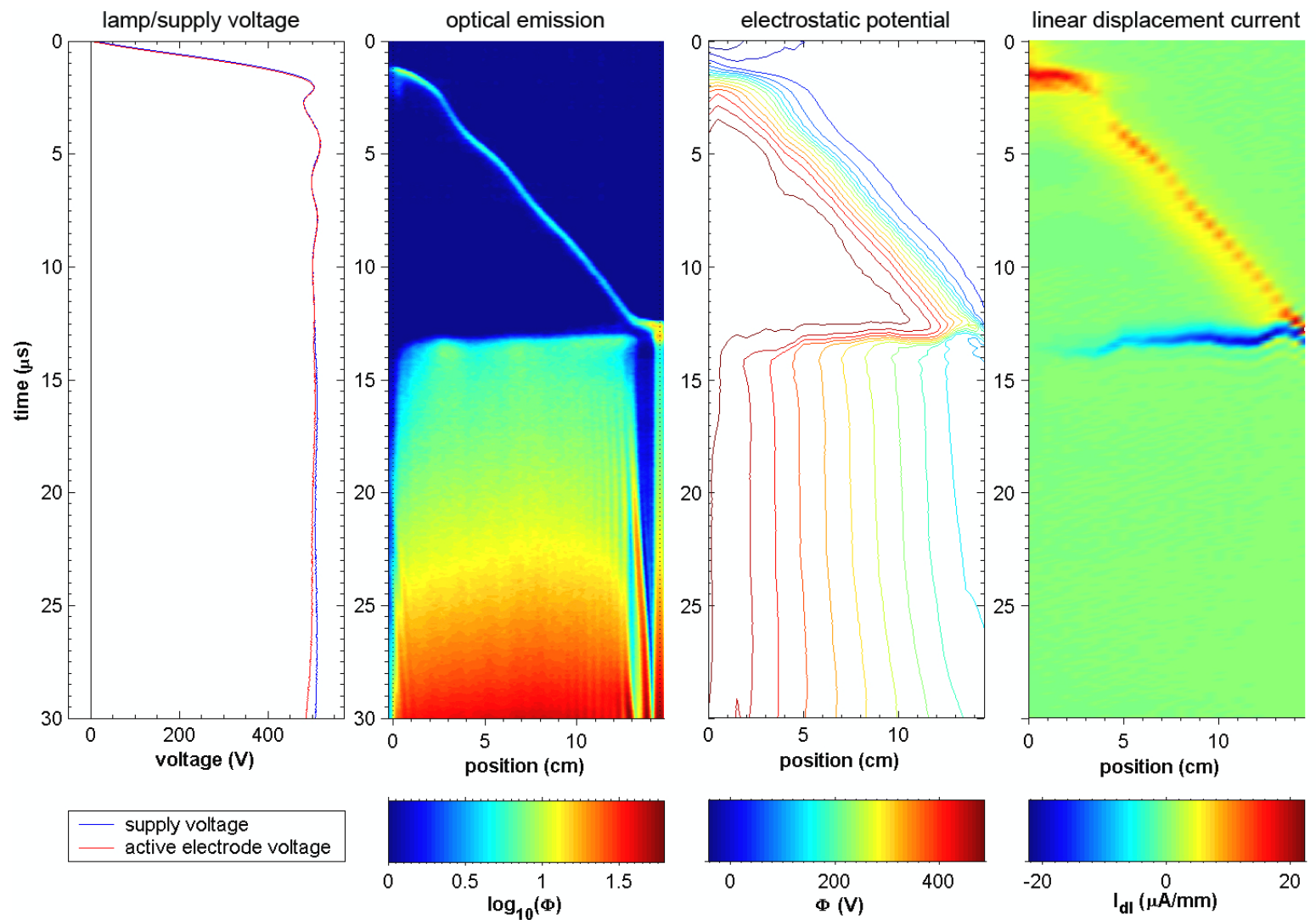

Figure 22: Space-time diagrams of the evolution of the measured light emission and net electrostatic potential and of the calculated linear displacement current (positive from the lamp to the Faraday cage) associated with breakdown phenomena occurring in 3 Torr argon at $+500 \mathrm{~V}$. The active and the ground electrodes are located at 0 and $14.5 \mathrm{~cm}$, respectively.

During its propagation the positive pre-breakdown wave does not spread over time and space and is associated with a local region of strong potential gradient whose profile is broader $(2.45 \mathrm{~cm}$ full-width at half maximum, table 4 in section 5.3) but also remains constant during the wave propagation. This potential gradient leads to a wave front having a strong and localized axial E field (figure 23) whose amplitude is higher than the radial component, leading to a constant field vector angle of only $+100^{\circ}$ despite the close proximity of the grounded Faraday cage. The narrow potential gradient in the wave front has therefore a larger impact on the E field direction than the Faraday cage. The axial E field presents also a narrow profile whose constant feature in time and space and peak location coincides with those of the associated light emission. The radial E field component presents a gradient in the wave front region consistent with the potential profile of the wave. Both field components have a profile in the wave front which has a constant shape during the wave propagation.

As opposed to the negative breakdown case (section 3.1), the potential gradient in the wake of the positive pre-breakdown wave is nearly null. This results either from a very low electron current flowing from the wave front to the active electrode, or from a high electrical conductivity in the ionised medium of the wake. The linear displacement current map (figure 22) features a narrow current profile that matches that of the optical emission and of the E field map (figure 23). The profile of the displacement current wave being smaller than the $5 \mathrm{~mm}$ of the RC probe spatial resolution results in the stepped pattern displayed in figure 22. Moreover, this current wave presents a peak value which is 
higher than for negative ignitions. This is an indication that the electron current flux in the lamp during ignition at positive potential is higher, and it logically results that the weak potential gradient in the pre-breakdown wake is caused by a high electrical conductivity. The polarity of the displacement current is also reversed compared to the negative ignition case, which is consistent with the charging of the lamp at a positive potential.
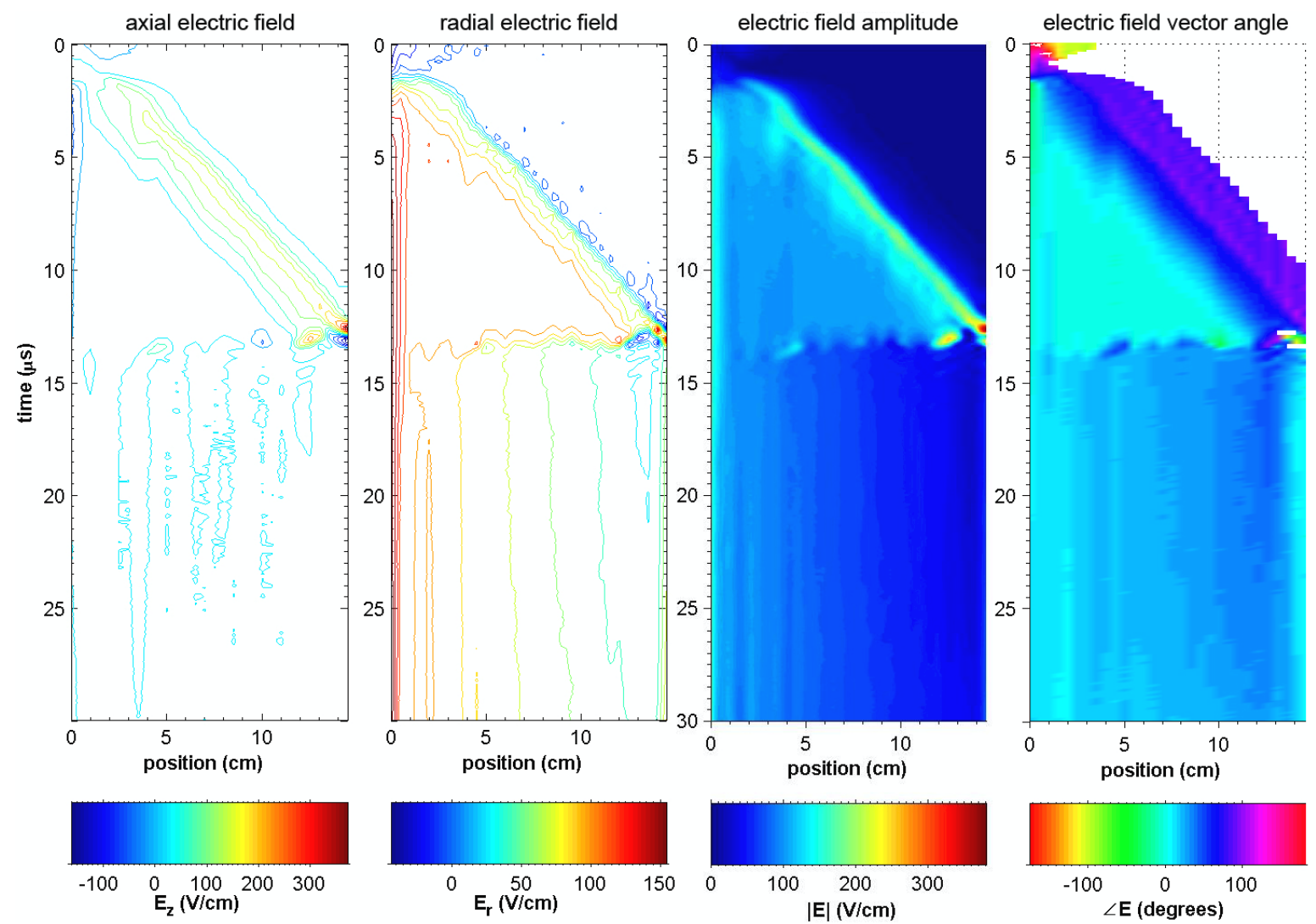

Figure 23: Space-time diagrams of the evolution of the axial electric field (positive from the active to the ground electrode), of the radial electric field (positive from the lamp to the Faraday cage), of the electric field amplitude and vector angle (null when oriented from the lamp wall to the Faraday cage, and oriented from the active to the ground electrode at $+90^{\circ}$ ) associated with breakdown phenomena occurring in 3 Torr argon at $+500 \mathrm{~V}$. The active and the ground electrodes are located at 0 and $14.5 \mathrm{~cm}$, respectively.

The form of the second ionization wave, the return stroke, differs markedly from the observations at negative polarity in the fact that the positive wave presents a much faster propagation, bridging the two electrodes in less than $1 \mu \mathrm{s}$, versus $2.5 \mu \mathrm{s}$ at $-500 \mathrm{~V}$. The passage of the return stroke causes a redistribution of potential that leads to a nearly constant gradient between the electrodes (figure 22). It results that the lamp gradually discharges in this process, as evidenced by the negative displacement current associated with this wave. The return stroke has a nearly zero axial electric field amplitude which indicates that this return wave is not likely to present an increase of ionization rate in its wave front. The angle of its $\mathrm{E}$ field vector at the wave front fluctuates between 0 and $+90^{\circ}$ during propagation from causes that are not yet understood. A potential cause may be the spatial variation of the charge density in the ionised medium of the pre-breakdown wake. 
The formation of the electrode sheaths are also observed during the $30 \mu \mathrm{s}$ of the recordings. The first sheath starts to form at $1.5 \mu \mathrm{s}$ in front of the active electrode (anode). The first feature of the anode sheath is a reversed axial field, located at between 0 and $1 \mathrm{~cm}$, which decreases in strength from 5 to $13 \mu \mathrm{s}$ as a result of charge build up. A localized gradient of strong radial E field develops at the same location and time period, and then remains in front of the active electrode for the rest of the measurements. The cathode sheath is formed when the pre-breakdown wave reaches the ground electrode at $12.5 \mu \mathrm{s}$, resulting first in a burst of reversed axial $\mathrm{E}$ field in front of the cathode which lasts $0.5 \mu \mathrm{s}$. From this point a gradient of radial E field develops immediately between 14.0 and 14.5 $\mathrm{cm}$ and remains for the rest of the measurements. The optical map of figure 22 shows the formation of a negative glow at $14.5 \mathrm{~cm}$ and of a $1 \mathrm{~cm}$-long Faraday dark space which shrinks in time to less than $1 \mathrm{~mm}$ as the lamp current increases. This evolution is also associated with a gradual displacement of the potential gradient toward the ground electrode in the space between 5 and $14.5 \mathrm{~cm}$. Some plasma striations, whose likely origin is a Frank and Hertz effect, are also observed in the cathode side of the homogeneous discharge channel (nascent positive column).

In ignitions at positive potential the electron flux in the lamp flows toward the active electrode since it is driven as an anode. It results that the pre-breakdown wave is fed by electrons that are generated at the wave front or ahead of it in the neutral gas rather than from the active electrode, as it is the case at negative applied potentials. The positive polarity of the pre-breakdown wave front causes an electric field which is directed away from it and ensures the collection of electrons from the neutral surroundings. In our experiments it is observed that the spread in the measured positions of the wave is less than $10 \mathrm{~ns}$. This very narrow statistical spread ensures the repeatability of the measurements but also indicates that the statistical time of first electron appearance is also less than $10 \mathrm{~ns}$, which is shorter than the formative lag time (i.e. propagation time) of the ionization wave by three orders of magnitude. In this situation it can be considered that the primary electrons feeding the ionization wave appear instantaneously [13], meaning that the source of primary electrons is not radioactivity and fast particles from the cosmic shower. This source has to be controlled by the ionization wave itself and there are four possible electron emission mechanisms that could be involved in this process:

1/ photo-ionization of the gas ahead of the wave front

$2 /$ secondary emission from the wall by ion impact

$3 /$ secondary emission from the wall by impact with excited and metastable atoms

4/ photoelectron emission (or electron detachment) from the wall due to UV radiation

The first possibility is ruled out because the low pressure leads to a too low atomic density for this process to be effective [45]. Moreover, there is no molecular species (impurities) or penning mixture which could lead to indirect photoionization. In the second case of secondary emission by ion impact to the wall, we assess the possibility of this mechanism by calculating the transit time of argon ions from the lamp axis to the wall. The E field amplitude in the wave front averaged in the 5-8 $\mu$ s period is $175 \mathrm{~V} / \mathrm{cm}$, corresponding to an ion mobility of $100 \mathrm{~cm}^{2} \mathrm{~V}^{-1} \mathrm{~s}^{-1}$ [46] which leads to an ion drift velocity of $175 \mathrm{~m} / \mathrm{s}$. The transit time over one radius is thus $2.85 \mathrm{~ms}$, which rules out this second mechanism. In the third mechanism of secondary emission via impact with atoms in the excited states we also estimate the transit time of argon atoms across one radius length from the mean thermal speed of argon atoms. At $300 \mathrm{~K}$ this mean velocity is $400 \mathrm{~m} / \mathrm{s}$, giving a transit time of $125 \mu \mathrm{s}$ or one order of magnitude higher than the wave propagation time, effectively ruling out this mechanism as well. The fourth mechanism of photoelectron emission from the dielectric wall [47] is therefore the most likely source for the primary electrons that feed the wave. Since the energy gap between the valence and 
conduction band of dielectrics like the glass tube used here exceed $5 \mathrm{eV}$, it is expected that the radiation responsible for the photoelectric emission arises from the resonant transitions of argon occurring from $79.77 \mathrm{~nm}(15.5 \mathrm{eV})$ to $106.67 \mathrm{~nm}(11.6 \mathrm{eV})$. The resonant nature of this radiation leads to radiation trapping in the argon gas with the consequence that UV photons reach the wall by diffusion via successive absorptions and re-emissions. This trapping process must therefore lead to a very local source of photoelectrons located in the immediate vicinity of the radiation source; this explains the confined nature of the ionization wave front of positive polarity as observed in the experiments.

\subsection{Wave propagation characteristics}

The recorded positions of the pre-breakdown wave via optical (CCD) and electrostatic (RCP) diagnostics (figure 24) are consistent with each others since they present similar trends. However, the absolute values present an offset similar to that observed in section 3.2, where the peak of $E$ field is located ahead of that of light emission. As in the case of negative ignitions this offset is longer at lower applied voltages, it is $1 \mathrm{~cm}$ at $+450 \mathrm{~V}$ and decreases to $0.4 \mathrm{~cm}$ at $+600 \mathrm{~V}$ and then becomes imperceptible at $+700 \mathrm{~V}$. This offset finds the same origins as those outlined earlier; the electron density in the region of peak $\mathrm{E}$ field strength is not high enough to lead to a maximum of argon excitation.

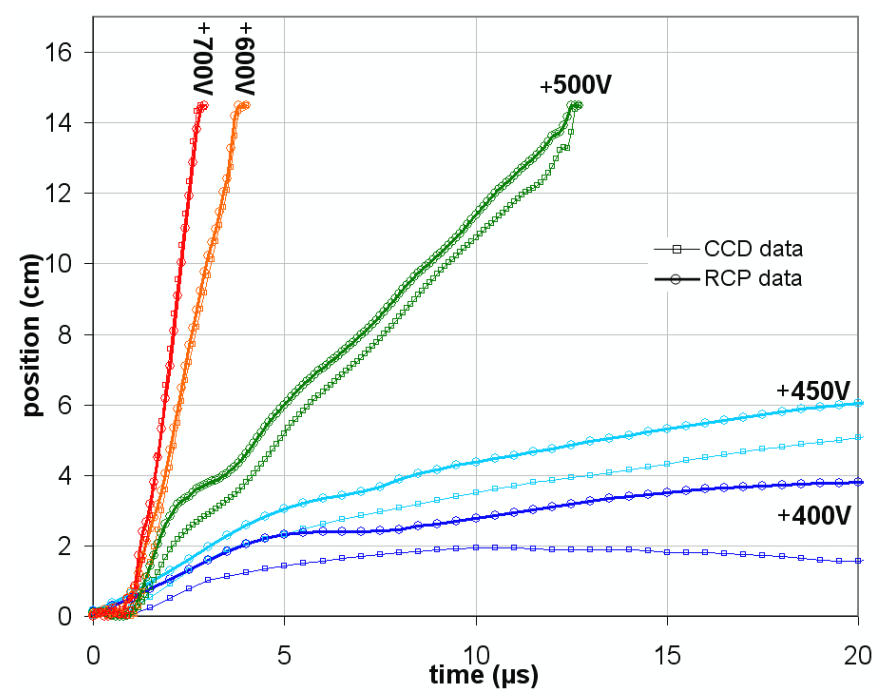

Figure 24: Measured position of the optical and of the E field peak of the pre-breakdown wave front in 3 Torr argon as a function of time and of the applied voltage amplitude. Ignitions below $+500 \mathrm{~V}$ are unsuccessful. The active and the ground electrodes are located at 0 and $14.5 \mathrm{~cm}$, respectively.

The propagation of the positive pre-breakdown wave is more linear in time than at negative potentials and also presents a transit time which decreases at higher applied voltages. However, it takes more time for the wave to complete its propagation at positive polarity for amplitudes below $+700 \mathrm{~V}$ and it is found that the wave does not reach the ground electrode at a $V_{A}$ value below $+500 \mathrm{~V}$, leading to an ignition failure. The condition for a successful lamp ignition stated in section 3.2 is thus also valid in the present case; the pre-breakdown has to reach the opposite electrode for the other processes to develop. 


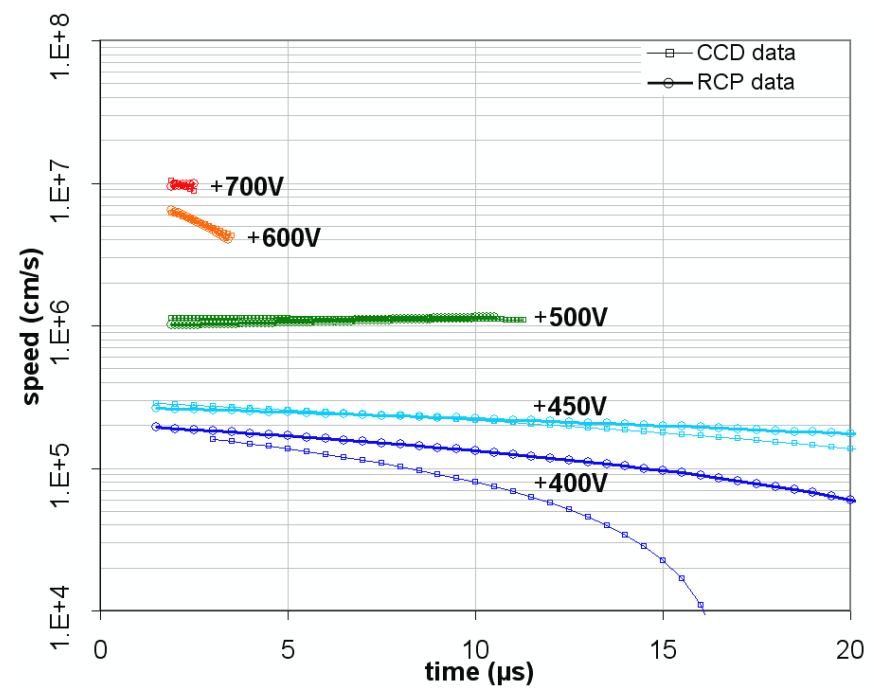

Figure 25: Calculated velocity of the optical and of the E field peak of the pre-breakdown wave front in 3 Torr argon as a function of time and of the applied voltage amplitude. Ignitions below $+500 \mathrm{~V}$ are unsuccessful.

The pre-breakdown velocities displayed in figure 25 are calculated from second order polynomials fitted to the position data. The evolution of the wave velocities is more constant in time than at a negative polarity but they present a trend which does not seem to be correlated to the applied voltage amplitude. At $+500 \mathrm{~V}$ the wave speed rises by a small factor in the course of its propagation while at higher voltages the trend is inversed and decreases quicker at $+600 \mathrm{~V}$ than at $+700 \mathrm{~V}$. Moreover, the wave velocities below $+600 \mathrm{~V}$ are lower than at negative polarity, but the average speed is still linearly dependant on $V_{A}$ although it presents a higher proportionality constant at positive potentials. Below the $+500 \mathrm{~V}$ threshold the wave speed continuously decreases in time toward zero as the ionisation wave extinguishes in the lamp. The same observation was made for negative polarity (section 3.2) but the evolution of the velocities associated with the peak of $\mathrm{E}$ field and of light emission is different from those shown in figure 13. At positive potentials the failed ignition waves have a light front which is slower than the E field peak, except at $+450 \mathrm{~V}$ before $7 \mu$ s. This difference could be explained by the fact that the failed pre-breakdown wave at positive polarity wanes out faster in time than at a negative polarity.

The measured return stroke positions from CCD and RCP data (figure 26) show a more linear propagation of this wave than at negative polarity. Furthermore, there is no clear separation between the peak of light emission and that of $\mathrm{E}$ field, except in the very early phases at $+500 \mathrm{~V}$. This indicates that the variation of electron density in the wave front region has a very flat slope (in fact flatter than at negative polarity), leading to the maximum of argon excitation coinciding with the peak of $E$ field and of reaction rate coefficient $K_{e x}$. This low spatial evolution of the electron density is an indication that there is no significant ionisation processes (and electron avalanche growth) that occur in the wave front. This analysis is consistent with the low E field strength of the wave front observed in section 4.1, and this leads to the conclusion that a positive-polarity return stroke is not an ionisation wave. 


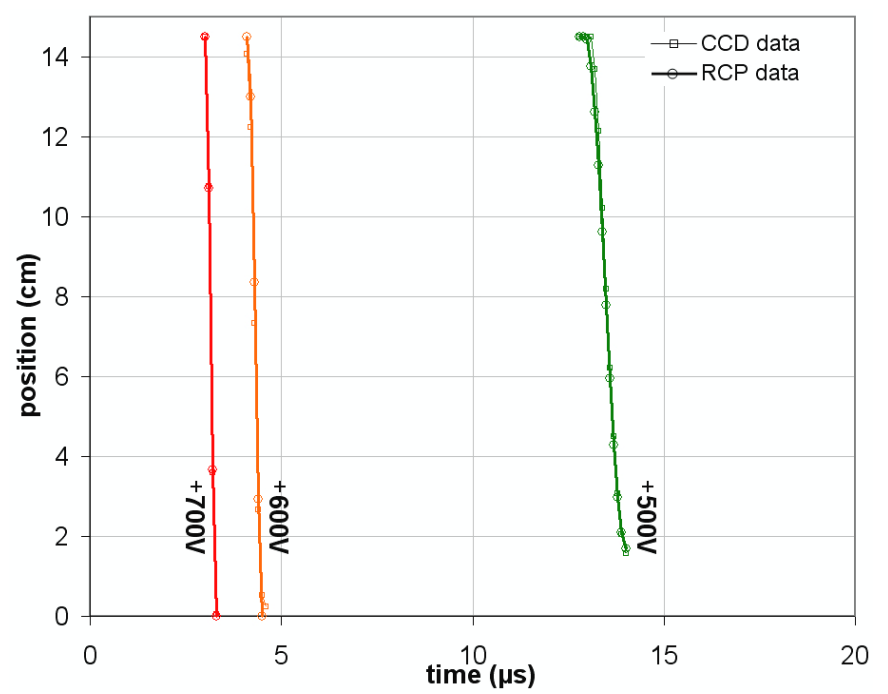

Figure 26: Measured position of the optical and of the E field peak of the return stroke wave front in 3 Torr argon as a function of time and of the applied voltage amplitude. The active and the ground electrodes are located at 0 and $14.5 \mathrm{~cm}$, respectively.

The steep slope of the evolution of the return stroke position as a function of time logically leads to very high wave velocities which are at least three times as high as those of pre-breakdown waves. The speed reaches its maximum near the middle of the propagation of the return stroke, which is clearly seen at $+500 \mathrm{~V}$ in figure 27 , and decreases by as much as one order of magnitude as the wave reaches the active electrode.

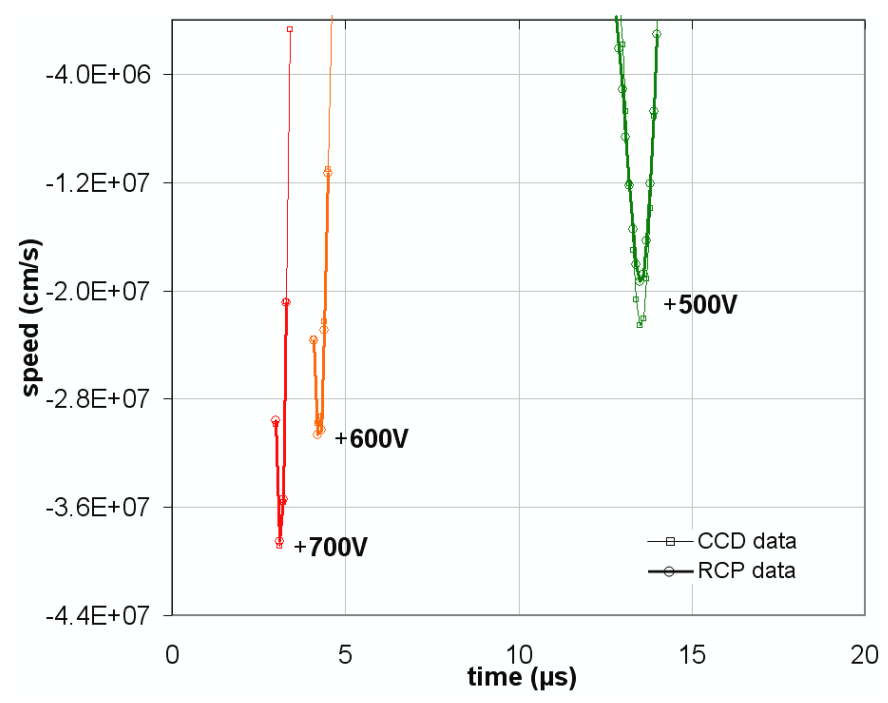

Figure 27: Calculated velocity of the optical and of the E field peak of the return stroke wave front in 3 Torr argon as a function of time and of the applied voltage amplitude.

\subsection{Electric field and potential evolutions}

The evolution in time of the wave front E field (figure 28) and potential (figure 29) in the case of ignitions at positive polarity differs markedly from the situation studied in section 3.3. One major 
difference is the lack of a direct relation between the E field evolution and the active electrode voltage, as other parameters may play more important roles during ignitions at positive polarity. During the 2 $\mu$ s voltage rise time, the pre-breakdown wave front potential and E field amplitude steadily increase with a slope which is proportional to the applied voltage $\left(60.1 \mathrm{MVs}^{-1}\right.$ and $27.7 \mathrm{MVcm}^{-1} \mathrm{~s}^{-1}$ at $+400 \mathrm{~V}$ versus $217.8 \mathrm{MVs}^{-1}$ and $113.7 \mathrm{MVcm}^{-1} \mathrm{~s}^{-1}$ at $+700 \mathrm{~V}$ ).

The first waves associated with a successful ignition $\left(V_{A}\right.$ higher than $\left.+450 \mathrm{~V}\right)$ reach a peak of potential and of E field at between 2.0 and $2.6 \mu \mathrm{s}$, at the exception of $+500 \mathrm{~V}$ which reaches its maximum at $3.4 \mu \mathrm{s}$. After this peak the E field amplitude remains relatively constant at a level which is the highest at $+500 \mathrm{~V}(178 \mathrm{~V} / \mathrm{cm})$, decreases to a minimum at $+600 \mathrm{~V}(137 \mathrm{~V} / \mathrm{cm})$ and then re-increase at $+700 \mathrm{~V}(160 \mathrm{~V} / \mathrm{cm})$. One explanation for this characteristic is that the width of the wave front increases with the applied voltage. The maximum E field level observed at $+500 \mathrm{~V}$ arises from a short wave front length, while the field re-increase at $+700 \mathrm{~V}$ finds its origin from a higher wave front potential. The plateau in the $\mathrm{E}$ field strength is not observed for negative ignitions due to differences in the voltage drop across the wake and in the evolution of the wave front length. As opposed to negative pre-breakdown waves, positive waves present a constant wave front profile during their propagation and a lower potential drop across the wake, both resulting in a more constant wave front $\mathrm{E}$ field strength over time.

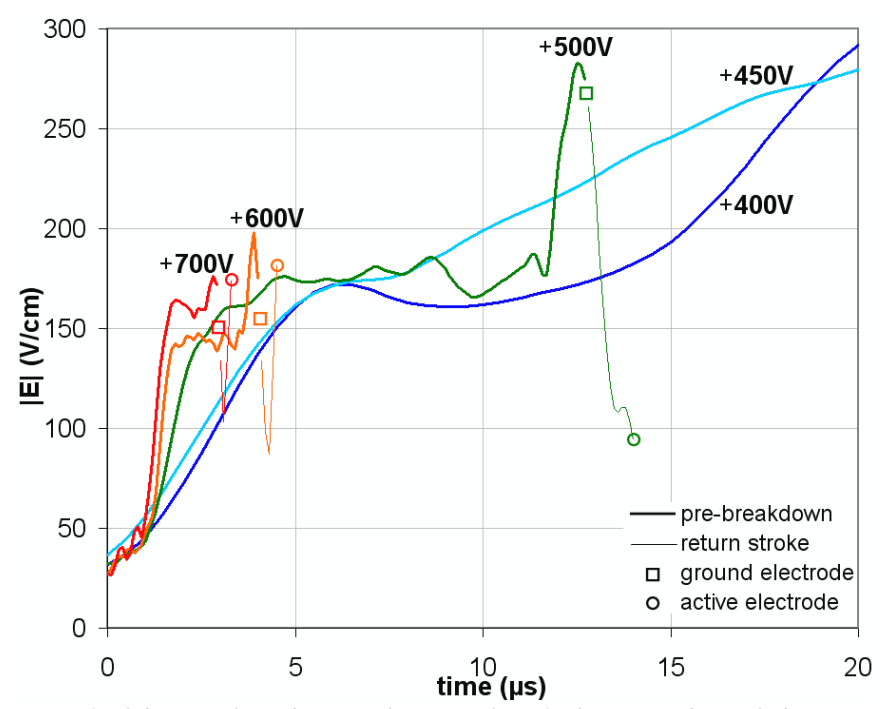

Figure 28: Electric field amplitude at the peak of the pre-breakdown wave and of the return stroke in 3 Torr argon as a function of time and of the applied voltage amplitude. Ignitions below $+500 \mathrm{~V}$ are unsuccessful.

The evolutions of the field strength and of the potential associated with failed ignitions below $+500 \mathrm{~V}$ present a peak at between 5 and $6 \mu \mathrm{s}$ and then the E field steadily increases in time to reach $308 \mathrm{~V} / \mathrm{cm}$ at $22.5 \mu \mathrm{s}$ at $+400 \mathrm{~V}$, and $557 \mathrm{~V} / \mathrm{cm}$ at $55 \mu \mathrm{s}$ for $+450 \mathrm{~V}$. These field values are significantly higher than those associated with pre-breakdown waves of successful ignitions, and the fact that the wave still wanes out in the lamp has to do with the extinction of the primary electron current source at or ahead of the wave front. This extinction is most likely caused by an insufficient emission of UV photons from the wave front which does not enable enough charges to be created to compensate for charge losses to the wall. During the same period the potential of the failed breakdown wave front decreases to $105 \mathrm{~V}\left(V_{A}=+400 \mathrm{~V}\right)$ or slightly increases to $247 \mathrm{~V}(+450 \mathrm{~V})$, leading to the conclusion that this observed rise in the field strength finds its origin in the decrease of the length of the wave front region 
during the wave extinction. At equilibrium the width of the static potential gradient is calculated to be $3.4 \mathrm{~mm}$ at $+400 \mathrm{~V}$ and $4.4 \mathrm{~mm}$ at $+450 \mathrm{~V}$.

When the pre-breakdown wave reaches the ground electrode its wave front potential sharply decreases while the local $\mathrm{E}$ field strength increases to peak values which are higher at lower $V_{A}$ levels $(176.0 \mathrm{~V} / \mathrm{cm}$ at $+700 \mathrm{~V}$ versus $283 \mathrm{~V} / \mathrm{cm}$ at $+500 \mathrm{~V})$. The peak of this E field increase coincides with the dip of the voltage decrease; the high observed $\mathrm{E}$ field values therefore result from a closing distance between the ionisation wave and the ground electrode, which may be smaller at the end of wave propagation for lower applied voltages. The peak E field strength exceeds those from negative pre-breakdown waves by a factor of up to 3. This enhanced local E field causes some ionisation to occur in the space between the pre-breakdown wave and the ground electrode and sets the formation of the return stroke.

As opposed to ignitions at negative polarity, the present return waves are associated with an E field strength which first decreases in the vicinity of $100 \mathrm{~V} / \mathrm{cm}$ and then increases back toward its initial amplitude (at the exception of the case at $+500 \mathrm{~V}$ ). The potential of the wave front of these return strokes increases nearly linearly during propagation to values equal or slightly lower than the active electrode potential, the difference never exceeding $50 \mathrm{~V}$. The decrease in the return stroke wave front E field amplitude despite its potential increase is most likely caused by a potential gradient which spreads during the wave propagation and reaches a maximum extension when the wave front is located mid way between the electrodes. At this point the estimated wave front length ranges from 3.3 to 4.0 $\mathrm{cm}$. As the return stroke propagates further, either a shrinking of the wave front or its steady potential increase (or both) leads to the observed rise of the peak E field strength.

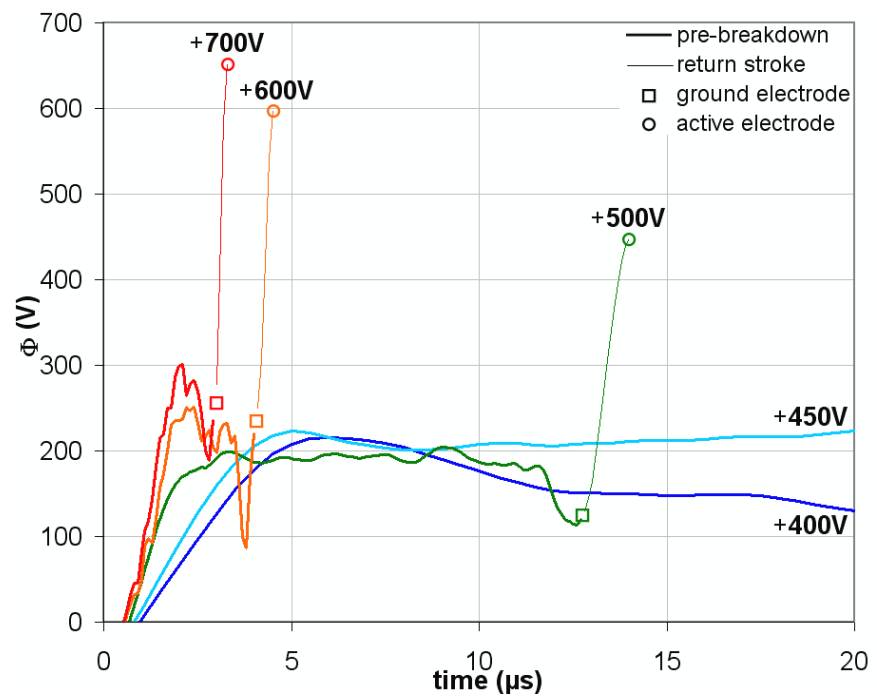

Figure 29: Electrostatic potential at the peak of the pre-breakdown wave and of the return stroke in 3 Torr argon as a function of time and of the applied voltage amplitude. Ignitions below $+500 \mathrm{~V}$ are unsuccessful.

\subsection{Current and net charge evolutions}

The evolutions in time of the displacement current (figure 30) and of the total net lamp charge (figure 31) are comparable with the observations made in section 3.4. One exception concerns the polarity of the current and of the lamp charge that are inverted. The measured total lamp displacement current during the pre-breakdown phase mostly originates from the ionisation wave front since there is nearly no potential evolution in its wake (figure 22), except for failed ignitions (section 4.5). 


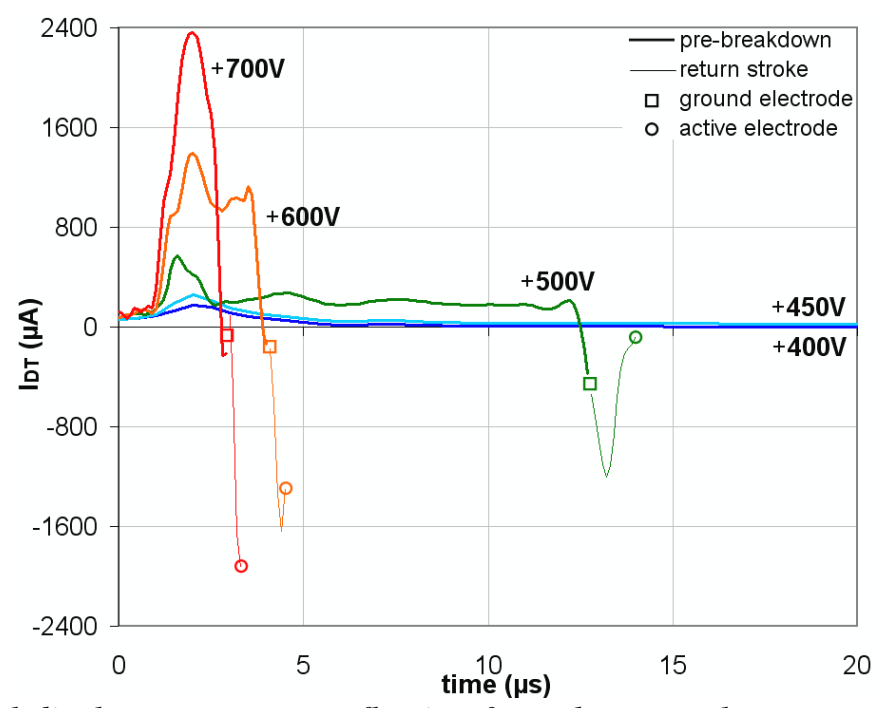

Figure 30: Total displacement current flowing from the argon lamp as a function of time and of the applied voltage amplitude. The current is positive from the lamp to the Faraday cage. Ignitions below $+500 \mathrm{~V}$ are unsuccessful.

The pre-breakdown waves are associated with a positive displacement current which charges the lamp to a positive potential. The current presents a peak at $2 \mu$ s which coincides with the end of the voltage rise time of the active electrode, and has an amplitude nearly proportional to $\mathrm{V}_{\mathrm{A}}$. The peak values range from $550 \mu \mathrm{A}$ at $+500 \mathrm{~V}$ to $2365 \mu \mathrm{A}$ at $+700 \mathrm{~V}$, and therefore exceed those of negative polarity ignitions by a factor of two. After $2.6 \mu$ s the total displacement current remains relatively constant $(220 \mu \mathrm{A}$ at $+500 \mathrm{~V}$ and $1060 \mu \mathrm{A}$ at $+600 \mathrm{~V})$ as opposed to the steady decrease observed at negative polarity. This plateau occurs at $0.6 \mu$ s after the end of the voltage rise time period due to influences from the anode sheath which forms in front of the active electrode in this time period (section 4.1). The plateau is not observed at $+700 \mathrm{~V}$ since the corresponding pre-breakdown wave is half way through the inter-electrode distance at $2 \mu \mathrm{s}$ and has nearly finished its propagation at $2.6 \mu \mathrm{s}$, at the end of the influence of the nascent anode sheath.

Once the pre-breakdown wave completes its propagation to the ground electrode the lamp reaches its maximum electrostatic charge and the displacement current reverses its polarity. The return stroke which starts as a result of the local E field enhancement at the ground electrode leads to the reversal of the total displacement current (figure 30) which discharges the lamp and results in a potential redistribution in the inter-electrode space (figure 22). For a given voltage amplitude, the lamp charging is higher at positive polarity.

The maximum lamp net charge $Q_{T m}$ reached at the end of the pre-breakdown wave propagation does not correspond to a lamp fully charged up to the active electrode potential. The data of the equivalent length $L_{E}$ and capacitance $C_{E}$ in table 2 show a charging capacity which reaches up to $90 \%$, higher for successful ignitions than at negative polarity (table 1). This high charging capacity is a direct consequence of the low potential gradient of the wave of the pre-breakdown wave, which turns out to be charged at a nearly constant potential close to that of the active electrode. The equivalent length also has a higher value at +500 and $+600 \mathrm{~V}$ than at negative potentials, meaning that the wave propagates farther away from the active electrode than at negative potentials in order to enable the bridging of the gap between the wave front of the ground electrode. 


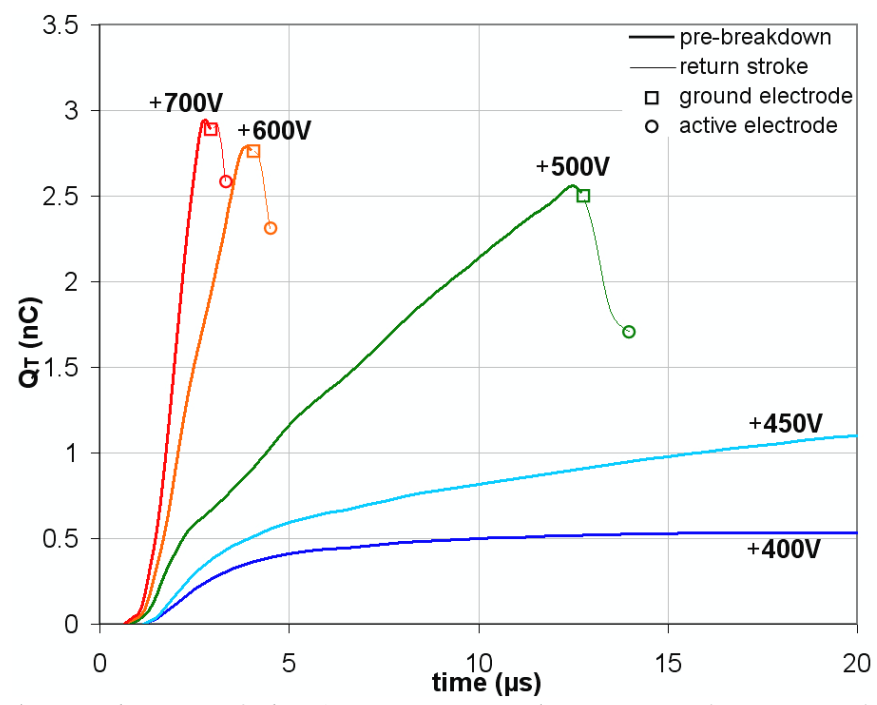

Figure 31: Total net charge of the 3 Torr argon lamp as a function of time and of the applied voltage amplitude. Ignitions below $+500 \mathrm{~V}$ are unsuccessful.

This characteristic could be explained by the confined nature of the positive wave front where the primary electrons are generated by photoemission from the wall, in the close vicinity of the charge multiplication region. Since the ground electrode is at room temperature, primary electrons from there have to be emitted via the photoelectric effect from its oxide emitter whose work function is typically $2.5 \mathrm{eV}$ [48]. The maximum photon wavelength that could lead to photoemission is $496 \mathrm{~nm}$, meaning that non-resonant argon emission (such as the $3 p^{5} 5 p \rightarrow 3 p^{5} 4$ s cluster) from the wave front can lead to electron emission from the ground electrode. Since this radiation is not absorbed by the argon gas, the influence of the ground electrode irradiation can occur over a relatively long range. At $+700 \mathrm{~V}$ the gap is bridged at a longer range $(6.7 \mathrm{~cm})$ than at negative potential $(4.2 \mathrm{~cm})$. This change of situation arises most likely as a result of the higher wave front potential which leads to a higher E field strength in the gap. A calculation of the average E field strength in the gap between the wave front and the ground electrode at the moment of bridging (section 3.4) reveals an amplitude of 35.2 and $39.3 \mathrm{~V} / \mathrm{cm}$ at +700 and $+600 \mathrm{~V}$, respectively. These figures are remarkably consistent with those calculated for ignitions at negative polarity, which might indicate a fundamental origin in the average $\mathrm{E}$ field required for the bridging of the wave front - ground electrode gap. There is however one departure at $+500 \mathrm{~V}$ which yields an average $\mathrm{E}$ field in the gap of $126.4 \mathrm{~V} / \mathrm{cm}$. This higher magnitude could be caused by a much lower density of photoelectrons generated from the ground electrode due to less light being emitted from the wave front. This would thus require a higher field strength in order to produce a certain minimum amount of free electrons to close the gap.

Table 2: Measured total lamp net charge $Q_{T m}$ at the end of the pre-breakdown wave propagation and calculated equivalent charged capacitance $C_{E}$ and length $L_{E}$ as a function of the applied voltage level $V_{A}$.

\begin{tabular}{|c|c|c|c|}
\hline $\boldsymbol{V}_{\boldsymbol{A}}(\mathbf{V})$ & $\boldsymbol{Q}_{\boldsymbol{T}}(\mathbf{n C})$ & $\boldsymbol{C}_{\boldsymbol{E}}(\mathbf{p F})\left[\% \boldsymbol{C}_{\boldsymbol{T}}\right]$ & $\boldsymbol{L}_{\boldsymbol{E}}(\mathbf{m m})$ \\
\hline+400 & 0.535 & $1.338[23.6]$ & 35.4 \\
+450 & 1.369 & $3.042[53.7]$ & 80.5 \\
\hline+500 & 2.560 & $5.120[90.3]$ & 135.5 \\
\hline+600 & 2.787 & $4.645[81.9]$ & 122.9 \\
\hline+700 & 2.945 & $4.207[74.2]$ & 77.9 \\
\hline
\end{tabular}


Like in section 3.4 it is found that the equivalent length and capacitance follow a linear relationship with $V_{A}$ in the case of successful ignitions, which for $C_{E}$ yields:

$$
C_{E}=C_{T}\left(-8.05 \cdot 10^{-4} \cdot V_{A}+1.30\right)
$$

Relation (16) presents a slope which is three times as steep as that of equation (15), leading to a relative lamp charging capacity which is more dependent on the lamp voltage than at negative polarity. Pre-breakdown waves of positive polarity require more charges for their propagation at a given voltage level, and a higher electrical conductivity in their wake leads to a wave front potential which remains closer to that of the active electrode. It results that below $+700 \mathrm{~V}$ the equivalent length $L_{E}$ is higher than at negative polarity because the determining parameter in the wave propagation is the amount of charges that needs to be supplied to the lamp. As the applied voltage level increases toward $+700 \mathrm{~V}$, the effect of the higher wave front potential becomes predominant, and this leads to an $L_{E}$ value which becomes lower than at negative polarity.

Interestingly the lamp charging capacity for failed ignitions at positive potentials (table 2) are markedly lower $\left(53.7 \%\right.$ of $C_{T}$ at $\left.+450 \mathrm{~V}\right)$ than at negative potentials $\left(65.3 \%\right.$ of $C_{T}$ at $\left.-300 \mathrm{~V}\right)$. The equivalent length is also shorter by $1.8 \mathrm{~cm}$, which indicates that the positive pre-breakdown wave loses its ability to properly propagate much faster below the ignition voltage. This could be related to a minimum level of UV radiation that needs to be generated by the wave front in order to sustain its propagation via the generation of its photoelectron current.

The propagation of the return stroke is associated with a decrease of the net lamp charge in the same way as observed for ignitions at negative polarity. However, the level of lamp charge at the end of the return stroke propagation corresponds to an average lamp potential which is much higher than half the applied voltage. This departure finds its origin in a larger voltage drop $\Delta V_{K+A}$ at the electrodes due to the sheaths. This voltage drop can be estimated from the total net charge $Q_{T f}$ at the end of the return stroke propagation if we consider a constant voltage gradient in the length of the plasma channel that bridges the electrodes, and a negligible thickness of the electrode sheaths:

$$
\Delta V_{K+A}=2 \cdot \frac{Q_{T f}}{C_{T}}-V_{A}
$$

From the experimental data we find a total electrode voltage fall of $110 \mathrm{~V}$ for an applied $+500 \mathrm{~V}$ and this value rises by a factor of nearly 2 at higher applied voltages $(214 \mathrm{~V}$ at $+600 \mathrm{~V}$ and $210 \mathrm{~V}$ at $+700 \mathrm{~V}$ ). These values contrast with those related to ignitions at negative polarity, which yield a total electrode voltage drop comprised between 14 and $40 \mathrm{~V}$ (calculated from data presented in section 3.4). This difference arises as the cathode in the negatively-driven lamp is the active electrode heated to $1000 \mathrm{~K}$ which therefore feeds the discharge via thermionic electron emission. In the positively-driven lamp the cathode is the ground electrode, and its low temperature leads to the formation of a high voltage sheath needed for the emission of electrons via secondary processes. This sheath leads therefore to a higher average lamp potential after the propagation of the return stroke.

\subsection{Failed positive ignition}

The optical recording of the pre-breakdown wave associated with a failed ignition at $+450 \mathrm{~V}$ (figure 32) shows a very narrow light emission profile associated with the ionisation processes, whose evolution is similar to that observed in section 4.1. The first process to occur upon the voltage application to the active electrode is the neutralization of the negative space charges left by the pre- 
ionisation pulse applied prior to the main positive voltage pulse. This neutralization leads to a burst of displacement current at $1 \mu \mathrm{s}$, and to the start of the pre-breakdown wave which progresses inside the lamp with a constant width but with a decreasing light intensity and a slope which becomes steeper over time. This wave finally extinguishes at $32 \mu$ s and no light emission is observed in the remaining $28 \mu$ s of the recording.

The potential map related to this ionisation wave shows a narrow potential gradient which is associated with the pre-breakdown wave front. Its width continuously decreases in time and space and the wave continues its propagation and narrowing after the extinction of its light emission up to $45 \mu \mathrm{s}$ when it becomes stationary. From this point the wave front develops a negative space charge at $10 \mathrm{~cm}$, whose origin is not fully understood. This ionisation wave is also associated with a wave of displacement current (figure 32) which follows the evolution of the light emission and also continues its propagation until $45 \mu \mathrm{s}$ but remains non zero in strength until the end of the recording. This suggests that ionisation processes and charge redistribution still occur after the observed end of optical emission. The total lamp net charge reaches a maximum value of $1.37 \mathrm{nC}$ at $41.5 \mu \mathrm{s}$ and then decreases to $1.22 \mathrm{nC}$ at $59.5 \mu \mathrm{s}$. This evolution is most likely cause by the collection of free electrons at the active electrode. A new feature is present which was not observed at higher applied voltages: a potential gradient in the wake of the pre-breakdown wave which certainly results from a low electrical conductivity of this ionised medium.
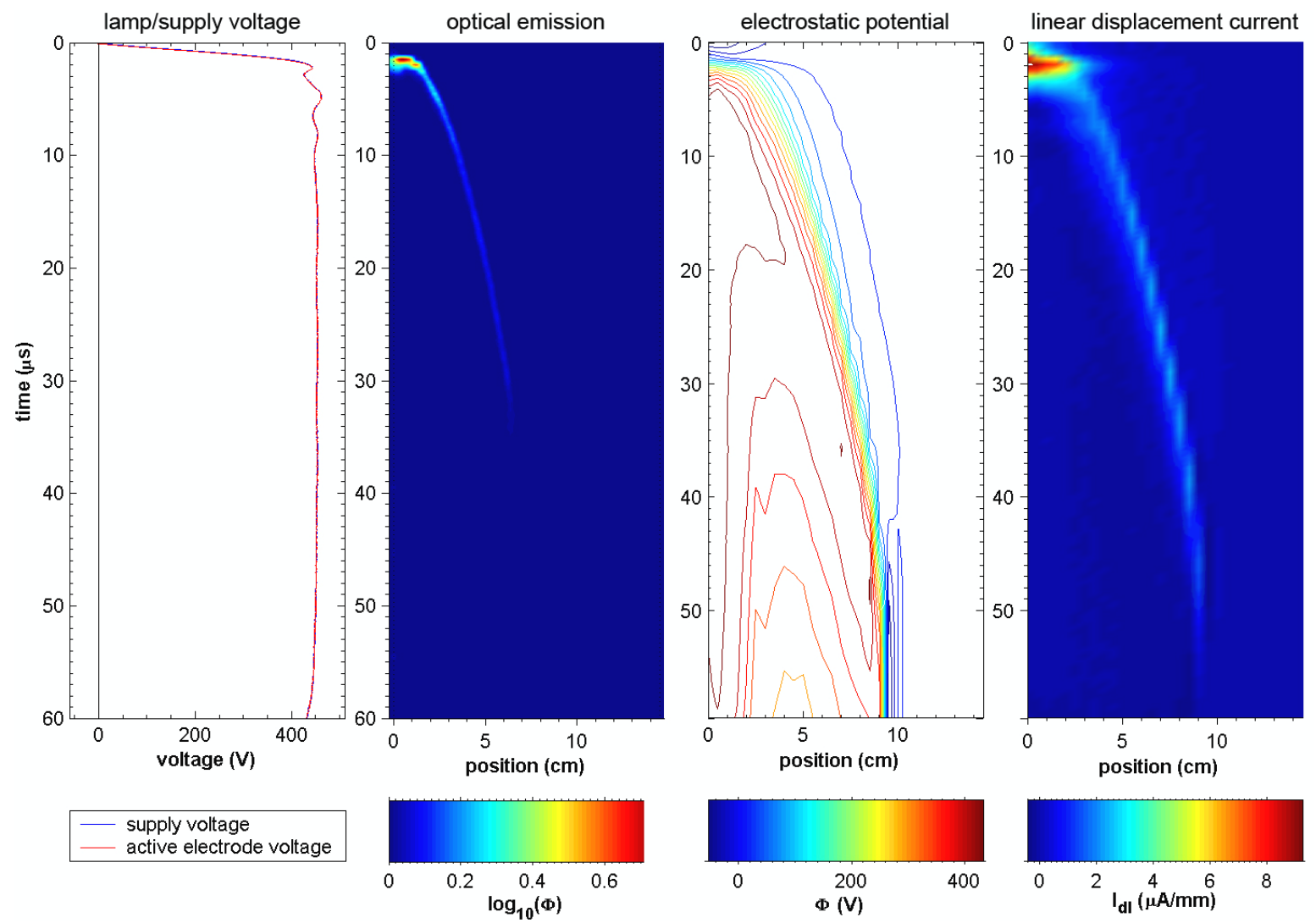

Figure 32: Space-time diagrams of the evolution of the measured light emission, of the net electrostatic potential and of the calculated linear displacement current (positive from the lamp to the Faraday cage) associated with failed breakdown phenomena occurring in 3 Torr argon at $+450 \mathrm{~V}$. The active and the ground electrodes are located at 0 and 14.5 cm, respectively. 
The evolution and narrowing of the potential gradient over time leads to the increased $\mathrm{E}$ field strength observed in figure 28 of section 4.3 and in the E field maps of figure 33. The E field amplitude reaches up to $557 \mathrm{~V} / \mathrm{cm}$ as a result of the narrowing of the potential gradient to $4.4 \mathrm{~mm}$. This profile narrowing results in the observed stepped profiles of the potential gradient, of the displacement current and of the electric field due to the electric potential feature of the wave front being smaller than the width of the $\mathrm{RC}$ probe $(5 \mathrm{~mm})$. The potential gradient in the wake of the ionisation wave results in a $\mathrm{E}$ field strength in the order of $100 \mathrm{~V} / \mathrm{cm}$.

The field angle map of figure 33 reveals that the E field vector at the wave front remains at about $+100^{\circ}$ during its propagation, which is similar to the case observed for successful ignitions (section 4.1). The negative space charge that develops in the front of the stalling wave at $42 \mu$ s leads to a local reversal of the E field vector which points at around $+135^{\circ}$ at $9.7 \mathrm{~cm}$ and shifts to $-70^{\circ}$ at $10.2 \mathrm{~cm}$. Starting from $20 \mu \mathrm{s}$ a second field reversal develops in the wake of the ionisation wave, leading after $35 \mu$ s to a field vector which points at $+90^{\circ}$ at $2.5 \mathrm{~cm}$ and reverses to $-30^{\circ}$ between 6 and $8 \mathrm{~cm}$. This reversal is most likely caused by the build-up of a negative space charge in the lamp between 2.5 and $8 \mathrm{~cm}$ from the active electrode.
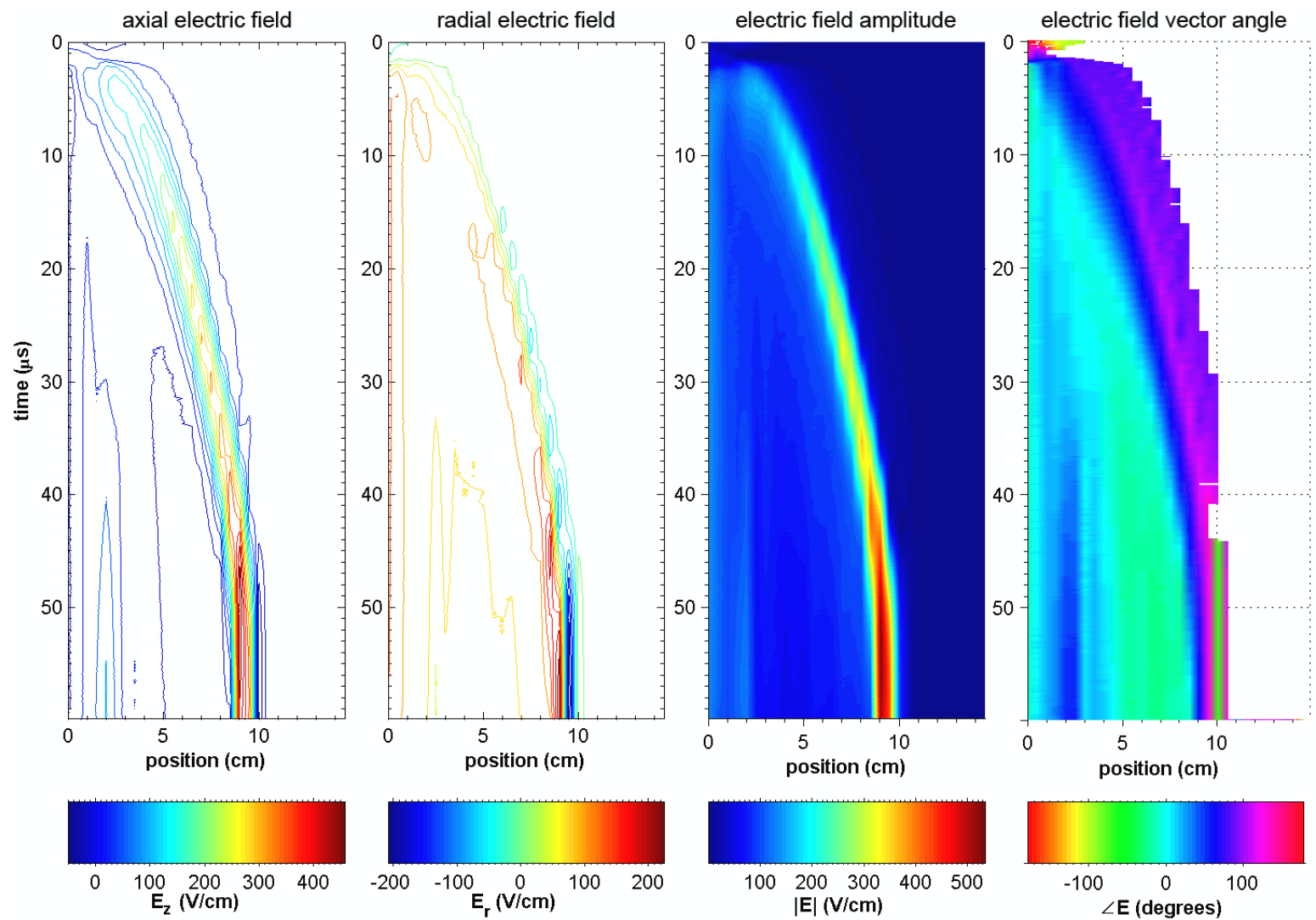

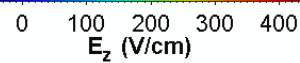

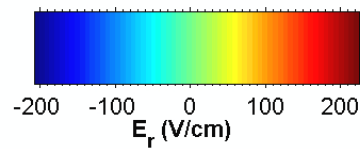

$|\mathrm{E}|(\mathrm{V} / \mathrm{cm})$

Figure 33: Space-time diagrams of the evolution of the axial electric field (positive from the active to the ground electrode), of the radial electric field (positive from the lamp to the Faraday cage), of the electric field amplitude and vector angle (null when oriented from the lamp wall to the Faraday cage, and oriented from the active to the ground electrode at $+90^{\circ}$ ) associated with failed breakdown phenomena in 3 Torr argon at $+450 \mathrm{~V}$. The active and the ground electrodes are located at 0 and $14.5 \mathrm{~cm}$, respectively. 


\subsection{Summary of positive ignition processes}

Successful ignitions at a positive polarity above $+450 \mathrm{~V}$ present the same three phases as described in previous section. There is a first propagation of a pre-breakdown wave from the active to the ground electrode, followed by a return stroke which propagates backward and draws a homogeneous plasma channel in its wake. The final stage is an increase in intensity of this channel until the lamp reaches a steady-state operation. However, the nature and propagation of the two waves differ markedly from those at negative polarity and the associated current flows are reversed.

The pre-breakdown wave at positive polarity presents a very narrow light emission profile associated with a narrow potential gradient which leads to a strong and localized electric field whose vector angle remains essentially axial during the wave propagation. The pre-breakdown wave is associated with a displacement current which flows from the lamp to the Faraday cage, resulting in the positive electrostatic charging of the lamp which reaches a maximum at the end of the wave propagation. The wave front velocity, electric field strength and intensities of the emitted light and of the displacement current all remain nearly constant during the wave propagation, and are caused by a relatively constant wave front potential and the peculiar nature of the source of primary electrons feeding the wave front. The polarity of this ionization wave leads to an electron current which flows from the wave front to the active electrode. It then results that the source of primary electrons that feeds the wave originates from the wave front, or ahead of it. It is found that the most likely source is photoelectric emission from the dielectric wall upon irradiation with UV photons that are created from the electron avalanche in the wave front.

The motion of the pre-breakdown wave toward the ground electrode also leads to an increase of the local electric field in front of this electrode. It is found that the bridging between the wave and the ground electrode occurs at the same average E field strength as for negative potentials, except at $+500 \mathrm{~V}$ which requires a higher field amplitude $(126.4 \mathrm{~V} / \mathrm{cm})$ presumably because of a too low density of primary electrons. This bridging results in the closing of the electric circuit, the formation of the cathode sheath and the initiation of the return stroke. This wave propagates at much higher velocities than the pre-breakdown wave, and has an associated local electric field whose amplitude remains at the level or is below that of the first wave. Its effect is also a partial discharging of the lamp and a homogenization of the potential gradient between the electrodes.

The mechanisms of ignition failure which are observed below $+500 \mathrm{~V}$ are similar to those analysed at negative potentials; In this situation the pre-breakdown wave starts its propagation from the active electrode and extinguishes in the lamp before reaching the ground electrode. This also sets the condition for a proper lamp ignition not only in terms of minimum voltage $(+500 \mathrm{~V}$ in the present case), but also in terms of minimum voltage pulse duration $(12.5 \mu \mathrm{s})$ and of minimum amount of charges to be provided for the proper charging of the lamp $(2.56 \mathrm{nC}$ at $+500 \mathrm{~V})$. A failure to meet one of these criteria results in the partial propagation of the pre-ionisation wave and the partial positive charging of the lamp. It is observed that this charge remains in the lamp after the end of the wave propagation, leading to a memory effect which is likely to affect subsequent ignition attempts at positive potentials.

\section{Ionisation wave model and breakdown analysis}

A simple model is developed in this section in order to better understand the nature of pre-breakdown waves. The first part presents the outlines of a general model for the ionisation wave. Next, more details are given for the positive and negative waves. Finally the static ignition voltages are estimated from the analysis of the formative lag time of the pre-breakdown waves. 


\subsection{Modelling of the pre-breakdown wave}

A simple analytical model of the pre-breakdown wave is developed from the observation that its velocity depends on the rate of potential charging of the lamp [16]. A relation can therefore be drawn between the electric field $E_{F}$ in the ionisation wave front and its velocity. In the present model we consider the pre-breakdown wave front to have a linear potential decrease over a characteristic length $L_{F}$ as show in figure 34. The electric field strength in the wake of the wave is considered to have a much lower amplitude than in the front, resulting in the lack of ionisation processes in this region.

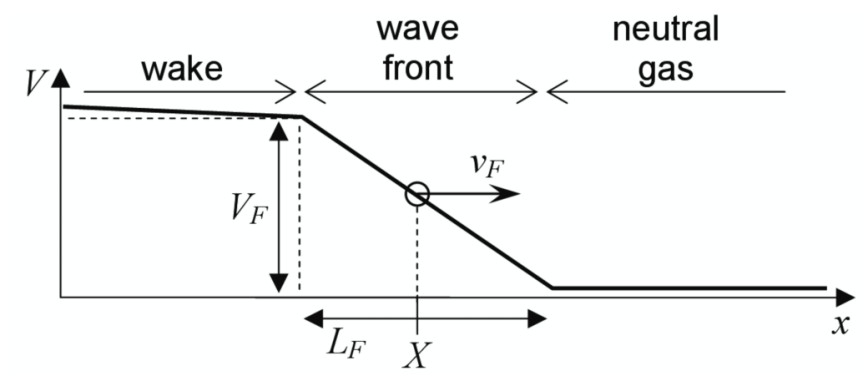

Figure 34: Model of the net lamp potential as a function of position in the region of the pre-breakdown wave front.

The rate of electric potential change over time at a given location $X$ along the lamp is then:

$$
\left.\frac{d V}{d t}\right|_{X}=\frac{v_{F} \cdot V_{F}}{L_{F}}=\left.v_{F} \cdot E_{F}\right|_{X}
$$

Where $V_{F}$ and $v_{F}$ are the potential at the ionisation wave boundary and the wave speed, respectively. The expression of the rate of potential charging of the lamp is derived from the equivalent circuit of figure 35 where $R_{F}$ and $R_{W}$ model the ohmic impedances of the ionisation wave front and of the wake of the pre-breakdown wave, respectively.

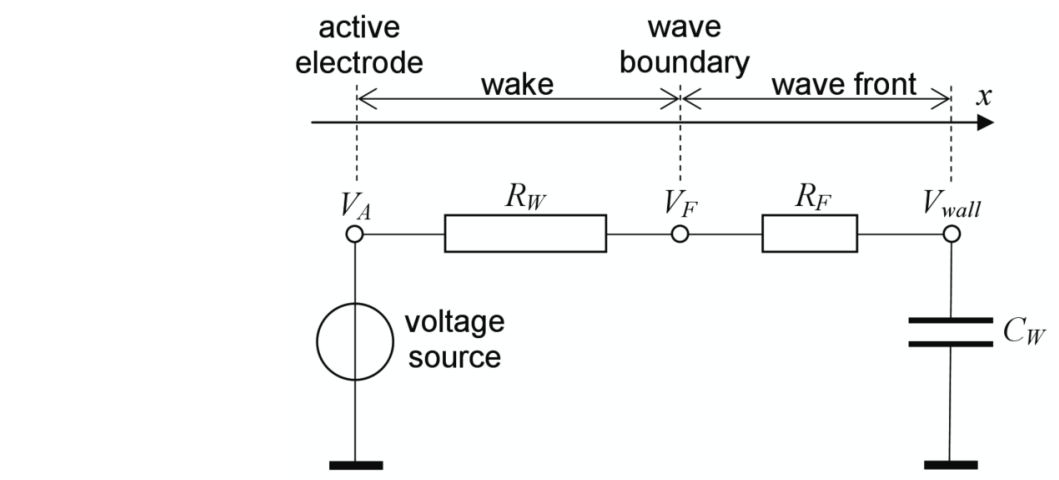

Figure 35: Static electrical and impedance model of a pre-breakdown wave.

In this model $C_{W}$ represents the total lamp capacitance to the ground in the length $L_{F}$ of the wave front. The rate of charging of the capacitor $C_{W}$, which defines the velocity of the wave, is thus expressed in terms of the potential at the wave boundary $V_{F}$ and of the ohmic impedance of the wave front such that: 


$$
\left.\frac{d V_{\text {wall }}}{d t}\right|_{t=0}=\frac{V_{F}}{R_{F} \cdot C_{W}}
$$

The rate of wall charging in equation (19) is the slope of the charging rate of $C_{W}$ expressed at $t=0$ because $V_{\text {wall }}$ in the equivalent circuit of figure 35 is always null since the wave front constantly moves toward a neutral and uncharged lamp section. The expression of the ionization wave velocity is found by combining equations (18) and (19), thus giving:

$$
v_{F}=\frac{V_{F}}{E_{F} \cdot R_{F} \cdot C_{W}}
$$

The impedance $R_{F}$ of the wave front represents a region in the lamp where Townsend-like ionisation occurs as a result of the local electric field, present there due to the gradual charging of the lamp (i.e. the axial potential gradient). The charge multiplication that results from the electron avalanche has the well known form derived from the Townsend theory of ignition:

$$
\Gamma_{e a}=\Gamma_{e 0} \cdot \exp \left(\alpha \cdot L_{F}\right)
$$

Where $\alpha$ is the first Townsend coefficient and $\Gamma_{e 0}$ the initial flux of electrons entering the wave front region. This expression is valid only in the case of free space, but in our situation the nascent plasma evolves inside a glass tube where charge loss by electron diffusion toward the wall occurs. This leads to a radial electron flux $\Gamma_{e l}$ which is not collected at the end of the wave front region, and which therefore does not contribute to the avalanche process (figure 36).

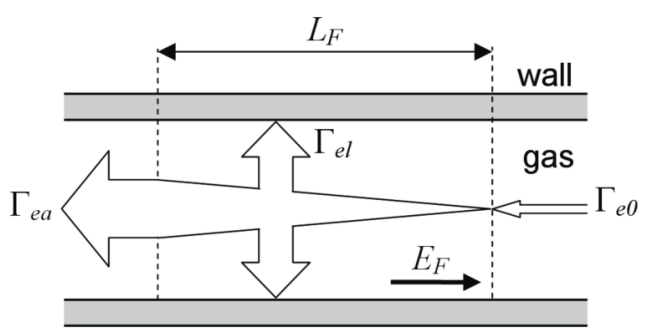

Figure 36: General model of the wave front region where there is charge multiplication by electron avalanche and charge loss to the wall via radial diffusion.

We integrate the expression of the loss rate of electrons to the wall by following the approach of Lisovskiy et al. [13]. In the wave front region we consider that the field $E_{F}$ is homogeneous and not affected by the charging wall. In this condition the rates of electron flux growth $\left.\left(d \Gamma_{e i} / d x\right)\right|_{x}$ and of loss $\left.\left(d \Gamma_{e l} / d x\right)\right|_{x}$ at a position $x$ in the region of interest are then:

$$
\begin{aligned}
& \left.\frac{d \Gamma_{e i}}{d x}\right|_{x}=\Gamma_{e}(x) \cdot \alpha \\
& \left.\frac{d \Gamma_{e l}}{d x}\right|_{x}=\Gamma_{e}(x) \cdot \beta
\end{aligned}
$$

Where the $\beta$ constant is related to the rate of electron loss via diffusion to the wall such as: 


$$
\beta=v_{l} / v_{d e}
$$

With $v_{d e}$ the electron drift velocity and $v_{l}$ the frequency of electron loss, equal to the inverse of the electron containment time (or diffusion time) $\tau_{l}$ in the ionized gas. Lisovskiy et al. point out that a quasi-neutral plasma is not yet formed before the discharge tube has completed its electrical breakdown (Townsend regime)[13]. As a consequence the ion flux to the wall is not coupled to that of electrons; the diffusion regime is free due to the low charge density and the resulting long Debye length which exceeds the discharge tube diameter. A normal Fickian diffusion in a cylindricallysymmetric ionized medium is therefore considered [49] and $\beta$ is thus expressed as:

$$
\beta=\frac{1}{\tau_{l} \cdot v_{d e}}=\frac{D_{e}}{v_{d e}}\left(\frac{R_{J 0}}{R_{L}}\right)^{2}
$$

where $D_{e}$ is the electron radial diffusion coefficient, $R_{L}$ the radius of the discharge tube and $R_{J o}$ the relative radius at the first zero point of the zeroth-order Bessel function $\left(R_{J_{0}}=2.405\right)$, a dimensionless parameter in the equation. The net rate of electron flux growth $d \Gamma_{\mathrm{e}} / d x$ at a position $x$ can be expressed from relations (22) and (23):

$$
\left.\frac{d \Gamma_{e}}{d x}\right|_{x}=\left.\frac{d \Gamma_{e i}}{d x}\right|_{x}-\left.\frac{d \Gamma_{e l}}{d x}\right|_{x}=\Gamma_{e}(x) \cdot(\alpha-\beta)
$$

The net electron flux $\Gamma_{e a}$ collected at the end of the electron avalanche is thus:

$$
\Gamma_{e a}=\Gamma_{e 0} \cdot \exp \left((\alpha-\beta) \cdot L_{F}\right)
$$

The expression of $R_{F}$ is derived from $E_{F}$ and from the electron flux density $\Gamma_{e a}$ available after the avalanche and charge loss in the wave front, leading to the following relation:

$$
R_{F}=\frac{L_{F} E_{F}}{\pi R_{L}^{2} \Gamma_{e a} q_{e}}
$$

$q_{e}$, in (28), is the absolute value of the electron charge. Next, $\Gamma_{e a}$ is expressed in terms of the field strength $E_{F}$ and of the electron mobility $\mu_{e}$ and initial density $n_{e 0}$ at the start of the electron avalanche:

$$
\Gamma_{e a}=n_{e 0} \mu_{e} E_{F} \exp \left((\alpha-\beta) \cdot L_{F}\right)
$$

Giving the following expression for $R_{F}$ :

$$
R_{F}=\frac{L_{F}}{\pi R_{L}^{2} n_{e 0} q_{e} \mu_{e} \exp \left((\alpha-\beta) \cdot L_{F}\right)}
$$

The expression of the wave front velocity as a function of $n_{e 0}$ is thus obtained by combining (20) with (30), giving:

$$
v_{F}=\frac{\pi R_{L}^{2} n_{e 0} q_{e} \mu_{e} \exp \left((\alpha-\beta) \cdot L_{F}\right)}{L_{F} \cdot C_{l}}
$$


In (31) $C_{l}$ is the lamp linear capacitance which replaces $C_{W} / L_{F}$ in the expression. A relatively simpler and more useful expression of $v_{F}$ is derived by reformulating (31) into a function of the priming electron current $i_{e 0}$ that feeds the electron avalanche.

$$
v_{F}=\frac{i_{e 0} \exp \left((\alpha-\beta) \cdot L_{F}\right)}{E_{F} L_{F} C_{l}}
$$

Interestingly expression (32) shows that the wave velocity is negatively affected by a higher electric field strength. This can be understood by the fact that for a given velocity a higher $E_{F}$ amplitude leads to a larger $d V / d t$ value which logically requires a higher charging current level. This current will normally increase for higher E field strength because the Townsend coefficient $\alpha$ is also a function of this field magnitude. The $E_{F} \cdot L_{F}$ factor in (32) is the wave boundary potential $V_{F}$ which is defined by the voltage divider formed by the impedances $R_{F}$ and $R_{W}$ such that:

$$
V_{F}=V_{A} \frac{R_{F}}{R_{W}+R_{F}}
$$

The ohmic impedance $R_{W}$ of the wake of the wave is calculated by assuming that only charge loss to the wall occurs during the transport of electrons from one end of the wake to the other. The resulting relation between the electron fluxes at the wake inlet $\left(\Gamma_{e w i}\right)$ and at its outlet $\left(\Gamma_{e w o}\right)$ is then:

$$
\Gamma_{e w o}=\Gamma_{e w i} \cdot \exp \left(-\beta_{W} \cdot L_{W}\right)
$$

Where $L_{W}$ is the length of the wake, and $\beta_{W}$ is a constant related to the rate of electron loss via diffusion to the wall in this region. $\beta_{W}$ is also defined by (24) and (25) with a diffusion coefficient $D_{e}$ set by the charged state of the lamp and its electrostatic potential in regard to that of the active electrode and of the Faraday cage. It is assumed that the diffusion regime of electrons in this region is still free (i.e. a Debye length exceeding the discharge tube diameter). A derivation of $R_{W}$ in terms of the electron flux at the wake outlet leads to an expression similar to (28):

$$
R_{W}=\frac{L_{W} E_{W}}{\pi R_{L}^{2} \Gamma_{e w o} q_{e}}
$$

Where $E_{W}$ is the electric field strength in the wake, which is assumed to be constant and too weak to cause any ionization. A re-expression of (35) in terms of initial electron density $n_{\text {ewi }}$ at the wake inlet yields the following relation:

$$
R_{W}=\frac{L_{W}}{\pi R_{L}^{2} n_{e w i} q_{e} \mu_{e} \exp \left(-\beta_{W} \cdot L_{W}\right)}
$$

Relation 36 shows that the wake impedance depends on its length, as expected, and therefore increases during the wave propagation. However, $R_{W}$ depends also on the initial electron density and on the rate of electron loss to the wall. The first parameter is defined by the total wave impedance (i.e. $R_{W}+R_{F}$ ) and the active electrode voltage, while $\beta_{W}$ is primarily set by the state of lamp charging after the passage of the wave front and the lamp polarity in respect to the Faraday cage. 


\subsection{Pre-breakdown wave of negative polarity}

The expression of the wave velocity (32) developed in the model can be related to the experimental results by assuming that the measured total displacement current $I_{D T}$ originates solely from potential changes due to the motion of the ionisation wave front. This hypothesis leads to the following relation:

$$
I_{D T}=i_{e 0} \exp \left((\alpha-\beta) \cdot L_{F}\right)
$$

The electric field $E_{F}$ then becomes the calculated $|E|$ value from our electrostatic measurements (section 3.3), leading to the equivalent expression of the velocity related to the measured lamp parameters:

$$
v_{F}=\frac{I_{D T}}{|E| \cdot L_{F} \cdot C_{l}}
$$

One unknown in (38) is the characteristic length $L_{F}$ of the wave front although it can be inferred from the electrostatic potential measurements. In the present case this parameter is fitted for each amplitude level of $V_{A}$ so the model results match best the experimental data (figure 37). The validation test of the model from electrostatic data then reveals that results are relatively consistent both in absolute values and in trends with the measured pre-breakdown wave velocity. The experimental data points presented in figure 37 are calculated from the raw position data of the E field peak of the wave front. The differences which are observed arise from the initial hypothesis which is not completely verified in our situation since the pre-breakdown wave of negative polarity presents a potential gradient in its wake which evolves over time (figure 10, section 3.1), and thus contributes to the measured $I_{D T}$ value.

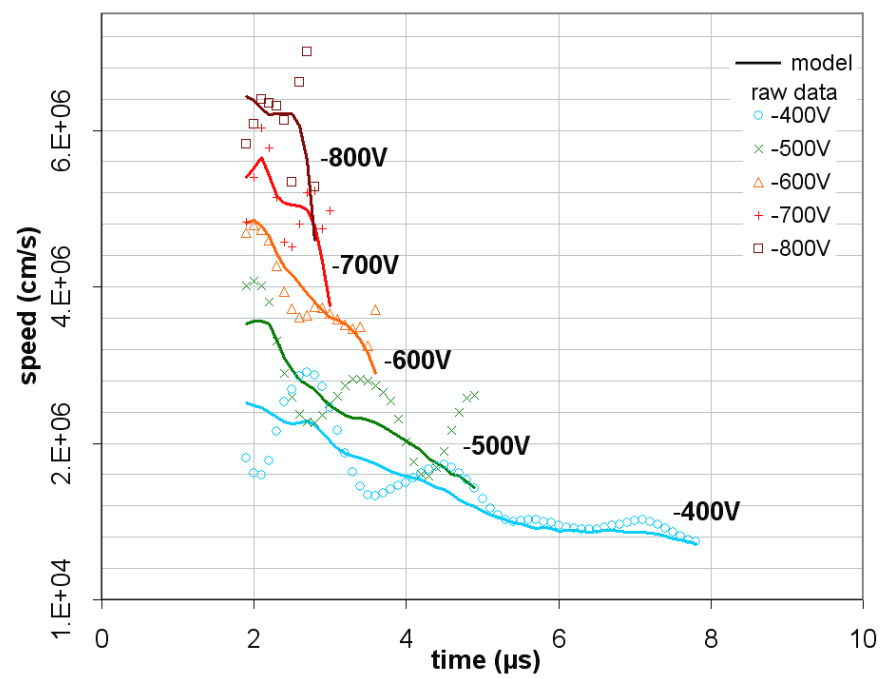

Figure 37: Experimental and model results of the velocity of the pre-breakdown wave front as a function of time and of the voltage applied to the lamp.

A comparison is done between the fitted values of $L_{F}$ and the measured averaged full width at half maximum $\left\langle L_{W E}\right\rangle$ of the axial $\mathrm{E}$ field profile of the pre-breakdown wave from electrostatic measurements. These data are given in table 3 as a function of the applied voltage amplitude $V_{A}$, along with the time range $\Delta t$ when $\left\langle L_{W E}\right\rangle$ was computed and the ratio between this averaged width and $L_{F}$. The results show a linear proportionality between both characteristic lengths, with $L_{F}$ being 6 to $7 \%$ 
narrower than $\left\langle L_{W E}\right\rangle$. This nevertheless indicates that the model gives coherent results and can thus be used in parallel with the electrostatic probe measurements to determine the current fluxes inside the wave front region.

Table 3: Data of the fitted $L_{F}$ values of the wave front length, experimental data of the averaged width $\left\langle L_{W E}\right\rangle$ of the E field profile of the pre-breakdown wave and ratio of these parameters as a function of the applied voltage.

\begin{tabular}{|c|c|c|c|c|}
\hline $\boldsymbol{V}_{\boldsymbol{A}}(\mathbf{V})$ & $\boldsymbol{L}_{\boldsymbol{F}}(\mathbf{c m})$ & $\left\langle\boldsymbol{L}_{W E}\right\rangle(\mathbf{c m})$ & $\boldsymbol{\Delta t}(\boldsymbol{\mu s})$ & $\left\langle\boldsymbol{L}_{W E}\right\rangle / \boldsymbol{L}_{\boldsymbol{F}}$ \\
\hline-400 & 4.2 & 4.5 & $2.1-7.8$ & 1.07 \\
\hline-500 & 5.0 & 5.3 & $2.1-4.9$ & 1.06 \\
-600 & 5.4 & 5.7 & $2.1-4.1$ & 1.06 \\
-700 & 5.6 & 5.9 & $2.3-3.3$ & 1.06 \\
-800 & 6.1 & 6.5 & $2.1-2.9$ & 1.07 \\
\hline
\end{tabular}

The nature of the electron current source in the pre-breakdown wave depends on the polarity of the voltage applied to the active electrode. In the case of ignitions at negative potentials the electron flux emerges from the thermal emission of the active electrode (which is a cathode) and flows through the wake to feed the wave front via the wave boundary. In this case the electron flux $\Gamma_{e w i}$ at the wake inlet is the flux thermally emitted by the active electrode. The flux equality $\Gamma_{e w o}=\Gamma_{e 0}$ then results at the wave boundary, between the wake and the wave front. The end of the electron avalanche in the wave front, where the electron density is $n_{e a}$, thus faces the uncharged and neutral region of the lamp. The wave front model of figure 36 therefore becomes that shown in figure 38 .

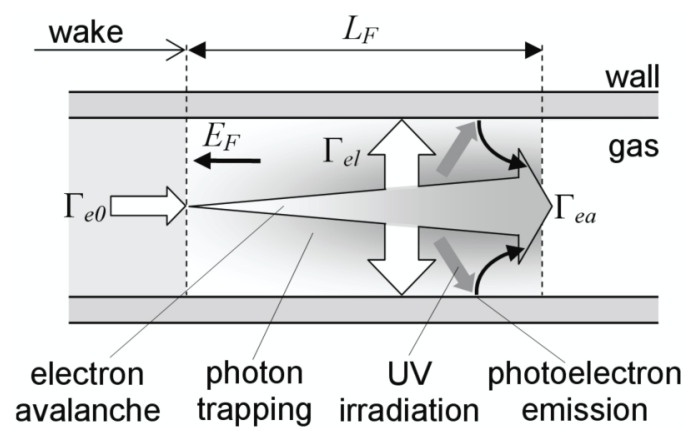

Figure 38: Model of the wave front region of a negative pre-breakdown wave where the primary electrons feeding the avalanche originate for the ionised gas in the wake of the ionisation wave.

The electron avalanche in the wave front region starts at the wave boundary and feeds the wall with radially-diffused electrons. Since the collision between argon atoms and the electrons drifting in the electric field $E_{F}$ also causes the production of UV photons, a photoelectric effect at the wall is then expected to provide with some extra electrons. However, this source does not change the net growth of the avalanche because any emitted photoelectron leaves a local positive charge at the wall [47] which causes the diffusion of an extra electron from the avalanche. It results that any contribution by photoelectric effect is therefore compensated by an enhancement of the radial diffusion of electrons.

The charge diffusion from the avalanche to the wall leads to the local electrostatic charging of the lamp up to the potential $V_{F}$. This process is responsible for the displacement of the axial potential gradient which drives the forward motion of the wave. The propagation of the pre-breakdown wave is therefore affected by the balance between ionization and charge loss to the wall in the wave front, and 
this influence in the wave velocity is the exponential term (i.e. the charge growth factor) in (32). There are three possible cases of figure for the actual balance:

$1 / \alpha<\beta$ - the loss exceeds the gain and the charge growth factor is below unity. The input electron flux $\Gamma_{e 0}$ is mostly directed toward the wall for its charging without contributing to the electron avalanche, resulting in $\Gamma_{e a}<\Gamma_{e 0}$ at the end of the avalanche.

$2 / \alpha \beta \beta$ - the gain exceeds the loss and the charge growth factor is above unity. There is an exponential growth of the electron avalanche leading to $\Gamma_{e a}>\Gamma_{e 0}$.

$3 / \alpha=\beta$ - the gain equals the loss and the charge growth factor is unity. The multiplication of charges in the avalanche replaces exactly any electrons diffused toward the wall, therefore ensuring that $\Gamma_{e a}=\Gamma_{e 0}$ at the end of the avalanche.

Case 1 is not expected to occur in the wave front of pre-breakdown waves which propagate during successful ignitions because this situation (i.e. sub-unity charge growth factor) would lead to a wave extinction within a very short time and distance. The exponential decrease of the electron flux $\Gamma_{e a}$ available for the next avalanche leads to a fast increase of $R_{F}(28)$ which then decreases further the flux $\Gamma_{e 0}$ fed to the wave front. This then results in an exponential decrease of the wave front velocity toward zero, a trend which is not observed in the experimental data points of figure 37.

In the opposite situation of a charge growth factor above unity (case 2) the resulting exponential electron growth in the wave front leads to a wave which propagates autonomously without the need for a current feed from the active electrode. Such runaway avalanche would feature several characteristics which are not observed in our experiments, such as an increasing light intensity and velocity over time and space. Moreover, the electrostatic probe measurements reveal the formation of a cathode sheath in front of the active electrode at the start of the pre-breakdown wave propagation (figure 11, section 3.1), which is evidence of a current flow originating from the electrode. The last elements which rule out case 2 are from the measurements of $I_{D T}$ and of $Q_{T}$ (section 3.4) which clearly indicate the presence of an electron current flowing in the lamp from the active electrode, responsible for continuously charging the lamp during the wave propagation. Since the ionisation occurring in the avalanche produce charges by pair of opposite polarity, its contribution to the total lamp net charge is therefore null and no net lamp displacement current should be associated with a runaway electron avalanche.

It results from these observations that the gain must compensate exactly for the loss in the wave front of the negative pre-breakdown waves investigated in this work. This condition of stability is that the net charge amplification in the wave front is null, i.e. $\Gamma_{e 0}=\Gamma_{e a}$, corresponding to an electron multiplication which only serves the purpose of replacing any charge lost to the wall, eventually resulting in the gradual ionisation of the gas - a consequence of this process rather than a cause. This condition is translated from (27) into:

$$
\exp \left((\alpha-\beta) \cdot L_{F}\right)=1
$$

Relation (39) naturally leads to $\alpha=\beta$ (i.e. case 3 ) and is a logical consequence of the fact that the ionization in the avalanche does not contribute to the net charging of the lamp. This leads to the fundamental hypothesis underlying (37) being in fact $I_{D T}=i_{e O}$.

During the wave propagation the E field strength in the wave front automatically sets itself to a value of $E_{F}=E_{F 0}$ such that (39) is verified. This setting of $E_{F}$ occurs as a consequence of a wave front length $L_{F}$ which adapts itself to the amplitude of the wave boundary potential $V_{F}$ so the wave front reaches a stable avalanche regime. The corresponding wave front length $L_{F 0}$ leading to $\alpha=\beta$ is a stable 
point because any deviation from it leads to an avalanche regime which drives this characteristic length back toward $L_{F 0}$. For instance if $L_{F}$ becomes longer than $L_{F 0}$ then $\mathrm{E}_{\mathrm{F}}<\mathrm{E}_{\mathrm{FO}}$ and the loss exceeds the gain resulting in a quenched electron avalanche (case 1). This leads to a shorter potential gradient which will in turn decrease $L_{F}$ toward $L_{F 0}$ and increase $E_{F}$ back toward $E_{F 0}$. Likewise, any decrease of $L_{F}$ below $L_{F O}$ will increase the wave front $\mathrm{E}$ field strength, resulting in a runaway electron avalanche ( $\alpha \beta \beta$, case 2) which is bound to spread the potential gradient, thus increasing $L_{F}$ back toward $L_{F 0}$. The condition setting the value of $E_{F 0}$ is derived using formula (25) and the expression of $\alpha$ from Ward [50], most suitable for noble gases, leading to the following relation:

$$
\frac{D_{e}}{\mu_{e} E_{F 0}}\left(\frac{R_{J 0}}{R_{L}}\right)^{2}=C \cdot p \cdot \exp \left[-D \cdot \sqrt{\frac{p}{E_{F 0}}}\right]
$$

Where $C$ and $D$ are constants, whose values depend on the nature of the noble gas $\left(29.2 \mathrm{~cm}^{-1} \mathrm{Torr}^{-1}\right.$ and $26.6 \mathrm{Vcm}^{-1 / 2} \mathrm{Torr}^{-1 / 2}$ for argon, respectively), and $p$ is the pressure. The wave front $\mathrm{E}$ field adjusts itself from the potential $V_{F}$ such as $E_{F 0}=V_{F} / L_{F 0}$. The measured electric field amplitude at the peak of the prebreakdown wave (section 3.3) is thus the $E_{F 0}$ value verifying relation (40). Experimental data presented in figure 16 show that $E_{F 0}$ gradually decreases during the wave propagation. This evolution is primarily caused by a decreasing $V_{F}$ amplitude over time (set by (33) and proportional to the wave front potential data of figure 17) which thus requires less diffused electrons to the wall for the charging of the lamp in the wave front section. This trend finds its origin in the fact that the required charge is the local potential times the lamp capacitance. A decrease of $V_{F}$ therefore effectively decreases the $\beta$ constant. It then results that $E_{F}$ must decrease in order to lower the first Townsend coefficient $\alpha$ so as to maintain a breakeven between charge gain and loss, and prevent the wave from entering the runaway regime (i.e. $\alpha>\beta$, case 2).

Although it is observed that the peak E field strength and peak potential amplitude of the wave front decrease with similar trends during the wave propagation (section 3.3), the relative decrease in time is higher for the wave potential. It therefore results that $V_{F}$ decreases relatively faster than $E_{F 0}$, which should lead to a gradual shrinking of the wave front region during the wave propagation. This trend is indeed observed from experimental data; the width at half maximum $L_{W E}$ of the wave front axial E field profile decreases during wave propagation from 5.56 to $2.86 \mathrm{~cm}$ at $-400 \mathrm{~V}$, and from 7.40 to $5.30 \mathrm{~cm}$ at $-800 \mathrm{~V}$ for successful ignitions. For failed ignitions the decrease goes from 4.16 to 2.96 $\mathrm{cm}$ at $-300 \mathrm{~V}$, and the minimum wave front length therefore seems to be approximately $2.9 \mathrm{~cm}$. One exception to these observations is at $-200 \mathrm{~V}$ where $L_{W E}$ actually increases due to the wave extinguishing in the immediate vicinity of the active electrode.

The stability condition (40) met by the electron avalanche has an impact on the expression of the wave velocity derived earlier. It follows from (32) and (39) that this velocity has the corresponding expression:

$$
v_{F}=\frac{i_{e 0}}{E_{F} L_{F} C_{l}}=\frac{i_{e 0}}{V_{F} C_{l}}
$$

Where $V_{F}$ is fixed by the voltage divider formed by $R_{F}$ and $R_{W}$ (33). It is thus inferred from (40) and (41) that the wave is not primarily driven by its wave front $\mathrm{E}$ field (although the strength of $E_{F}$ has indeed an impact on the velocity) or by the resulting local ionisation that occurs there. The primary driver of the wave front is the electron current $i_{e 0}$ which is fed to it from the cathode via the wake. This 
conclusion is also logical since there is no net lamp charging effect from the avalanche alone. The wave front velocity expressed in terms of the initial electron flux $\Gamma_{e w i}$ at the active electrode is then:

$$
v_{F}=\frac{\pi R_{L}^{2} \cdot q_{e} \cdot \Gamma_{e w i} \cdot \exp \left(-\beta_{W} \cdot L_{W}\right)}{E_{F} L_{F} C_{l}}
$$

If the cathode is not driven in the saturation regime (i.e. temperature-defined current [51]) then the expression of the current $i_{e 0}$ at the wave boundary is normally set by the active electrode potential and the total impedance of the wave front and wake such that:

$$
i_{e 0}=\frac{V_{A}-V_{F}}{R_{W}}=\frac{V_{A}}{R_{F}+R_{W}}
$$

Electrostatic potential measurements presented in section 3.1 and the pre-breakdown wave front potential data in section 3.3 show that there is a large potential drop across the wake of the wave. This is due to a relatively large $R_{F}$ value primarily caused by a large loss rate of electrons to the wall (i.e. high $\beta_{F}$ value) which finds its origin from a constantly decreasing wave boundary potential $V_{F}$. This retroaction together with an increasing length of the wake causes a gradual increase of the $R_{F}$ value over time. This in turn leads to a decrease of the electron current $i_{e o}$ responsible for the decrease of the wave velocity (42) and causes a further increase of $R_{F}$ in time. This is this loop process which causes the observed decrease in time and space of the velocity, of the light emission and of the displacement current associated with the negative pre-breakdown wave.

However, the evolution of the wave velocity is not set solely by the characteristic impedances and the cathode potential as suggested by (43). Expressions (28) and (35) clearly show that the impedances involved in the setting of the electron current $i_{e 0}$ are dependant on this same current. The consequence is that the wave current is in fact set for one part by the cathode sheath in front of the active electrode, and for the other part by the total impedance of the pre-breakdown wave. The current will be set such as the following relation is met:

$$
V_{A}=V_{k}+i_{e 0} \cdot\left(R_{W}+R_{F}\right)
$$

Where $V_{k}$ is the voltage drop across the cathode sheath, which can be calculated by assuming a diode model leading to the Child-Langmuir relation [51] between the cathode current $i_{k}$ and $V_{k}$ such that:

$$
i_{k}=\frac{i_{e 0}}{\exp \left(-\beta_{W} \cdot L_{W}\right)}=p_{k} \cdot V_{k}^{3 / 2}
$$

With the $p_{k}$ constant being the electrode perveance, a function of the cathode geometry only. In the situation of failed ignitions the nature and propagation mechanisms of the primary wave are the same as those outlined earlier for successful ignitions. As the lamp propagates its wave front length $L_{F}$ decreases so as to set $E_{F}=E_{F 0}$ and to meet the stability condition (40) necessary for maintaining a stable electron avalanche. The evolution of the wave speed during this phase is still determined by the current fed to the wave front. However, at one point the wave reaches a minimum wave front length which no longer enables a high enough E field strength to be set. This eventually leads to the failure of the 
stability condition (40) and the electron loss exceeds the gain $(\alpha<\beta$, case 1), resulting in the fast quenching of the wave front and the stopping of the pre-breakdown wave propagation.

The analysis of the negative pre-breakdown wave with this simple model leads to several key observations. On the one hand the time evolution of the wave speed is primarily caused by a decrease of the wave current, set by the electrode potential, the total wave impedance and the cathode sheath voltage drop and the electrode temperature. This current decrease finds its origin in a retroactive cycle between an increasing wake impedance $R_{W}$ and a decreasing wave boundary potential $V_{F}$. During propagation the wave front length $L_{F}$ gradually decreases and sets itself so the electron avalanche remains stable. On the other hand the failure of the pre-breakdown wave at low $V_{A}$ settings is caused by a wave front $\mathrm{E}$ field which reaches a critical value below which the charge loss to the wall exceeds the gain. This threshold is crossed when the wave front length reaches a minimum value below which it cannot decrease further. It then results that the main difference between successful ignitions and failed ones is the relation between the decrease rate of $V_{F}$ over space and the length the wave has to propagate in order to connect to the ground electrode. Successful negative polarity ignitions at a given electrode gap length can in fact fail for longer inter-electrode lengths provided these pre-breakdown waves reach their minimal wave front length before connecting with the ground electrode.

\subsection{Pre-breakdown wave of positive polarity}

The model of the pre-breakdown wave velocity developed in section 5.1 is also tested from data at positive polarity using expression (38). It is found that the fit between model and experimental results is optimal for an average $L_{F}$ value of $4.4 \mathrm{~cm}$, nearly constant for all levels of applied potential $V_{A}$. The model results are consistent both in absolute values and in trends with the measured pre-breakdown wave velocity (figure 39), with less differences being observed than for negative ignitions. The reason for this better agreement with experimental data is the much smaller potential gradient in the wake of positive pre-breakdown waves (figure 22, section 4.1), which leads to the hypothesis behind (37) being more valid in the present situation.

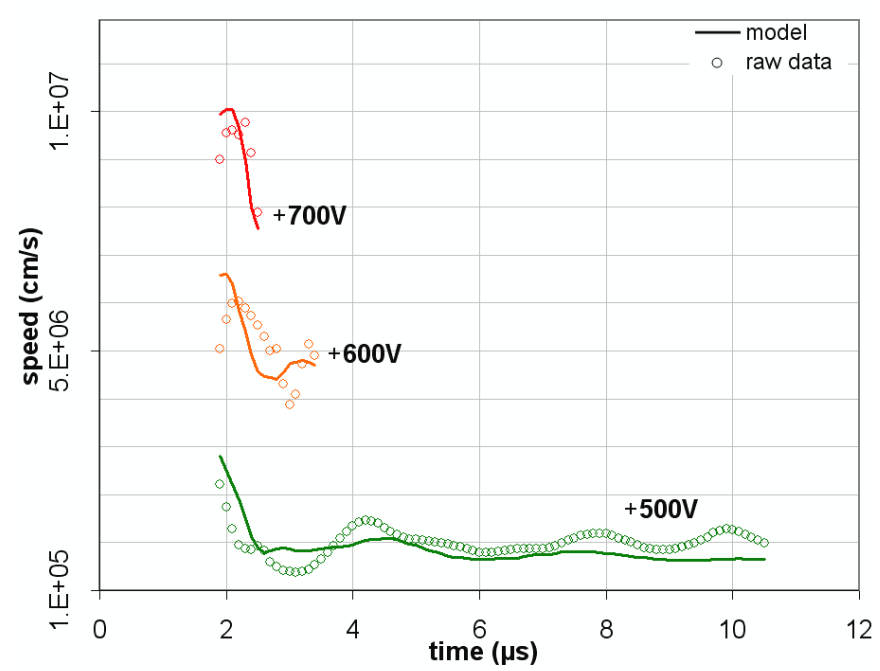

Figure 39: Experimental and model results of the velocity of the pre-breakdown wave front as a function of time and of the voltage applied to the lamp.

A comparison between the fitted $L_{F}$ values and the averaged full width at half maximum $\left\langle L_{W E}\right\rangle$ of the measured axial E field profile of the pre-breakdown wave (table 4) shows a direct proportionality 
between these two parameters. However, the equivalent wave front length $L_{F}$ is about $40 \%$ longer than $\left\langle L_{W E}\right\rangle$, a difference which is much larger than in the case of negative ignitions. The reason for this is most likely a different geometry of the electron avalanche which in the actual lamp is likely to originate from the wall where photo-electric emission occurs (section 4.1). Nevertheless, the fit of the model predicts a nearly constant wave front width which is actually confirmed in the measurements.

Table 4: Data of the fitted $L_{F}$ values of the wave front length, experimental data of the averaged width $\left\langle L_{W E}\right\rangle$ of the $E$ field profile of the pre-breakdown wave and ratio of these parameters as a function of the applied voltage.

\begin{tabular}{|c|c|c|c|c|}
\hline $\boldsymbol{V}_{\boldsymbol{A}}(\mathbf{V})$ & $\boldsymbol{L}_{\boldsymbol{F}}(\mathbf{c m})$ & $\left\langle\boldsymbol{L}_{W E}\right\rangle(\mathbf{c m})$ & $\boldsymbol{\Delta t}(\boldsymbol{\mu s})$ & $\left\langle\boldsymbol{L}_{W E}\right\rangle / \boldsymbol{L}_{\boldsymbol{F}}$ \\
\hline \hline+500 & 4.38 & 2.45 & $2.1-10.5$ & 0.56 \\
+600 & 4.55 & 2.70 & $2.1-3.4$ & 0.59 \\
\hline+700 & 4.45 & 2.70 & $2.1-2.8$ & 0.61 \\
\hline
\end{tabular}

In the case of positive pre-breakdown waves, the source of primary electrons feeding the avalanche in the wave front region is most likely the photo-electron emission from the dielectric wall (section 4.1). The static model of figure 35 must therefore be changed into that of figure 40 to account for the UVcontrolled current source present in the wave front.

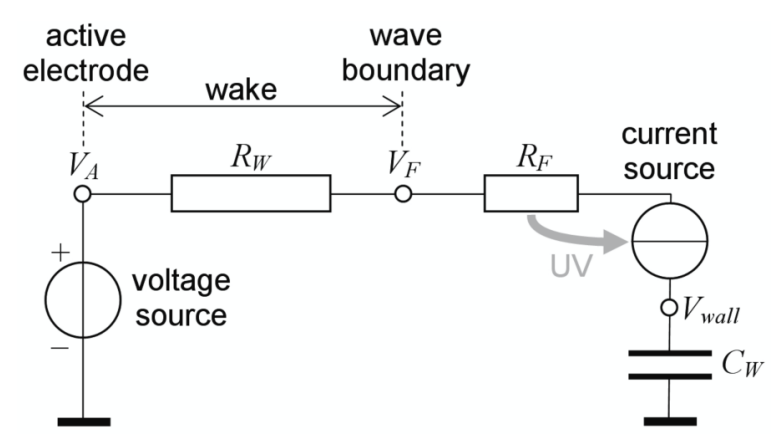

Figure 40: Static electrical and impedance model of a positive polarity pre-breakdown wave where the source of priming current originates from photoelectron emission.

In this new equivalent model the wall potential $V_{\text {wall }}$ still remains zero because of the constant propagation of the wave toward un-charged and neutral lamp sections. The wave front model of figure 36 thus changes into that of figure 41, where the electron avalanche originates at the end of the wave front facing the neutral and un-ionised part of the lamp. The current in the pre-breakdown wave is inverted compared to ignitions at negative potentials; it originates at the wave front and flows via the wave boundary and through the wake to be finally collected by the active electrode which acts as an anode. The electron flux at the wave boundary is thus $\Gamma_{e w i}=\Gamma_{e a}$ and that at the active electrode is therefore $\Gamma_{\text {ewo }}$. 


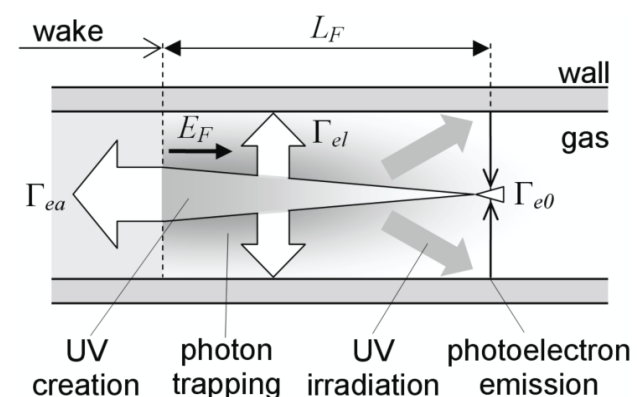

Figure 41: Model of the wave front region of a positive pre-breakdown wave where the primary electrons feeding the avalanche originate from photoelectric emission from the wall.

In the wave front region the primary electrons are emitted via the photoelectric effect as a result of irradiation of the glass wall by UV photons created in the electron avalanche. This emission occurs over the whole surface area enclosed by the wave front but for simplification the photoelectron flux is shown as originating and feeding the electron avalanche from its origin, at right in figure 41 . The rate of photon creation at position $x$ in the avalanche is then:

$$
\left.\frac{d^{2} n_{h v}}{d x \cdot d t}\right|_{x}=\Gamma_{e}(x) \cdot \pi R_{L}^{2} \cdot \frac{K_{e x}}{K_{i}} \cdot \alpha
$$

Where $K_{e x}$ and $K_{i}$ are the reaction rate coefficients of argon atom excitation and of ionisation by electron impact, respectively. Their expressions in terms of electron energy, mass and of energy distribution function is given in equation (12) where for $K_{i}$ the cross section $\sigma$ is that of argon ionisation by electron impact. Calculations with a Boltzmann solver [52,53] show that the ratio between the rate coefficients of argon excitation and of ionisation is best fitted by the following relation:

$$
\frac{K_{i}}{K_{e x}}=A \cdot\left[1-\exp \left(B \cdot\left(E_{0}-E\right)\right)\right]
$$

The three constants in the equation are $A=1.1, B=0.0025 \mathrm{~cm} / \mathrm{V}$ and $E_{0}=35 \mathrm{~V} / \mathrm{cm}$, respectively. Relation (47) is valid for 3 Torr argon in the E field range of 40 to $600 \mathrm{~V} / \mathrm{cm}$, which fully covers the E field strength values encountered during the ignition experiments (section 4.1 and 4.2). The rate of photon creation over the whole wave front length $L_{F}$ follows from (46):

$$
\frac{d n_{h v}}{d t}=\pi R_{L}^{2} \cdot \frac{K_{e x}}{K_{i}} \cdot \int_{0}^{L_{F}} \Gamma_{e 0} \cdot \exp ((\alpha-\beta) \cdot x) \cdot \alpha \cdot d x
$$

Setting the integration constant to zero at $x=0$ (i.e. no photon creation at the origin of the avalanche) yields the following expression:

$$
\frac{d n_{h v}}{d t}=\pi R_{L}^{2} \cdot \frac{K_{e x}}{K_{i}} \cdot \Gamma_{e 0} \cdot \frac{\alpha}{\alpha-\beta} \cdot\left[\exp \left((\alpha-\beta) \cdot L_{F}\right)-1\right]
$$


From this total amount of photons created in the wave front volume only a certain fraction will reach the wall. This fraction is the ratio between the wall surface over the total surface enclosed by the wave front space, giving for the ratio the value $L_{F} /\left(L_{F}+R_{L}\right)$. For each photon reaching the wall only a fraction $\gamma$ (the photoelectric quantum efficiency) will lead to the emission of photoelectrons. The creation rate of photoelectrons is thus expressed from (49) by neglecting the re-absorption losses of UV photons in argon:

$$
\frac{d n_{e p}}{d t}=\pi R_{L}^{2} \cdot \frac{K_{e x}}{K_{i}} \cdot \Gamma_{e 0} \cdot \frac{L_{F}}{L_{F}+R_{L}} \cdot \frac{\alpha \gamma}{\alpha-\beta} \cdot\left[\exp \left((\alpha-\beta) \cdot L_{F}\right)-1\right]
$$

The flux $\Gamma_{e p}$ of photoelectrons through the cross section of the lamp then follows:

$$
\Gamma_{e p}=\frac{K_{e x}}{K_{i}} \cdot \Gamma_{e 0} \cdot \frac{L_{F}}{L_{F}+R_{L}} \cdot \frac{\alpha \gamma}{\alpha-\beta} \cdot\left[\exp \left((\alpha-\beta) \cdot L_{F}\right)-1\right]
$$

Naturally, a stable propagation of the ionisation wave front requires the equality $\Gamma_{e p}=\Gamma_{e 0}$ resulting from a null net evolution of the production rate of photons over time, thus leading to the wave front stability condition:

$$
\frac{K_{e x}}{K_{i}} \cdot \frac{\alpha}{\alpha-\beta} \cdot\left[\exp \left((\alpha-\beta) \cdot L_{F}\right)-1\right]=\frac{L_{F}+R_{L}}{L_{F} \cdot \gamma}
$$

Assuming that the wave front adjusts its length $L_{F}$ so as to reach a critical E field strength enabling condition (52) to be verified, it then results that $L_{F}=V_{F} / E_{F}$ and this leads to the general form of the stability condition:

$$
\frac{V_{F}}{E_{F}} \cdot\left[\frac{K_{e x}}{K_{i}} \cdot \frac{\alpha}{\alpha-\beta} \cdot\left[\exp \left((\alpha-\beta) \cdot L_{F}\right)-1\right]-\frac{1}{\gamma}\right]=\frac{R_{L}}{\gamma}
$$

The left part of (53) is a function of the electric field strength $E_{F}$, while the right side of the equality is a constant. The regime of the electron avalanche will have different consequences on the stability condition. As for the previous section the three different cases are reviewed below:

$1 / \alpha<\beta$ - the loss exceeds the gain and the charge growth factor is below unity. In this case the condition (53) is rewritten as follows:

$$
\frac{V_{F}}{E_{F}} \cdot\left[\frac{K_{e x}}{K_{i}} \cdot \frac{\alpha}{\beta} \cdot\left[1-\exp \left(-\beta \cdot L_{F}\right)\right]-\frac{1}{\gamma}\right]=\frac{R_{L}}{\gamma}
$$

Since the right term of (54) is positive, then the following relation must be verified as a prerequisite to the validity of the stability condition:

$$
\frac{K_{e x}}{K_{i}} \cdot \frac{\alpha}{\beta} \cdot\left[1-\exp \left(-\beta \cdot L_{F}\right)\right]>\frac{1}{\gamma}
$$


The quantum efficiency $\gamma$ of photocathodes designed for photomultiplier tubes ranges from 0.5 to $30 \%$ [51], so it is expected that the $\gamma$ value of the soda lime silicate glass wall of our lamp is much lower than $0.1 \%$, leading to the right term of (55) being higher than 1000 . The ratio $K_{e x} / K_{i}$ as calculated from (47) has a maximum value of 73 in the E field range covered by this fit function, while the ratio $\alpha / \beta$ is below unity and the term between brackets ranges from 0 to 1 . It thus results that (55) is not true and the stability condition (53) is never met when the charge loss to the wall exceeds the gain in the avalanche. In such case the wave front region is bound to extinguish as a result of the breaking of the photoelectron cycle. An electron avalanche maintained in a stable regime in this condition (i.e. $\alpha<\beta$ ) would require a photoelectric quantum efficiency exceeding $1 \%$, which is not realistic in our case.

2/ $\alpha \beta$ - the gain exceeds the loss and the charge growth factor is above unity. In this case the condition (53) evolves into the following relation:

$$
\frac{V_{F}}{E_{F}} \cdot\left[\frac{K_{e x}}{K_{i}} \cdot\left[\exp \left(\alpha \cdot L_{F}\right)-1\right]-\frac{1}{\gamma}\right]=\frac{R_{L}}{\gamma}
$$

Equation (56) is potentially valid for a high enough value of $\alpha \cdot L_{F}$ in the exponential term, which indicates that a critical $\mathrm{E}$ field strength and wave boundary potential level are required for the avalanche to be stable in this regime.

$3 / \alpha=\beta$ - the gain equals the loss and the charge growth factor is unity. Condition (53) is then rewritten in the following form:

$$
\frac{V_{F}}{E_{F}} \cdot\left[\frac{K_{e x}}{K_{i}} \cdot \alpha \cdot L_{F}-\frac{1}{\gamma}\right]=\frac{R_{L}}{\gamma}
$$

Since the first Townsend coefficient ranges from 0.04 to $12 \mathrm{~cm}^{-1}$ in the E field range covered by (46) and the length $L_{F}$ is in the order of a few centimetres (table 4), relation (57) is also potentially valid depending on the value of $\gamma$, and as in case 2 it should require a certain critical $E_{F}$ and $V_{F}$ values for the stability condition to be met.

A further assessment of the validity condition of (56) and (57) is done by calculating the photoelectric quantum efficiency of the glass wall from experimental data at different $V_{A}$ levels and comparing the results to the initial hypothesis (i.e. $\gamma 0.1 \%$ ). The expression of $\gamma$ relative to each avalanche regime is derived using their respective stability condition, yielding the following relations where $E_{F}=E_{F 0}$ for a stable avalanche and photoelectron cycle:

$$
\begin{gathered}
\gamma_{\alpha=\beta}=\left(\frac{V_{F}}{E_{F 0}}+R_{L}\right) \cdot\left(\frac{E_{F 0}}{V_{F}}\right)^{2} \cdot \frac{K_{i}}{K_{e x}} \cdot \alpha^{-1} \\
\gamma_{\alpha>\beta}=\left(1+\frac{E_{F 0} \cdot R_{L}}{V_{F}}\right) \cdot \frac{K_{i}}{K_{e x}} \cdot\left(\exp \left(\alpha \frac{V_{F}}{E_{F 0}}\right)-1\right)^{-1}
\end{gathered}
$$

The tube radius in equations (58) and (59) is known $\left(R_{L}=0.5 \mathrm{~cm}\right), E_{F 0}$ is the measured peak wave field strength and the wave boundary potential is considered to be equal to the active electrode voltage (i.e. 
$V_{F} \approx V_{A}$ ). The last approximation is justified by measurements presented in section 4.1 (i.e. lack of potential gradient in the wake) which logically results in a very low wake impedance value $R_{W}(35)$ since $E_{W}$ is low and $\Gamma_{\text {ewo }}$ is high.

The calculation of $\gamma$ is done from data related to successful ignitions, at the exception of $+700 \mathrm{~V}$ whose pre-breakdown wave finishes its propagation too early and is therefore influenced by the anode sheath formation (section 4.4). At $V_{A}=+500 \mathrm{~V}$ the average $\mathrm{E}$ field strength $E_{F O}$ in the plateau is $178 \mathrm{~V} / \mathrm{cm}$ (section 4.3), which from calculations yields $\gamma=5.0 \%$ for $\alpha=\beta$ and $0.016 \%$ for $\alpha>\beta$. At $+600 \mathrm{~V}$ the value of $E_{F 0}$ is $137 \mathrm{~V} / \mathrm{cm}$ leading to $\gamma=3.7 \%$ for $\alpha=\beta$ and $0.015 \%$ for $\alpha \beta \beta$. It results that condition (57) corresponding to $\alpha=\beta$ requires an unrealistically high $\gamma$ value in order to be valid, while relation (56) is verified for a photoelectron quantum efficiency which is more coherent with the nature of the wall material. Moreover, the results for $\alpha \beta$ are consistent between each others while this is not the case for $\alpha=\beta$. $\gamma$ is expected to be a constant since the resonant radiation responsible for the emission of photoelectron does not vary in wavelength with the nature of the electron avalanche.

The conclusion from this analysis is that the electron avalanche in the wave front of positive prebreakdown waves has to be of the runaway type (i.e $\alpha>\beta$, case 2) for the photoelectron cycle to be stable. This outcome is logical since very few photoelectrons are expected to be emitted from the wall due to a low $\gamma$ value, which then requires a very strong charge multiplication to occur in the avalanche for the lamp section enclosed by the wave front to be charged up to the potential $V_{F}$. Since the net electrostatic charge responsible for the displacement of the potential gradient is positive, it then results that this space charge is primarily caused by free argon ions built-up in the lamp volume from the avalanche process. This situation is the opposite of negative ignitions where the space charge is primarily caused by a negative wall charging resulting from the radial electron diffusion from the avalanche. However, there is still an electron diffusion to the wall in the positive wave front due to the thermal motion of electrons, as pointed out by Langer et al. [20], but its magnitude is much lower than at negative potentials. The main reason for the lower $\beta$ factor is a Faraday cage potential which is negative in respect to that of the wave front, leading to a retarding electric field at the wall which repels the less energetic electrons.

The run-away avalanche encountered in our situation does not lead to the same wave front properties as those outlined in previous section for this regime, namely an autonomous wave whose charge density, light emission and velocity would increase in time and space. At positive polarity the avalanche grows toward the active electrode but is stopped at the wave boundary where the amplified electron current is collected by the low-impedance wake and is conducted away to the anode. However, the positive pre-breakdown wave is nonetheless a self propagating wave as long as the photoelectron cycle in the wave front is stable. Since the E field adjust itself so as to reach this stability, and as the wake impedance is very low in regard to that of the wave front, the propagation of the positive pre-breakdown wave is therefore much less affected by distance than at negative polarity; this is the reason behind the observed regularity of the wave propagation, and the direct consequence is a lamp ignition voltage being also much less affected by the inter-electrode distance. This can be understood further by considering the expression of the wave front velocity (32) and the fact that the priming current $i_{e 0}$ is the photoelectron current feeding the avalanche, set by the stability condition of the photoelectron cycle. However, due to continuity of current in the equivalent circuit of figure 40 (i.e. the Kirchoff current loop), $i_{e 0}$ is also set by the active electrode potential and the total impedance of the ionisation wave such that:

$$
V_{A}=V_{a}+i_{e 0} \cdot \exp \left((\alpha-\beta) \cdot L_{F}\right) \cdot\left(R_{W}+R_{F}\right)
$$


Where $V_{a}$ is the voltage drop across the anode sheath that surrounds the active electrode. In our case $\alpha>\beta$ in the wave front and $\beta_{W}$ is small in the wake, resulting in (60) being rewritten as:

$$
V_{A}=V_{a}+i_{e 0} \cdot \exp \left(\alpha \cdot L_{F}\right) \cdot R_{F}
$$

Which then results in the following expression for the wave velocity:

$$
v_{F}=\frac{V_{A}-V_{a}}{R_{F} E_{F} L_{F} C_{l}}=\frac{1}{R_{F} C_{l}}
$$

Relations (61) and (62) do not bear any relation with the properties of the wake but only with those of the wave front, which is a direct consequence of $R_{W}$ being much lower than $R_{F}$. This, and the stability condition for the photoelectron cycle, lead to a wave front whose properties are independent on the wave propagation (i.e. especially the wave position in the lamp), hence its self sustained-nature.

The requirement that $\alpha L_{F}$ be high enough for relation (56) to be valid then leads to the following condition at $E_{F}=E_{F 0}$ for a stable avalanche where the wave boundary potential $V_{F}$ is close to that of the active electrode:

$$
\frac{V_{F}}{E_{F 0}} \cdot\left[\frac{K_{e x}}{K_{i}} \cdot \exp \left(\alpha \cdot \frac{V_{F}}{E_{F 0}}\right)-\frac{1}{\gamma}\right]=\frac{R_{L}}{\gamma}
$$

With the $R_{L} / \gamma$ constant at right being equal to $3226 \mathrm{~cm}$. The relatively constant velocity and features of the positive pre-breakdown wave shown in sections 4.1 and 4.2 tend to indicate that condition (63) is indeed verified in our experiments at voltages above $+450 \mathrm{~V}$. The measured full width at half maximum $L_{W E}$ of the E field profile of the wave front remains constant within $\pm 0.27 \mathrm{~cm}$ at $+500 \mathrm{~V}$ and within $\pm 0.15 \mathrm{~cm}$ at $+700 \mathrm{~V}$ during the wave propagation. This is a clear evidence that the photonphotoelectron cycle remains stable. Moreover, the nearly constant displacement current after $2.6 \mu \mathrm{s}$ (section 4.1 and 4.4) indicates that the charge growth factor of the avalanche remains also relatively stable.

The numerical resolution of (63) using the expressions (40) and (47) show that the stability condition of the avalanche is no longer verified for any E field strength at a $V_{F}$ value below $398 \mathrm{~V}$. This threshold is indeed observed in the electrostatic potential profile of the pre-breakdown wave at $+450 \mathrm{~V}$ (failed ignition). A potential dip begins to form at around $20 \mu \mathrm{s}$ in the lamp space between 2 and $8 \mathrm{~cm}$ (figure 32, section 4.5). This formation coincides with a wave boundary potential which decreases below $400 \mathrm{~V}$, and the resulting breach of the photoelectron cycle leads to a slow decrease of the wave propagation as less photoelectrons feed the avalanche over time. The observed consequence is a wave front width $L_{W E}$ which continuously decreases from $2.65 \mathrm{~cm}$, near the active electrode, to 0.9 $\mathrm{cm}$ at the end of the wave propagation. The wave front electric field strength then increases over time and still drives the electron avalanche in the run-away regime. This, together with the decreasing wave speed (primarily caused by the decreasing photoelectron flux), lead to the build-up of an electron space charge at the back of the wave front which neutralises the ion volume charge, thus decreasing the potential further there. This in turn decreases the wave boundary potential and in a retroactive cycle it decreases the wave speed further until it reaches zero. Since the photoelectron flux decreases during this propagation, there is consequently a build up of a strong positive space charge in the wave front which forms a virtual anode there. This is momentarily responsible for stabilizing the local electron 
space charge at the back of the wave front, which will then evolve into a durable local wall charge as a result of the radial diffusion of electrons.

There are several key findings concerning pre-breakdown waves at positive polarity resulting for this model analysis. As opposed to processes at negative polarity the propagation of the positive ionisation wave is more constant and stable over time, which is primarily caused by the wave propagation characteristics being set by the wave front properties, defined by the stability of the photoelectron cycle and by the wave boundary potential $V_{F}$. The stability condition of the wave front is in fact defined by that of the photoelectron cycle rather than the balance between charge gain and loss. It results that the wave front $\mathrm{E}$ field adjusts itself so the photoelectron generation remains constant, leading to an electron avalanche driven in the run away mode $(\alpha>\beta)$. This behaviour finds its origin in the fact that the wave front impedance is much higher than that of the wake, therefore rendering the properties of the positive pre-breakdown wave independent of its propagation or position in the lamp, but also making it self-propagating in nature. This intrinsic characteristic should lead to an ignition voltage nearly independent on the inter-electrode distance. The failure of the positive ionisation wave occurs at a wave boundary voltage threshold below which the photoelectron cycle becomes unbalanced and the photoelectron flux gradually decreases in time and space until the wave stops. During this process the wave front electric field $E_{F}$ increases as a consequence of the physical shrinkage of this region, thus maintaining the electron avalanche in the run away regime. This situation ultimately leads to a positive space charge in the wave front and to the build up of electrons in the back of the wave.

\subsection{Static ignition voltage}

One of the most important characteristics of a gas discharge tube is its ignition voltage whose level has to be known for the design of power supplies. There are many ways to define this voltage [29] and in our situation we define it as the level below which the chance of lamp ignition is null and the delay time of ignition becomes infinite.

It is found in our experiments that the requirement for a successful lamp ignition is that the prebreakdown wave reaches the ground electrode. The static breakdown voltage can thus be related to the formative lag time $t_{f}$ of this process, $V_{b d}$ being therefore defined when $t_{f}$ reaches toward infinity. It is also experimentally found that the average pre-breakdown wave velocity $\left\langle v_{f}\right\rangle$ is proportional to the voltage $V_{A}$ applied to the lamp following the expression:

$$
<v_{f}>=K \cdot\left(V-V_{b d}\right)
$$

Where $K$ is a constant which represents the impact of the overvoltage $\left(V-V_{b d}\right)$ on the wave velocity and depends on the polarity and the type of lamp investigated. The formative lag time $t_{f}$ can be expressed in terms of formula (64) and of the inter-electrode distance $L$ :

$$
t_{f}=\frac{L}{<v_{f}>}=\frac{L}{K \cdot\left(V-V_{b d}\right)}
$$

Expression (65) is thus fitted to the measured $t_{f}$ values for the different applied voltages (see data of table 5), and $V_{b d}$ is found where the function reaches toward infinity (figure 42). The observation that the lamp ignition voltage is higher at positive polarity $(+455 \mathrm{~V})$ than at negative potentials $(-300 \mathrm{~V})$ is a consequence for one part of the different stability conditions required for the sustained propagation of the wave front, and for another part of the influence of the developing wave wake on the 
propagation of the wave front. The stability condition at positive polarity sets the requirements in terms of the photoelectron cycle, which in turn requires a higher wave front $\mathrm{E}$ field strength $E_{F}$ and wave boundary potential $V_{F}$ than at negative polarity, where the condition is only the charge loss being equal to the gain in the electron avalanche. This difference alone could explain the higher breakdown voltage at positive potential, but the evolution of the wave characteristics during propagation is also a very important factor determining $V_{b d}$.

Table 5: Measured formative lag time $t_{f}$ as a function of the applied voltage level $V_{A}$. The values of the fitting parameters of equation (65) are also given.

\begin{tabular}{|c|c|c|c|}
\hline \multicolumn{2}{|c|}{ Negative ignition } & \multicolumn{2}{c|}{ Positive ignition } \\
$V_{A}(\mathrm{~V})$ & $t_{f}(\mu \mathrm{s})$ & $V_{A}(\mathrm{~V})$ & $t_{f}(\mu \mathrm{s})$ \\
\hline \hline-400 & $10.0 \pm 0.1$ & - & - \\
\hline-500 & $5.7 \pm 0.1$ & +500 & $12.4 \pm 0.1$ \\
-600 & $4.2 \pm 0.1$ & +600 & $3.8 \pm 0.1$ \\
-700 & $3.4 \pm 0.1$ & +700 & $2.7 \pm 0.1$ \\
\hline-800 & $3.0 \pm 0.1$ & - & - \\
\hline$K\left(\mathrm{mV}^{-1} \mathrm{~s}^{-1}\right)=$ & 132 & $K\left(\mathrm{mV}^{-1} \mathrm{~s}^{-1}\right)=$ & 242 \\
\hline \multicolumn{2}{|c|}{$V_{b d}(\mathrm{~V})=$} & 300 & $V_{b d}(\mathrm{~V})=$ \\
\hline
\end{tabular}

As pointed out in previous sections the minimum voltage required for the full propagation of the negative ionisation wave is linked to the decrease of its wave boundary potential during propagation, and a shorter electrode gap will then lead to a lower ignition voltage. In comparison, the processes at positive potentials are independent on the wave propagation and are therefore much less affected by the length of the lamp. It therefore results that longer lamp lengths should increase the negative ignition voltage up to a level equal or higher than that at positive polarity, thus offsetting the effects due to differences in terms of wave front stability condition.

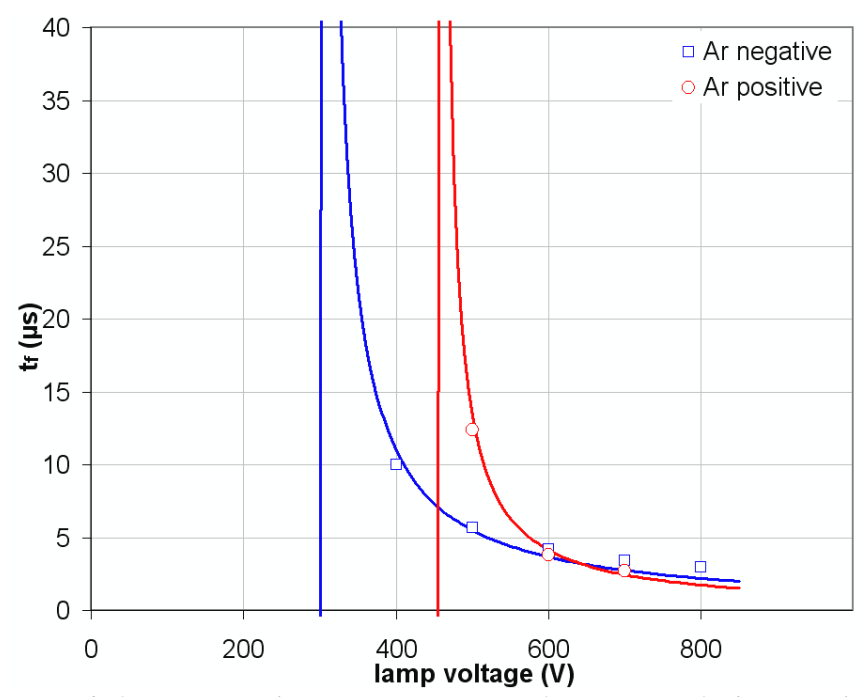

Figure 42: Measured formative lag time $t_{f}$ as a function of the applied negative and positive voltage levels to the 3 Torr argon lamp. The solid lines are the fitting functions used for the determination of the static ignition voltages. 
The higher sensibility (i.e. higher $K$ factor) of positive ignition to the over voltage $V-V_{b d}$ is due to the electron avalanche in the wave front being always in the run away mode $(\alpha>\beta)$ while in the wave front of negative pre-breakdown wave there is a maintained breakeven between charge gain and loss $(\alpha=\beta)$. It results that the positive wave front will experience a larger increase of charge multiplication at higher applied voltages, finally leading to faster wave propagations and therefore to shorter formative time lags.

It was remarked in sections 3 and 4 that there are three requirements for the pre-breakdown wave to complete its propagation and ensure a successful lamp ignition:

- the applied voltage $V_{A}$ must be higher than the static ignition voltage $V_{b d}$ of the lamp

- the applied pulse duration $\Delta t_{p}$ must be long enough to let the ionization wave complete its course

- a minimum amount of charge $Q_{T m}$ must be supplied to the lamp

It is therefore interesting to note that formula (65) establishes the first relation between the applied voltage and the pulse duration needed for a successful lamp ignition:

$$
\Delta t_{p} \cdot\left(\left|V_{A}\right|-\left|V_{b d}\right|\right)=\frac{L}{K}
$$

Relation (66) is only valid for $\left|V_{A}\right|>\left|V_{b d}\right|$ and the constant $K$ depends on the polarity of the applied voltage and on the nature of the lamp (gas fill, pressure ...). Next, the relation between the minimum amount of charge and the applied voltage level is derived from the analysis done in section 3.4 and 4.4 relative to the capacitive behaviour of the lamp during ignition. Formulas (15) and (16) lead to the following expression for this relation:

$$
Q_{T m}=V_{A} \cdot C_{T} \cdot\left(\kappa \cdot V_{A}+\zeta\right)
$$

Expression (67) is valid for $\left|V_{A}\right|>\left|V_{b d}\right|$ and $\kappa$ and $\zeta$ are constants which depend on the polarity of $V_{A}$ and on the nature of the lamp. Equations (66) and (67) are then the fundamental relations between the three conditions that are to be met in order to ensure the successful ignition of linear lamps with unipolar voltage pulses.

\section{Conclusion}

We investigated the phenomena of electrical breakdown which occur inside a low pressure argon discharge lamp upon the application of positive and negative unipolar voltage pulses in the subkilovolt range. This study was performed with a fast intensified CCD camera and a novel electrostatic probe. The latter was specifically developed so as to enable the measurements of the lamp electrostatic potential in time and space without inducing any notable influences on the ignition processes. We described the design principles of this instrument and detailed the calculation procedures which were used for the computation of parameters, such as the electric field amplitudes, the displacement current and the net charge, which were displayed in space-time diagrams for cross comparisons enabling a full analysis of the observed phenomena. Moreover, the guidelines were given for the proper operation of the lamp so as to minimize the impact of external factors on breakdown phenomena.

Experiments show that three distinct phases exist during successful ignitions: Upon the application of voltage a first ionization wave starts from the active electrode and propagates in the neutral gas toward the opposite electrode. This wave is associated with a local front of high E field strength whose 
profile follows that of the light emission pattern. This E field is essentially axial in nature in the wave front region, and it causes a local Townsend-like ionisation of the argon gas which leads to the electrostatic charging of the lamp. This observation is corroborated by electrostatic charge and displacement current measurements. An offset between the peak of light emission and that of E field strength is observed and is attributed to the spatial growth of the electron density in the wave front. The observed wave velocity typically ranges from $10^{6}$ to $10^{7} \mathrm{~cm} / \mathrm{s}$ and its averaged value is found to be proportional to the applied voltage. It is furthermore observed that the first wave does not end its propagation by physically reaching the opposite (ground) electrode, but reaches a point in the lamp where the E field strength developed in the space between the wave front and the ground electrode will be sufficiently high to cause a global ionization of this volume, effectively bridging the gap and closing the electrical circuit.

In the next phase, the volume ionisation leading to the wave-electrode gap bridging initiates a second wave (the return stroke) which propagates from the ground electrode back toward the active electrode. This propagation leads to a partial discharging of the lamp as a consequence of a redistribution of the electrostatic charge and of the potential across the lamp. The wave is therefore associated with a reversed displacement current whose profile and evolution matches that of the optical emission. This wave propagates faster than the pre-breakdown waves, with a velocity that ranges from $10^{6}$ to $3.9 \cdot 10^{7} \mathrm{~cm} / \mathrm{s}$. It is found that the return stroke draws a homogeneous plasma column in its wake which eventually connects both electrodes at the end of the wave propagation. Both electrode sheaths are formed and the common features of a glow discharge are observed once the return stroke has reached the active electrode. The third phase is an increase of the light intensity of the plasma column until the lamp reaches a steady state operation set by an external impedance connected to the active electrode.

There are many differences observed in the breakdown phenomena associated with potentials of positive and negative polarity. The negative pre-breakdown waves have a velocity which decreases in time and space while those at positive potentials presents more constant features and velocity in time. This difference is also observed in the profiles of the E field amplitude and of the displacement current. Moreover, the negative pre-breakdown wave presents a potential gradient in its wake which is caused by an incomplete charging of the lamp while at positive polarity the lamp section behind the wave front is charged to a potential close to that of the active electrode, leading to a negligible gradient. It is also found that the source of primary electrons feeding the ionisation wave front at positive polarity is most likely via a photoelectric effect at the dielectric wall which is irradiated by UV photons. There are differences observed for the return stroke as well, with that at negative polarity propagating at much lower velocity than at positive potentials. The electric field strength in the positive return stroke wave front is found to be lower than at negative polarity.

Failed ignitions are found to be caused by a pre-breakdown wave which does not reach the opposite electrode. This observation and the measurements of the lamp net electrostatic charge led to the conclusion that there are three requirements for a successful lamp ignition: the applied voltage must be equal of higher than the static ignition voltage, the time duration of the applied voltage pulse must be long enough to let the pre-breakdown wave complete its propagation in the lamp, and finally there is a minimum amount of charge that needs to be supplied to the lamp for its electrostatic charging. Failing to meet any one of these requirements leads to a first wave which starts its propagation but extinguishes in the lamp before reaching the ground electrode, resulting in the development of a permanent charge memory effect which will negatively affect the next ignition attempts at a given polarity. 
An analytical model of the ionisation waves was derived and used in conjunction with the experimental data in order to better understand the influence and evolution of different internal parameters. The outcome of this analysis is that the electric field strength in the wave front region adjusts itself so as to meet a stability condition which is different depending on the polarity of the applied voltage. At negative potentials this condition is a charge creation rate in the electron avalanche which balances exactly the charge loss rate to the wall. The primary driver of the wave propagation is the electron current which is fed to the wave front via the wake of the wave. The negative prebreakdown wave has therefore a velocity which is set by the total wave impedance (wake and wave front) and the voltage drop across the cathode sheath. Since the length of the wake increases during the forward motion of the wave, its total impedances gradually increases, resulting in the gradual decrease of the current fed to the wave front. This evolution turns out to be responsible for the observed gradual decrease of the wave velocity over time.

At positive polarity the stability condition sets a balance in the production of photoelectron at the wall resulting from UV irradiation from the electron avalanche. This condition leads to a higher wave front $\mathrm{E}$ field amplitude than at negative polarity, which results in an run away electron avalanche where the charge gain exceeds the loss to the wall. The direct consequence is a full electrostatic charging of the wake of the positive pre-breakdown wave, leading to a much lower ohmic impedance of the wake than of the wave front. It results that the wave propagation characteristics are primarily set by the wave front properties, which makes the wave self-propagating in nature and nearly independent on the wave front position in the lamp. The photo-electron cycle responsible for this wave propagation is primarily driven by the wave front $\mathrm{E}$ field. The resulting self-sustained cycle is maintained as long as the electron current emerging from the photoelectron emission and the avalanche process is collected by the anode via the wake of the wave.

In the final section the lamp the static ignition voltages were calculated from a formative lag time analysis, and shows that the lamp requires a lower voltage to ignite at negative potentials. This is found to be primarily caused by a short inter-electrode distance and it is expected that the case would be the opposite for a longer lamp length due to the positive ignition voltage being nearly independent on the inter-electrode distance. Moreover it was shown that the decrease of the formative lag time of positive pre-breakdown wave over the applied voltage is faster than at negative potentials. This is caused by an electron avalanche in the positive pre-breakdown wave front which has a much larger charge growth factor. Finally, the relations between the three ignition requirements are derived from this analysis.

In this study it was demonstrated that a properly designed experimental set-up can provide with several time- and space-resolved plasma-related parameters, with were retrieved with a minimal level of lamp perturbation. It was also shown that a coherent analytical model could be derived with the knowledge gained from measurements. Experimental data combined with this model thus enabled a deeper understanding of the macroscopic processes that occur in the argon discharge lamp during the early phases of its unipolar ignition. A better understanding of fluorescent lamp ignition can therefore be gained by applying the same diagnostics and methods to discharge tubes filled with an argonmercury mixture. Moreover, the influence of the inner fluorescent coating on the fundamental breakdown processes could be understood further. Finally, the analytical model could be developed further so as to include a description of the return stroke and provide some insight into ignition processes at alternating potentials.

\section{Acknowledgment}

This work was supported by Philips Lighting, Eindhoven, the Netherlands. The authors would like to thank Fred Westerterp, from Philips Lighting, for making and processing the lamp. The authors 
express their gratitude also to Huub van den Nieuwenhuizen, from Philips Lighting, and to Wouter Brok from the Eindhoven University of Technology for sharing their expertise and for the highly interesting and fruitful discussions in the field of ignition physics.

\section{References}

[1] Anders A 2003 Tracking down the origin of arc plasma science - II. Early continuous discharges IEEE Trans. Plasma Sci. 31:4 1060-1069

[2] Scott R 1914 The evolution of the lamp Trans. Ill. Eng. Soc. 9:2 138-163

[3] Claude G 1913 Neon tube lighting Trans. Ill. Eng. Soc. 8:7 371-378

[4] Buttolf L J 1920 The Cooper-Hewitt mercury vapor lamp; Part I. Theory and applications Gen. Elec. Rev. 23:9 741-751

[5]Patterson C C 1933 Luminous discharge tube lighting G.E.C. Journal 4:1 46-59

[6] Kelting R C and Buttolf L J 1938 Improvements in the low pressure mercury vapor lamp Trans. Ill. Eng. Soc. 33:7 643-655

[7] Forsythe W E and Adams E Q 1948 Fluorescent and other gaseous discharge lamps (NewYork:Murray-Hill Books, inc.)

[8] Inman G E 1939 Characteristics of fluorescent lamps Trans. Ill. Eng. Soc. 34:1 65-86

[9] Bouwknegt A 1982 Compact fluorescent lamps J. Ill. Eng. Soc. 11:4 204-212

[10] Devonshire R 2008 The competitive technology environment for LED lighting J. Light \& Vis. Env. 32:3 275-287

[11] Coaton J R and Marsden A M 1997 Lamps and lighting 4th edn (London: Arnold)

[12] Townsend J S 1914 Electricity in gases (Oxford:Oxford University Press)

[13] Lisovskiy V A, Yakovin S D and Yegorenkov V D 2000 Low-pressure gas breakdown in uniform dc field J. of App. Phys.:D 33 2722-2730

[14] Waymouth J 1971 Electric discharge lamps (Cambridge:MIT press)

[15] Nedospasov A V and Novik A E 1961 The speed of propagation of the ionisation front in breakdown in long discharge tubes Soviet Phys.-Tech. Phys. 5 1261-1267

[16] Horstman R E and Oude-Lansink F M 1988 The starting process in long discharge tubes $J$. of Phys. D: Appl. Phys. 21 1130-1136

[17] Popa G, Ohe K and Dumitraşcu N 1989 On the detection of both the ionization waves and the socalled echo phenomenon by Langmuir and capacitive probes J. of Appl. Phys. 22 1327-1332

[18] Dengra A, Hernandez M A and Colomer V 1987 A study of ionization front velocity in dc pulsed plasmas Contr. to Plasma Phys. 27 283-292

[19] Ballesteros J, Hernández H, Dengra A and Colomer V 1991 An experimental and theoretical study of the creation plasma process in dc pulsed discharges Cont. to Plasma Phys. 30 595-604

[20] Langer R, Garner R, Hilscher A, Tidecks R and Horn S 2008 Propagation of ionization waves during ignition of fluorescent lamps J. Phys. D: Appl. Phys. 41/144011 (9pp.)

[21] von Busch H, Roozekrans C and Lefki K 2001 Investigation of the ignition process in long narrow cold-cathode neon lamps Proc. $9^{\text {th }}$ Symp. Sci. Tech. Light Sources 149-150

[22] Brok W J M, van Dijk J, Bowden M D, van der Mullen J J A M and Kroesen G M W 2003 A model study of propagation of the first ionization wave during breakdown in a straight tube containing argon J. of Phys. D: Appl. Phys. 36 1967-1979

[23] Brok W J M 2005 Modelling of transient phenomena in gas discharges (PhD Thesis - Eindhoven University of Technology)

[24] Burgmans A L J and Smeets A H M 1983 The ionization coefficient in Ar-Hg mixture J. of Phys. D: Appl. Phys. 16 755-762 
[25] Dushman S and Lafferty J M 1962 Scientific foundation of vacuum technique (New-York: John Wiley \& Sons)

[26] Daelman C and Hilscher A 2008 Why is a 100h burn-in period necessary Lighting Magazine 28:4 34.

[27] Dorleijn J and Goud L 2002 Standardization of the static resistances of fluorescent lamp cathodes and new data for pre-heating Proc. IEEE-IAS Conf. 665-672

[28] Myojo M and Fukumasa O 2004 Some aspect of the interface between fluorescent lamps and electronic gears Proc. $10^{\text {th }}$ Symp. Sci. Tech. Light Sources 25-34

[29] Pejović M M, Ristić G S and Karamarković J P 2002 Electrical breakdown in low pressure gases J. of Phys. D: Appl. Phys. 35 R91-R103

[30] Weston G F 1968 Cold cathode glow discharge tubes (London:Iliffe books)

[31] Thayer R N and Hinman D D 1945 Requirements for reliable instant-starting fluorescent lamps Illum. Eng. 40 640-662

[32] McFarland R H and Sargent T C 1950 Humidity effect on instant starting of fluorescent lamps Illum. Eng. 45 423-428

[33] McFarland R H 1951 The cause of the humidity effect in fluorescent lamps Illum. Eng. 46 345349

[34] Acton J R and Swift J D 1963 Cold cathode discharge tubes (London: Heywood \& Company Ltd)

[35] Groth R and Kauer E 1967 Thermal insulation of sodium lamps Philips Tech. Rev. 26 105-111

[36] van Boort H J J and Groth R 1968 Low-pressure sodium lamps with indium oxide filter Philips Tech. Rev. 26 17-18

[37] Hummel R E 1992 Electronic properties of materials (New-York: Springer)

[38] Hammer E E 1995 Cathode fall voltage relationship with fluorescent lamps J. of the I.E.S. 24 116122

[39] Nachtrieb R, Khan F and Waymouth J F 2004 Improved method for making capacitive measurements of cathode fall in fluorescent lamps Proc. $10^{\text {th }}$ Symp. Sci. Tech. Light Sources 285-286

[40] Jansson P A 1997 Deconvolution of images and spectra (New-York: Academic Press)

[41] Marchetti A A and Mignerey A C 1992 Deconvolution of mass spectra Nucl. Instrum. Methods A324 288-296

[42] Savitzky A and Golay J E 1964 Smoothing and differentiation of data by simplified least square procedures Anal. Chem. 36 1627-1639

[43] Stiener J, Termonoia Y and Deltour J 1972 Comments on smoothing and differentiation of data by simplified least square procedure Anal. Chem. 44 1906-1909

[44] Sigeneger F, Winkler R and Robson R E 2003 What really happens with the electron gas in the famous Frank-Hertz experiment? Contrib. Plasma Phys. 43 178-197

[45] Llewellyn-Jones F 1957 Ionization and breakdown in gases (London: Methuen \& Co Ltd)

[46] Ellis H W, Pai R Y, McDaniel E W, Mason E A and Viehland L A 1976 Transport properties of gaseous ions over a wide energy range At. Data Nucl. Data Tables 17:3 177-210

[47] Cobine J D 1958 Gaseous conductors - theory and applications (New York: Dover)

[48] Elenbaas W 1972 Light sources (London: MacMillan Press Ltd)

[49] Reeth Roth J 1995 Industrial plasma engineering, V.1 (Bristol: IOP)

[50] Ward A L 1958 Effect of space charge in cold cathode gas discharge Phys. Rev. 112 1852-1857

[51] Sedlaček M 1996 Electron physics of vacuum and gaseous devices (New York: John Wiley \& Sons, Inc.)

[52] Hagelaar G 2008 Bolsig + version 1.1 Laplace-CNRS Université Paul Sabatier, France 
[53] Hagelaar G J M and Pitchford L C 2005 Solving the Boltzmann equation to obtain electron transport coefficients and rate coefficients for fluid models Plasma Sources Sci. Technol. 14 722-733 